\title{
NUTRITION AND HEALTH-RELATED QUALITY OF LIFE IN OLDER ADULTS IN THE US, NHANES 2009-2012
}

\author{
A Thesis \\ presented to \\ the Faculty of California Polytechnic State University, \\ San Luis Obispo
}

In Partial Fulfillment

of the Requirements for the Degree

Master of Science in Agriculture,

With a Specialization in Food Science and Nutrition 
(C)2016

Jessica Anne Hews

ALL RIGHTS RESERVED 
TITLE: $\quad$ Nutrition and Health-Related Quality of Life in Older Adults in the US, NHANES 2009-2012

AUTHOR: Jessica Anne Hews

DATE SUBMITTED: April 2016

COMMITTEE CHAIR: Kari Pilolla, Ph.D., R.D.

Assistant Professor of Food Science and

Nutrition

COMMITTEE MEMBER: Louise Berner, Ph.D.

Professor of Food Science and Nutrition

COMMITTEE MEMBER: Ulric Lund, Ph.D.

Professor of Statistics

COMMITTEE MEMBER: $\quad$ Lisa Nicholson, Ph.D., R.D.

Professor of Food Science and Nutrition 


\begin{abstract}
Nutrition and Health-Related Quality of Life in Older Adults in the US, NHANES 20092012

Jessica Anne Hews
\end{abstract}

Few studies have investigated the relationship between diet quality and healthrelated quality of life ( $\mathrm{HRQOL})$, especially in the older adult population that is most at risk for poor diet and health. Self-perceived HRQOL is a more powerful predictor of morbidity and mortality than objective measures of health. The aim of this thesis was to examine the association between nutrition-related factors (especially diet quality) and HRQOL in community-dwelling older adults aged 60 and above. This research used cross-sectional data from the National Health and Nutrition Examination Survey (NHANES) 2009-2010 and 2011-2012 cycles to select and include diet quality variables, meal-related variables, and other relevant covariates in regression models, which predicted HRQOL measures in a nationally representative sample of older adults $(n=3,692)$. The analysis of the association between diet quality and HRQOL and the adjustment for covariates was performed with logistic regression using SAS (Statistical Analysis Software), version 9.4. All analyses were weighted using appropriate NHANES weights so that findings were nationally representative. Compared to older adults in the lowest quartile for HealthyEating Index (HEI) scores, older adults in the highest quartile for HEI scores had 2.4 times greater odds of good or better HRQOL compared with fair or poor HRQOL (95\% Cl 1.55 to $3.56, p<0.0001)$. Overall, in multiple models and approaches, diet quality was a significant predictor of better HRQOL after adjusting for meal-related and other covariates, indicating an association between these two variables. Investigations of HEI as a predictor of physical and mental healthy days measures (0-15 days vs. $16-30$ days per month) did not reveal any significant relationships, although meal prep difficulty and poor appetite/overeating were significant predictors of HRQOL. More research is needed 
to determine the cause-and-effect relationship between diet and self-perceived HRQOL, and how diet can impact overall HRQOL over time. 


\section{ACKNOWLEDGMENTS}

Most importantly, I would like to thank Dr. Louise Berner, for her invaluable guidance, expertise, and endless support throughout my entire thesis research process. I would also like to thank my committee member and committee chair, Dr. Lisa Nicholson and Dr. Kari Pilolla, for their support in my nutrition classes as well as their advice while I was compiling my thesis research.

I would also like to thank my committee member, Dr. Ulric Lund, for using his statistical knowledge to help improve my SAS code and my statistical models. I would also like to thank him for being a great professor in the SAS programming class I took before starting my research, which gave me the knowledge I needed to manage the data I was analyzing.

Last but certainly not least, I would like to thank my family and friends for their continuous support and encouragement during the process of completing my Master's degree. 


\section{TABLE OF CONTENTS}

Page

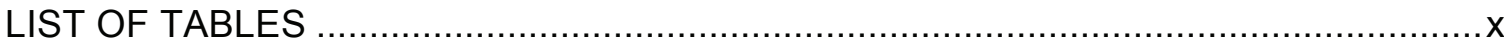

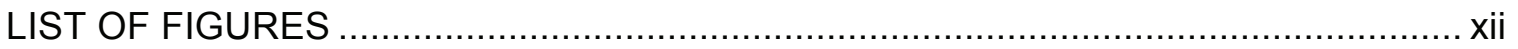

CHAPTER

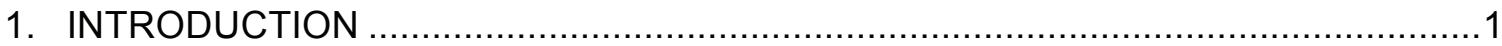

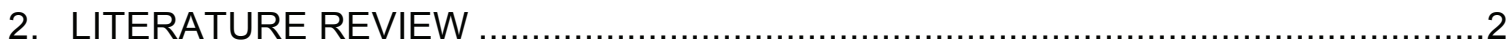

2.1 Older Adult Population in the United States.............................................

2.2 Diet Quality and Nutritional Status in

Community-Dwelling Older Adults ......................................................... 8

2.2.1 Predictors of Diet Quality and

Nutritional Status in Community-Dwelling

Older Adults ...................................................................... 10

2.2.2 Programs Designed to Improve Nutrition

in Community-Dwelling Older Adults .......................................12

2.3 Quality of Life: Definitions, Measures, and Predictors

in Older Adults .................................................................................... 17

2.3.1 Defining and Measuring Quality of Life and

Health- Related Quality of Life

2.3.2 Predictors of Quality of Life and Health-

Related Quality of Life in Older Adults .....................................25

2.4 Nutrition and Food in Relation to Quality of Life ......................................28

2.4.1 The Mealtime Experience in Community-

Dwelling Older Adults

2.4.2 Systematic Review, Observational, and Intervention Studies on Nutrition

and Quality of Life

2.4.3 Proposed Nutrition-Related Quality of

Life Tools

2.5 Summary, Research Needs, and

Thesis Research Questions... 


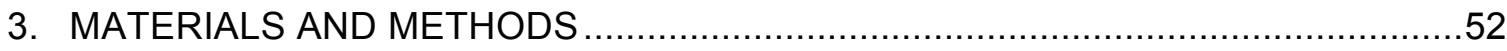

3.1 NHANES Background, Design, and Operations ...................................52

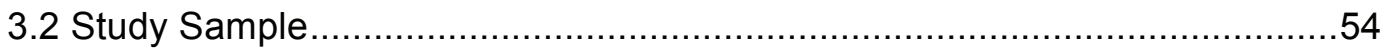

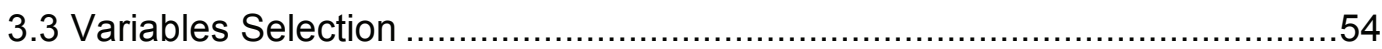

3.3.1 Health-Related Quality of Life Outcome

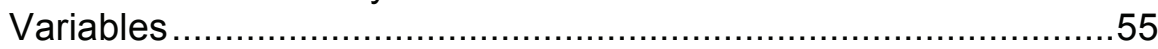

3.3.2 Diet Quality Predictor Variables ........................................57

3.3.2.1 Healthy Eating Index-2010 Overview, Background, and Uses ............................................ 58

3.3.2.2 Self-Perceived Diet Quality .......................................65

3.3.3 Other Diet-Related Predictor Variables .................................66

3.3.4 Other Predictor Variables ....................................................68

3.4 Research Questions and Statistical Analyses ......................................68

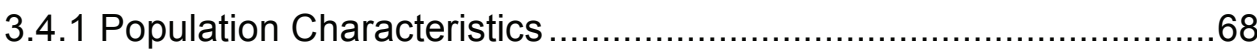

3.4.2 Diet Quality Descriptive Analyses.........................................70

3.4.3 Regression Models ........................................................ 71

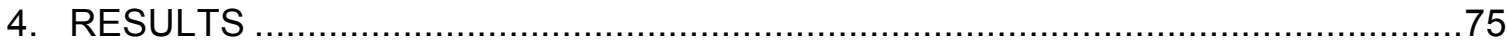

4.1 Population Descriptive Data.......................................................... 75

4.2 Diet Quality Predictor Variables: HEI and Self-Perceived

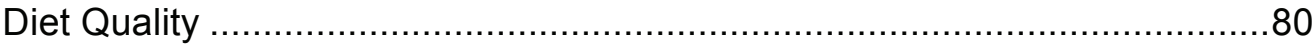

4.3 Other Diet-Related Predictor Variable Data........................................... 85

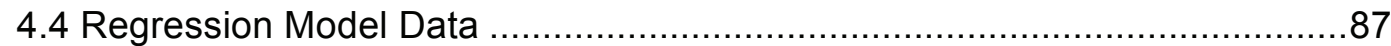

5. DISCUSSION, CONCLUSIONS, AND FUTURE RESEARCH ..........................101

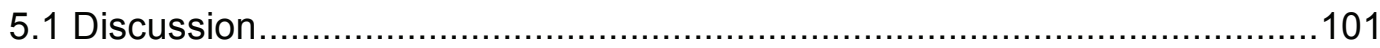

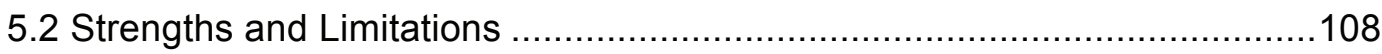

5.3 Conclusions and Suggestions for Future Research.............................111 


\section{APPENDICES}

A. Short Form-36 Survey Instrument for Measuring Quality of Life .124

B. Short-Form 36 Scales and Associated Questions

C. CDC's Healthy Days Questionnaire for Measuring Health-Related Quality of Life

D. CDC's Activity Limitations Questionnaire for Measuring Health-Related Quality of Life

E. CDC's Healthy Days Symptoms Questionnaire for Measuring Health-Related Quality of Life

F. Nutrition Quality of Life Survey Version 1.4

G. Food Patterns Components in the Food Patterns Equivalents Database.

H. Modified SAS Code for the NCI Population Ratio Method to Obtain Average HEI Total Scores for Adults Aged 60+

I. Modified SAS Code to Obtain Individual Average HEI Total Scores for Adults Aged 60+ 


\section{LIST OF TABLES}

Table

2.1 Domains and Questions of the WHOQOL-BREF Tool

2.2 Categories, Domains, and Selected Items of the Sickness

Impact Profile (SIP)

2.3 A Summary of 5 Studies Examining the Relationship Between Nutrition and Quality of Life

2.4 Statistically Significant Associations Between Total Diet Scores and Quality of Life Domain after 5 Years

2.5 Quality of Life Factors (QF) Questionnaire Domains, Number of Items, Selected Items, and Likert-Scale Response Type

2.6 Proposed Items for Measuring Satisfaction with Food-Related Life

2.7 Items in Schunemann and Others' Nutrition-Related Quality of Life Tool

3.1 Healthy Eating Index-2010 Components and Scoring Standards

3.2 Possible Responses for Demographic Predictor Variables, NHANES 2009-2012.

4.1 Demographic Information of Older Adults 60+ by Age Group in NHANES Data Cycles 2009-2010 and 2011-2012

4.2 Weighted Percentages of Health-Related Quality of Life Response Categories by Demographic Characteristics of Older Adults $60+$ in NHANES Data Cycles 2009-2010 and 2011-2012

4.3 Average Mean Healthy Eating Index-2010 Total Scores Obtained Using the $\mathrm{NCI}$ Population Ratio Method

4.4 Average Healthy Eating Index-2010 Total Scores and Standard Errors by Health-Related Quality of Life Category Using the $\mathrm{NCl}$ Population Ratio Method in Adults aged 60+ for the NHANES Survey Cycles 2009-2010 and 2011-2012.

4.5 Average Healthy Eating Index-2010 Total Scores by Self-Perceived Diet Quality Category Using the NCI Population Ratio Method from Older Adults 60+, NHANES Data 2009-2010 and 2011-2012 
4.6 Unweighted Percent of Subjects for Healthy Eating Index-2010 Scores Day 1 and Day 2 Quintiles from NHANES Dietary Data 2009-2010 and 2011-2012

4.7 Individual Average Daily Healthy Eating Index-2010 Scores, Average Over Two Days, and 2-Day Average Healthy Eating Index-2010 Total Scores of Older Adults 60+ from NHANES Data 2009-2010 and 2011-2012

4.8 Self-Perceived Diet Quality Weighted Percentages of Older Adults $60+$ by Age Groups

4.9 Average Individual Healthy Eating Index-2010 Scores Over

Two Days by Self-Perceived Diet Quality Response Category

4.10 Weighted Percentages of Other Diet-Related Predictor Variables of Older Adults 60+ by Age Group from NHANES Data Cycles 2009-2010 and 2011-2012

4.11 Adjusted Odds Ratios for the Continuous HEI Model, the Categorical HEI Model, and the Self-Perceived Diet Quality Model Predicting Good or Better HRQOL

4.12 Adjusted Odds Ratios of Healthy Eating Index-2010 Average Total Scores Over Two Days by Meals Eaten and Poverty Responses.

4.13 Adjusted Odds Ratios for the Continuous HEI Model Predicting Physically Unhealthy Days, Mentally Unhealthy Days, and Inactive Days due to Physical/Mental Health Issues 


\section{LIST OF FIGURES}

Figure

2.1 Older Adult Population (in millions), Aged 65-85+, from 1900-2010 and Projected 2020-2050. From US Census

Bureau (2012).

2.2 Percentage of adults aged 65+ enrolled in Medicare with functional limitations according to living situation, 2009.

From FIFARS (2012).

4.1 Scatterplot of the Correlation Between Healthy Eating Index-2010 Total Scores for Day 1 and Day 2, Calculated from NHANES Dietary Data, 2009-2010 and 2011-2012 


\section{Chapter 1: Introduction}

Health-related quality of life (HRQOL) is defined as the physical, mental, social, and emotional aspects that impact overall self-perceived health (Centers of Disease Control and Prevention [CDC], 2011a). HRQOL and well-being is a new topic area of Healthy People 2020, indicating its importance as a public health concern (Office of Disease Prevention and Health Promotion [ODPHP], 2014a). HRQOL is an essential component of the aging process due to its association with increased risk of functional impairments, chronic diseases, and institutionalization (Bernstein \& Munoz, 2012). Because of this ever-increasing age group in the United States, research has begun to focus on the older adult population and lifestyle factors that can affect overall health and well-being.

One of the main modifiable lifestyle factors is diet, and some research has indicated that diet and other meal-related variables may play a role in overall HRQOL. However, there are no reports that analyze diet quality specifically and its association with HRQOL measures in a large, nationally representative, older adult sample.

The purpose of this cross-sectional research was to examine the relationship between diet quality and HRQOL in a nationally representative sample of older adults aged 60 and above from the NHANES data cycles 2009-2010 and 2011-2012. Despite the inability to make cause and effect conclusions, this thesis research will be valuable in determining the predictive value of diet in relation to HRQOL, after adjusting for covariates relevant to the older adult population. Results will provide justification for examining diet quality as an important variable when analyzing HRQOL outcomes in future cross-sectional, cohort, and intervention studies. 


\section{Chapter 2: Literature Review}

This section provides background information on the older adult population, as well as aspects of diet quality, ways to measure quality of life, and the relationship between diet and quality of life. In addition, future research needs and the main research questions for this thesis are described.

\subsection{Older Adult Population in the United States}

The older adult population, defined as 65 and older, is the fastest growing age group in the United States. There were 43.1 million older adults as of 2012, and that number is expected to double to 92 million in 2060 . Not only are there more adults reaching the age of 65 , but more are also living longer. In fact, older adults who turn 65 live for 19 more years, on average. Life expectancy varies by gender; women have an average life span of 81 years, while men live for an average of 76 years. Furthermore, the population aged $85+$ is estimated to triple in number by 2040 (Administration on Aging $[A O A], 2013 a)$, as shown in Figure 2.1 below.

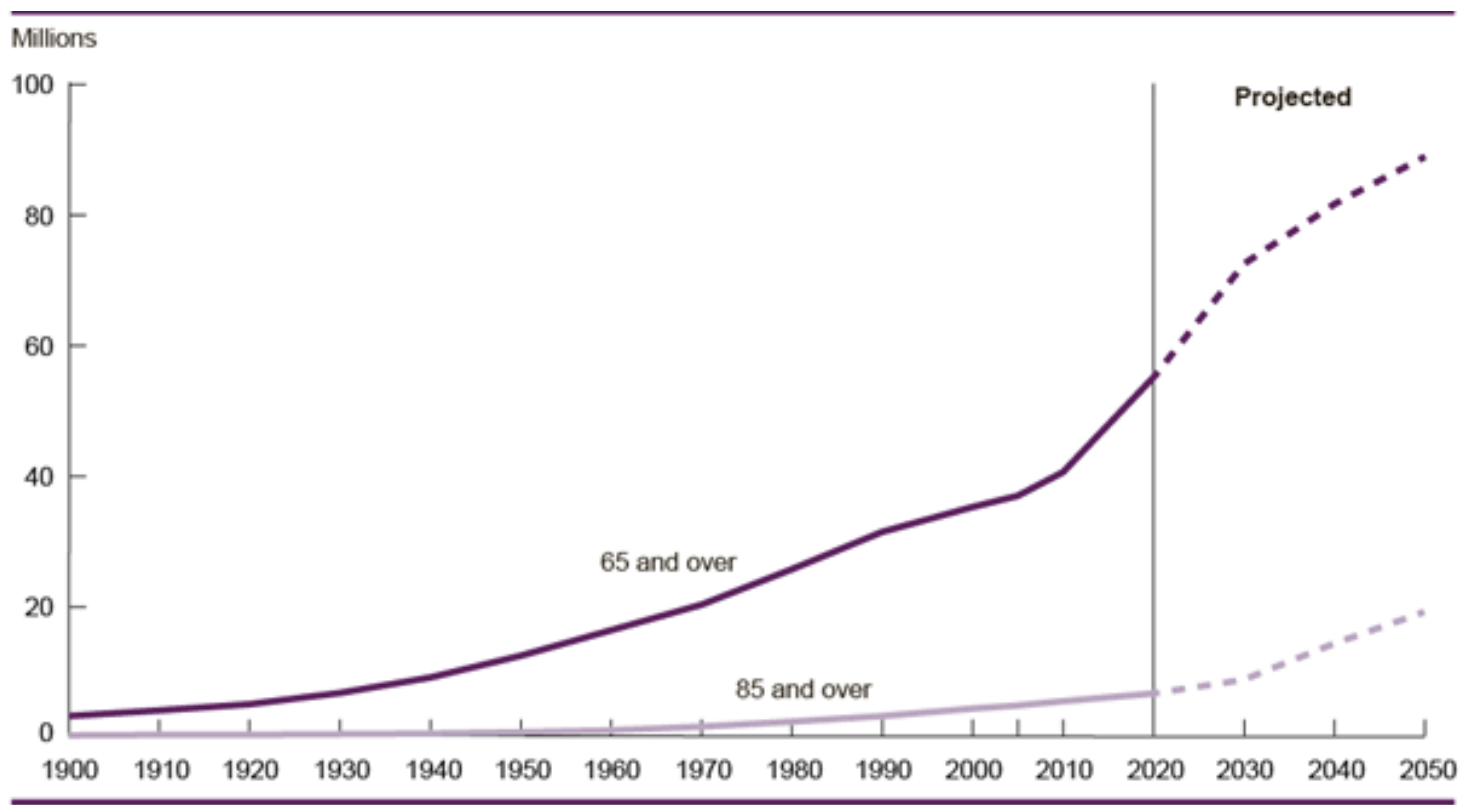

Figure 2.1 Older Adult Population (in millions), Aged 65-85+, from 1900-2010 and Projected 2020-2050. From US Census Bureau (2012). 
The older adult population consists of $57 \%$ women and $43 \%$ men, or a ratio of 129 to 100 . In the 85 and older age group, the ratio increases to 200 women for every 100 men (AOA, 2013a). There are gender differences involving marital status as well. Seventy-eight percent of men and $56 \%$ of women are married, which declines to $66 \%$ and $28 \%$ in the $75+$ population. Because of this, older women are three times more likely to be widowed compared to men (Federal Interagency Forum on Aging-Related Statistics [FIFARS], 2012). In 2010, $80 \%$ of older Americans were Caucasian, $7 \%$ were Hispanic, $9 \%$ were Black, and $3 \%$ were Asian. By 2050, the population is projected to be a more diverse group, with 58\% White, 20\% Hispanic, 12\% Black, and 9\% Asian. Educational background has improved over time, but there are differences among these racial and ethnic groups (FIFARS, 2012). As of $2010,80 \%$ of older adults had a high school diploma, and $23 \%$ had a bachelor's degree. In 1965 , on the other hand, only $24 \%$ graduated from high school and 5\% went on to attain a bachelor's degree. Older Asians received the most Bachelor's degrees, followed by non-Hispanic Whites, Blacks, and Hispanics. Higher education is positively associated with health, income, and standard of living (FIFARS, 2012).

Thirty-four percent of older adults are in the middle-income range, with the median household income equal to $\$ 31,410$ for both men and women. The largest source of revenue is Social Security, with earnings, pensions, and assets contributing to income as well (FIFARS, 2012). Money from these sources goes towards a variety of goods and services. Older adults on average spend $12 \%$ of money on food, $36 \%$ on housing, $14 \%$ on transportation, and $13 \%$ on health care. These numbers remain relatively stable across the older adult age groups, with the exception of health care expenditures; people over the age of 85 spend $3 \%$ more on health services then the younger elderly age groups. Nevertheless, spending distribution is dependent on poverty status (FIFARS, 2012). Over the past 50 years, the percent of older adults who live in 
poverty has decreased from $35 \%$ to $9 \%$. Compared to children and adults of working age, older Americans are the population group least likely to be in poverty. Despite this, aging is associated with an increased risk of functional disabilities, which can increase the risk of becoming poor. Poverty rates in older adults aged $65-74$ are equal to $8 \%$, which increases to $10 \%$ in adults aged 75 and older (FIFARS, 2012). There are also ethnic disparities involved with poverty levels. Only $7 \%$ of older Caucasians are poor, compared to $18 \%$ of African-Americans, $12 \%$ of Asians, and $21 \%$ of Hispanics (AOA, 2013a). There are gender differences as well; $11 \%$ of older women and $7 \%$ of older men live in poverty (FIFARS, 2012). The highest poverty levels based on ethnicity and gender occur among older Hispanic women (42\%) and African-American women (33\%) who live alone (AOA, 2013a).

This population lives in diverse housing arrangement. As of $2012,22 \%$ of all households have an adult aged 65+ as the householder (Vespa, Lewis, \& Kreider, 2013). Furthermore, two million elders live with at least one grandchild, and over 500,000 are the guardian in charge in a family household (AOA, 2013a). Five percent of households are multigenerational, which are families that consist of three or more generations. Compared to other family households, multigenerational households are $7 \%$ more likely to be in poverty (Vespa et al., 2013). Seventy-one percent of men and $42 \%$ of women live with a spouse or other family member, which increases to $45 \%$ among women aged 75 and older because of women's longer life expectancy. Twentynine percent of all older adults live alone (Bernstein \& Munoz, 2012). Thirty-one percent of households also have at least one unpaid family caregiver, and $70 \%$ of the caregivers are assisting adults aged 50 and older. Because of an increase in elders 75 and older, the average age of the person receiving care has also increased from 66 to 69 (National Alliance for Caregiving [NAC], 2009). 
One aspect of life that contributes to an improved lifespan and quality of life is physical activity. Exercising regularly can lower the risk of numerous chronic diseases that cause functional decline and early death, while preventing weight gain and falls. Despite this, only $11 \%$ of older adults engage in aerobic and muscle strengthening activities that meet the Federal guidelines (FIFARS, 2012). Additionally, less than 5\% participate in 30 minutes of physical activity per day, which decreases even further with age. Exercise that involves building strength, flexibility, and endurance can improve the daily life of an older adult and helps to maintain independence (Drewnoski \& Evans, 2001; Kuczmarski \& Weddle, 2005).

Functional status is an important indicator of health status and independent living. It can be determined by assessing an individual's capability to perform basic activities, which includes Activities of Daily Living (ADLs) and Instrumental Activities of Daily Living (IADLs). ADLs include bathing, dressing, toileting, getting in and out of bed, controlling urination, controlling bowel movements, and feeding oneself without assistance (Amarantos, Martinez, \& Dwyer, 2001). IADLs are more complex day-to-day activities, which consist of preparing meals, shopping, housekeeping, using transportation, using the phone, managing medications, and managing finances (Dubuc et al., 2011). Another way to assess functional status in addition to ADls and IADLs is by looking at certain aspects of physical functioning. Examples include kneeling, reaching overhead, grasping small objects, walking 2-3 blocks, and lifting heavy objects. When physical functioning was measured, $30 \%$ of women reported inability to carry out at least one of these five activities, compared to $19 \%$ of men. These percentages increase with the older age groups; $53 \%$ percent of women and $40 \%$ of men 85 and older were unable to perform at least one of these activities (FIFARS, 2012). The ability to perform ADLs and IADLs varies based on the residential setting, as shown in Figure 2.2 (FIFARS, 2012). 
According to Healthy People 2010, adults can expect to live, on average, 66 years free of activity limitations (CDC, 2010). But only $61 \%$ of community-dwelling older adults aged $65+$ can perform day-to-day activities without assistance, and nearly $40 \%$ have at least one ADL or IADL limitation (FIFARS, 2012). The leading cause of disability among elders who have difficulties with ADLs and IADLs is arthritis, which affects $59 \%$ of people 70 and older. Of the older adults with arthritis, $50 \%$ require assistance with ADLs, and 71\% need help with IADLs (Kuczmarski \& Weddle, 2005).

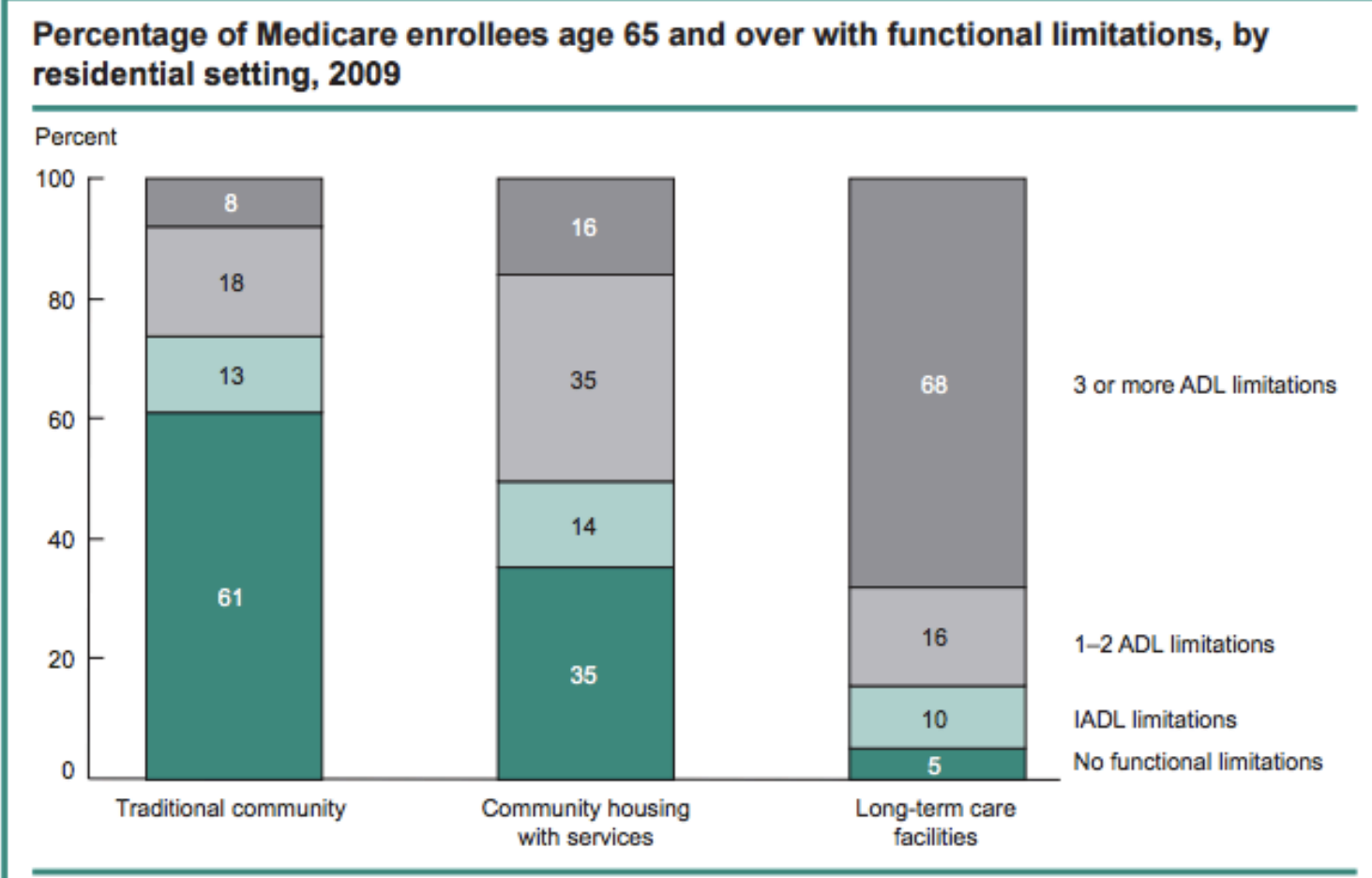

Figure 2.2 Percentage of adults aged 65+ enrolled in Medicare with functional limitations according to living situation, 2009. From FIFARS (2012).

As these functional limitations increase, the number of institutionalized older adults increases as well. In total, $3 \%$ of elders live in a long term care facility, but there are differences among age groups. One percent of adults aged $65-74,3 \%$ of adults aged $75-$ 84 , and $10 \%$ of adults $85+$ live in a care home because they require assistance (AOA, 2013a). 
Despite the $80 \%$ of older adults who self-report very good to excellent health, many have at least one chronic condition (Food and Drug Administration [FDA], 2014). The most common chronic health problems, in order of prevalence, are: hypertension, arthritis, heart disease, cancer, diabetes, asthma, emphysema, and stroke. A few of these chronic health conditions are also part of the leading causes of death among the elderly, which include: heart disease, cancer, chronic lower respiratory disease, stroke, Alzheimer's, diabetes, influenza, and pneumonia (FIFARS, 2012). Over the past 30 years, however, death rates from heart attack and stroke have decreased by $50 \%$, which is mainly because of changes in health behaviors over time and increased access to health care (Kuczmarski \& Weddle, 2005). Diabetes, on the other hand, is not decreasing; more than $25 \%$ of older adults have type 2 diabetes. The CDC estimates the prevalence of type 2 diabetes to double in the next 20 years, partly because of the growing elderly population. Diabetic complications put older adults at a higher risk for functional impairment and institutionalization, which is why screening and other preventive measures need to take place early on (Kirkman et al., 2012). Another disease that contributes to low functional status and dependent living is Alzheimer's. An estimated 5 million older adults have this illness as of 2014 , with the majority ( $82 \%)$ in the 75 and older age group (Alzheimer's Association [AA], 2014).

Chronic illnesses are long-term and rarely curable, and create a heavy cost burden on the health care system. In 2008, $\$ 368$ billion dollars were spent on caring for the elderly, with heart conditions as the most common diseases (Soni \& Roemer, 2011). Health care costs increase with age as well. Older adults between the ages of 65 and 74 on average cost $\$ 7,000$ annually to treat, while adults over the age of 85 cost $\$ 19,000$, on average. Even though $93 \%$ of older adults have Medicare for health insurance coverage, 94\% had to pay out-of-pocket for health care services (FIFARS, 2012). 
The older adult population is a rapidly growing age group, and is projected to represent $20 \%$ of the U.S. population by 2030 . A major contributor of the aging population is the "Baby Boomer" generation, members of which began turning 65 in 2011. The "oldest old" age group of 85 and older is expected to grow to 19 million by 2050 , but death rates at older ages are expected to decline more rapidly because of improved health care and nutrition services (FIFARS, 2012). However, older adults with functional impairments in at least one IADL or ADL are more likely to live in poverty compared to older adults who are able to take care of themselves. Functional status can have a major impact on quality of life, health, chronic illness, and nutritional status. Because functional limitations tend to increase with age, it is important examine all the factors involved in order to maintain independence among the older adult population (FIFARS, 2012).

\subsection{Diet Quality and Nutritional Status in Community-Dwelling Older Adults}

Nutritional status is the condition of a person that is influenced by nutrient intake and utilization, which greatly contributes to an older adult's health and functionality (Amarantos et al., 2001). Consuming an excessive or insufficient amount of calories puts people at risk for chronic illnesses (Deirelein et al., 2014). In fact, two-thirds of the older adult population does not consume the recommended amount of calories, vitamins, and minerals as part of a daily diet (Bernstein \& Munoz, 2012). Inadequate energy and protein consumption leads to a reduction in muscle mass and strength, which can increase the occurrence of falls and impair functional status. In addition, the ability to fight infection can become compromised because inadequate protein intake can weaken immune function (Allard, 2001). Overall energy intake tends to decrease with age, which can also lead to a decrease in nutrients. In particular, older adults are at risk for low levels of iron, calcium, vitamin D, vitamin E, vitamin B12, potassium and zinc. Vitamin B12 levels in older adults are inadequate mainly because of malabsorption; the other 
vitamins and minerals previously listed are insufficient due to intake (Bernstein \& Munoz, 2012).

Diet quality is a strong indicator of nutritional status and nutrient intake in older adults. Dietary quality can be assessed using the Healthy Eating Index 2010 (HEl-2010), which was developed by the USDA Center for Nutrition Policy and Promotion (United States Department of Agriculture Center for Nutrition Policy and Promotion, [USDACNPP], 2014). This index measures compliance with 12 components of the Dietary Guidelines for Americans 2010. A higher score indicates a high quality diet, which can reach a maximum of 100 points (FIFARS, 2012). The food categories in the adequacy component of the HEI-2010 are: total fruits, whole fruit, total vegetables, greens and beans, whole grains, dairy, total protein foods, seafood and plant proteins, and fatty acids. Sodium, refined grains, and empty calories are part of the moderation component. Standards for maximum and minimum scores are based on the 2010 Dietary Guidelines. For foods in the adequacy component, intakes at or above the standard level receive the maximum amount of points, and an intake of zero receives a score of zero. For items in the moderation component, intakes at or below the standard level receive the maximum amount of points, and the minimum score is based on percent energy intake (Guenther et al., 2013). HEI scoring is described more fully in the Materials and Methods Chapter of this thesis.

Prior versions of the HEI were developed for earlier sets of Dietary Guidelines. The number of adequacy and moderation components remained the same in both indexes, but a few of the food categories changed. The food groups in the HEI-2005 that are not seen in the 2010 version include dark green vegetables, orange vegetables, and legumes, total grains, milk, meats and beans, oils, saturated fat, and calories from solid fats, alcoholic beverages, and added sugars (Guenther et al., 2013). A study conducted by Deierlein and colleagues (2014) analyzed diet quality scores of 1,300 independent 
older adults in the New York City area. Forty percent of the participants were between the ages of 60 and $70,38 \%$ were $71-80$ years old, and $22 \%$ were $81-99$ years old.

Results indicated that the total HEI mean diet quality scores were 72 for women and 69 for men. Diet quality scores were not separated by age; therefore, it is difficult to distinguish possible discrepancies between the age groups. Nevertheless, scores in this range suggest a need for improvement (Deirelein et al., 2014). On a larger scale, the USDA discovered that the fruit, total grains, meats, and beans categories were of the highest quality in an older adult's diet. Saturated fats, alcohol, sodium, and added sugar intake were high, and therefore lowered the overall diet quality score, according to the HEI-2005 (FIFARS, 2012). Older adults who consumed higher amounts of added fats, sugar, and sodium tended to under consume fiber, calcium, magnesium, potassium, zinc, folate, and vitamins A, B6, C, D, and E. This puts older adults at a greater risk for poor nutritional status and obesity as a result (Kamp, 2010). Because of the key role of food and nutrient intakes in maintaining health, it is important to investigate key predictors of diet quality and good nutritional status.

\subsubsection{Predictors of Diet Quality and Nutritional Status in Community-Dwelling Older Adults}

One predictor of good nutritional status in older adults is food security, which is defined as having access to adequate and nutritious foods at all times (Lee \& Frongillo, 2001). Currently, $6 \%$ of households with older adults have experienced some form of food insecurity that has prevented adequate intake of foods. Food insecurity is more prevalent in Hispanic (15\%) and African Americans (19\%) compared to Caucasian elders (4\%), which can be attributed to lower incomes among the minority groups (Kuczmarski \& Weddle, 2005). In fact, $26 \%$ of all older adults are in the low-income group (Kamp, 2010), 9\% are below the poverty level, and an additional 5\% are considered "near-poor" (Bernstein \& Munoz, 2012). Those that are poor and near-poor 
are three times more likely to be food insecure compared with older adults who live above the poverty line (Ziliak \& Gundersen, 2011). People in the low-income category tend to eat lower amounts of fruits, vegetables, dairy, lean, high-quality protein and overall calories than adults with higher incomes, indicating an association between diet and income (Wunderlich, Brusca, Johnson-Austin, \& O’Malley, 2012).

Poor functional status and poor nutritional status are directly related to each other in a circular fashion. If older adults are not consuming adequate amounts of highquality protein, muscle mass will decline, which leads to frailty and impaired mobility (Allard, 2001). A poor quality diet low in antioxidants, for example, can lead to an inflammatory response, which can exacerbate a chronic condition and affect functional ability (Bernstein \& Munoz, 2012). Lower quality diets are associated with lower extremity immobility (Gopinath, Russell, Flood, Burlutsky \& Mitchell, 2014). At the same time, disabled seniors who are incapable of carrying out IADLs such as food shopping, driving or taking public transportation are at a greater risk of insufficient food intake (FIFARS, 2012). Even if older adults are able to buy food independently they may have difficulties preparing and cooking meals, and require assistance from a family member (Kamp, 2010).

Living arrangements can also have an impact on nutritional status. Older adults who live with a spouse consume a higher quality diet compared to elders who live alone. Specifically, men who live alone tend to have a tougher time preparing meals, while women are less likely to cook and prepare food for themselves (Deirlein et. al, 2014). Widowhood, which occurs in $36 \%$ of older women, can also have a negative effect on food intake (AOA, 2013a). Older adults who live alone are at an increased risk of depression, which can lower appetite and food consumption as a result. Furthermore, management of an elder's diet may be more difficult if there are no other family members present (Payette \& Shatenstein, 2005). Caregivers who live in the household 
can help with food shopping, meal preparation, and feeding if an older adult is unable to carry out activities independently. However, caregivers may lack the nutritional knowledge to choose foods that provide adequate amounts of nutrients, which is why nutrition education and counseling may be beneficial in improving diet quality (Bernstein \& Munoz, 2012).

Grocery shopping provides a source of social interaction for older adults, but there are barriers to a satisfactory food shopping experience (Elsner, 2002). The size of a supermarket can be off-putting to elders because it is challenging to walk around and find certain foods. Some older adults even feel that they are a burden to other shoppers and staff at grocery stores, and do not feel comfortable asking for assistance when needed. Food items that are placed too high or low on the shelves may be out of reach, making it difficult to choose and purchase items. Reading food labels can also pose a problem. Part of the natural aging process involves poor vision, which can make it impossible to read small print on various food items (Meneely, Strugnell, \& Burns, 2009). Inability to shop for food can greatly affect an older adult's health. If seniors cannot buy food they may resort to convenience foods that are higher in fat, calories, and sodium (Holmes, 2006). Since two thirds of older adults do not follow the recommended dietary guidelines and are therefore consuming low quality diets, government programs may be helpful in improving nutritional status (Bernstein \& Munoz, 2012).

\subsubsection{Programs Designed to Improve Nutrition in Community-Dwelling Older}

\section{Adults}

The Elderly Nutrition Program (ENP), created under Title III-C of the Older Americans Act, is the largest program that focuses on providing nutrition services for older adults (Gollub \& Weddle, 2004). Services include congregate meals, homedelivered meals, nutrition education, and counseling. Congregate nutrition services, established in 1972, provide meals in congregate facilities in community locations, as 
well as a source of social interaction to participants. In 2010, congregate nutrition services provided 96.4 million meals to 1.7 million older adults, while home-delivered nutrition services provided 145.4 million meals to 868,076 participants (AOA, 2013b). Overall, 236 million meals are served to 2.6 million elders per year, which is less than $5 \%$ of the older adult population (Kamp, 2010). Health services also offered to older adults in need consist of medical screenings, homemaker assistance, and social activities. Anyone over the age of 60 is eligible for congregate meals, but the ENP's emphasis is on adults who are frail, homebound, and therefore unable to buy and prepare food independently (Millen et al., 2002).

Home-delivered meals assist homebound older adults who may not be receiving proper nutrition on a daily basis when functional limitations interfere with meal preparation and cooking (Anyanwu et al., 2011). In fact, $41 \%$ of homebound older adults who participate in the ENP are unable to prepare meals, and $85 \%$ have difficulties with at least one IADL (Wellman, Rosenzweig, \& Lloyd, 2002). However, nearly half of the meal delivery programs across the United States have waiting lists, preventing many elders from joining (Anyanwu et al., 2011). The home-delivery meal program benefits older adults in numerous ways. The foods provide nutrients that elders may not be consuming independently, while the people delivering the foods provide a source of social interaction. The ENP also provides nutrition education and counseling to promote nutrition knowledge among older adults (Bernstein \& Munoz, 2012). Part of nutrition education involves teaching program participants proper diet and exercise behaviors that will either prevent or keep chronic illnesses from progressing (Thomas, Ghiselli, \& Almanza, 2011).

Older adults who are nutritionally at risk for certain health problems can benefit greatly from a food assistance program (Kamp, 2010). The meals provided by the ENP make up $30-50 \%$ of the participant's daily nutrient intakes, which is significant for older 
adults who are unable to prepare nutrient dense foods independently (Millen et al., 2002). Frongillo and colleagues (2010) discovered that older adults who are part of a home-delivered meal program had a higher intake of fruits and vegetables compared to nonparticipants. Elders tended to be deficient in vitamins found in fruits and vegetables, which suggests that meal programs can improve nutritional status (Bernstein \& Munoz, 2012).

In addition to congregate and home-delivered meals, the Supplemental Nutrition Assistance Program (SNAP) is available to older adults. Previously known as the Food Stamp Program, SNAP helps low-income individuals and families purchase foods in order to maintain a healthy diet. As of 2012 , SNAP served 47 million people per month, and $9 \%$ of those people were older adults (United States Department of Agriculture Food and Nutrition Services [USDAFNS], 2014a). Elders receive fewer benefits compared to adults and children because they do not qualify according to the asset tests, thereby lowering the application rate. In addition, older adults who live in rural areas also may find it difficult to travel to SNAP office locations in order to sign up for its nutrition services, which can prevent eligible elders from applying (USDAFNS, 2014). Current research has shown that the relationship between SNAP and food security is affected by the household situation. For example, SNAP has a positive effect on food security in households with or without children, a disabled family member, and without an older adult. Households that only contain older adults without earnings participate in SNAP significantly longer than households without elders (United States Department of Agriculture [USDA], 2013). Research is needed to determine reasons why food security in elders is unaffected by SNAP participation, while it has proven to be effective in children and adults.

The Commodity Supplemental Food Program (CSFP) provides USDA foods specifically to low-income older adults in order to improve nutritional status. In 2013 , 
more than 579,000 older adults participated per month, which is only $1.3 \%$ of the U.S. population (USDAFNS, 2014b). The CSFP provides both home-delivered and congregate food packages, which consist of canned fruits, vegetables, meat or fish, juice, instant dry milk, pasta or rice, dry beans or peanut butter, and cereal. Older adults are eligible if they are at or below $130 \%$ of the Federal Poverty Income Guideline (National Commodity Supplemental Food Programs Association [NCSFPA], 2013). Research regarding the CSFP and its effects on nutrition and health in older adults has not been conducted on a national scale. The most recent research done was at a state level in 1993, in which CSFP participants in New Orleans, Louisiana were evaluated based on anthropometrics. This study, conducted by Koughan and Atkinson (1993), involved 104 older adults, aged 60 and above. Heights and weights were measured, along with a screening checklist to assess nutritional risk. Results indicated that $80 \%$ of participants were at moderate to high nutritional risk based on the screening tool. The median body mass index (BMI) was 30 , which is classified as obese. Of the elders with this BMI value, $50 \%$ were at a high nutritional risk, which can be damaging to health and nutritional status (CSFP, 2014). Current research on nutritional risk and CSFP should occur in order to determine food program effectiveness over time.

Older adults below $185 \%$ of the Federal Poverty Income Guidelines are eligible to participate in the Senior Farmer's Market Nutrition Program (SFMNP), which is a relatively new food and nutrition program created by the USDA in 2004 . The SFMNP provides coupons for low-income older adults that can be exchanged for fresh fruits, vegetables, honey, and herbs at Certified Farmer's Markets. Over 800,000 (1.9\%) of seniors participated in the program in the fiscal year 2013, with 20,000 farmer's markets available to them (USDAFNS, 2014c). Once a year, seniors receive a coupon booklet ranging in value from $\$ 20$ to $\$ 50$ to use at farmer's markets from May to November, due to seasonal food availability (California Department of Food and Agriculture [CDFA], 
2014). There is limited research on the SFMNP and its impact on older adults. A study conducted by Kunkel, Luccia, and Moore (2003) involved evaluating a SFMNP in South Carolina. The researchers mailed out a survey to a random sample of 1,500 older adults participating in the SFMNP, and 658 surveys were completed and returned. Overall, $64 \%$ of participants reported that having the coupons to spend at farmers' markets has changed the way they eat. Seniors also indicated the primary reason they did not use coupons was because there were no farmer's markets in their area, creating a barrier to participating in the program. Eighty-six percent stated they eat more fresh fruits and vegetables because of the SFMNP, and $56 \%$ said they learned a new way to prepare or cook these foods. Based on this research, it appears that the SFMNP is associated with improved eating habits and therefore nutritional status in older adults (Kunkel, Luccia, \& Moore, 2003). Due to the relatively small scale of this study, however, further and more up-to-date research is needed to fully assess SFMNP effectiveness.

Despite the benefits that food and meal programs can offer to seniors, accessibility and participation across the United States is limited. Some older adults are unaware of the programs that are available, while others believe the application process is too complicated and not worth the effort. Elders also feel that there is a social stigma associated with receiving government aid, and they believe support is not necessary as a result (Bernstein \& Munoz, 2012). In addition, food programs may not be available in certain locations due to inadequate funding, which contribute to low participation rates. Increasing awareness of the Elderly Nutrition Program and adjusting the sign up process to make it less complex for older adults are just a few simple ways to improve the program and overall participation rates (Choi, Lee, \& Goldstein, 2011). Also, waitlists occur in $35 \%$ of home-delivered meal programs, making it difficult for older adults to participate in the first place. Funding for the ENP has not increased with the increasing demand for meals, which is the main cause of waitlists. Furthermore, there is little 
research on the demographics of program participants, especially those who are put on the waitlist to receive meals (Lee et al., 2011).

Nutrition and food intakes in older adults have been documented, which typically involve objective measures to assess results. Nutritional status can be measured objectively using blood and urine tests, while diet quality can be assessed using the Healthy Eating Index (Bernstein \& Munoz, 2012; USDACNPP, 2014). Predictors of nutritional status and diet quality, such as food security, functional status, and living arrangements, are usually measured subjectively using surveys in an interview setting (FIFARS, 2012). The few studies documenting meal program effectiveness utilized both objective and subjective measurements to analyze data. Frongillo and colleagues (2010), for example, measured the height and weight of each participant, along with a 24-hour dietary recall and food-insecurity questionnaire to gain subjective insight from the individuals (Frongillo \& Wolfe, 2010). Koughan and Atkinson, researchers analyzing older adults in the CSFP, used a nutritional risk screening checklist in addition to height and weight measurements (CSFP, 2004). However, all of these factors related to nutrition do not take one important measurement into account, and that is quality of life. A person's quality of life, generally defined as overall life satisfaction, can be greatly impacted by diet quality and nutritional status, which is why it is an important variable to explore.

\subsection{Quality of Life: Definitions, Measures, and Predictors in Older Adults}

Quality of life is a multidimensional concept that measures a person's perception of overall health and wellbeing (Amarantos et al., 2001). Not only is quality of life measured on an individual level, but it can also be assessed from a societal and community perspective (Felce \& Perry, 1995). Looking at quality of life in all people of the United States allows the implementation of policies tailored to improving the wellbeing of the general public (CDC, 2011b). However, the subjective, self-reporting 
nature of quality of life makes it a difficult concept to describe and measure in a research setting. The purpose of this section is to discuss the various definitions, ways to measure, and predictors that can have an impact on quality of life.

\subsubsection{Defining and Measuring Quality of Life and Health-Related Quality of Life}

Quality of life is generally described as overall life satisfaction, as well as the ability to enjoy day-to-day activities (Drewnowski \& Evans, 2001). Since quality of life is prevalent in all aspects of daily living, Healthy People has made it an overarching goal over time. When Healthy People 2000 first released, the main goal was to increase the span of healthy life, regardless of the quality of life in those years. As quality of life became more prevalent in research studies, both Healthy People 2010 and Healthy People 2020 made its primary goal of increasing quality and years of healthy life, instead of only focusing on life span and mortality measures (CDC, 2010).

Another, more specific aspect of quality of life is health-related quality of life, which focuses solely on how health status can affect life satisfaction (ODPHP, 2014a). This concept still contains domains related to physical and mental well-being, but also looks at how a disease or the aging process can alter the way someone perceives their quality of life (Amarantos et al., 2001). The definition of health is more than just the absence of illness; it also includes the well-being of the body and mind (World Health Organization [WHO], 2003). Health-related quality of life can be used to look at mental and physical factors associated with the broader quality of life, how it differs among individuals, and how it can be linked to the health care system. A screening tool can also be used to measure adults with chronic illnesses (Amarantos et al., 2001). To accommodate this growing interest in quality of life, health-related quality of life, and its affects on longevity, the number of measurement tools specific to quality of life domains needs to increase as well. 
There are various ways to measure quality of life; typically, it is translated into numerical scores that are derived from assessment tools. The WHO, for example, has a 100-item quality of life tool that consists of 4 broad categories: physical health, psychological health, social relationships, and environmental issues (WHO, 2014). Physical health includes ADLs, mobility, and pain, while psychological health involves feelings and self-esteem. Social relationships consist of the frequency and number of social contacts present, and environment relates to finances, health care, and living situation (Drewnowski \& Evans, 2001). Examples of questions in each of these domains are shown in Table 2.1.

Table 2.1 Domains and Questions of the WHOQOL-BREF Tool

Domains

Overall Quality of Life and General Health

Physical Health

Psychological

Social Relationships

\section{Questions}

How would you rate your quality of life?

How satisfied are you with your health?

To what extent do you feel that physical pain prevents you from doing what you need to do?

How satisfied are you with your ability to perform your daily living activities?

How much do you enjoy your life? How often do you have negative feelings such as blue mood, despair, anxiety, depression?

How satisfied are you with your personal relationships? How satisfied are you with the support you get from your friends?

Adapted from University of Washington (2011).

A common quality of life assessment tool, which has been seen in over 400,000 publications, is the Short Form-36. This form consists of 36 items, including physical and mental aspects, which are described in Appendix A (Carson, Hidalgo, Ard, \& Affuso, 2014). These measures are broken down into 8 scales, which involve physical functioning (PF), role-physical (RP), bodily pain (BP), general health $(\mathrm{GH})$, vitality $(\mathrm{VT})$, social functioning (SF), role-emotional (RE), and mental health $(\mathrm{MH})$, as shown in Appendix B. Each of these scales has specific items associated with them, which add up 
to 36 questions total (Ware, 2000). These items are standardized on one scale, where a higher score indicates higher physical status and mental status in relation to quality of life (Gallegos et al., 2009). The SF-12, a shortened version of the SF-36, is also used to assess quality of life. The SF-12 has the same 8 domains as the SF-36, but it uses composite scores for physical and mental health rather than individual scores per item (Raad, 2014). However, the primary authors do not recommend the SF-12 due to the fact that only summary scores are used, making it difficult to look at the range of scores in each domain (Haywood et al., 2004). Even though these short forms are considered generic tools, they can be distributed to specific age groups. Since the SF-36 consists of items such as daily physical functioning and bodily pain, older adults tend to be the population targeted for analysis (Ware, 2000).

Another generic assessment tool used in research is the Quality of Life Index (QLI), which was developed in the United States in 1984 (Haywood, Garrat, \& Fitzpatrick, 2004). The QLI measures quality of life in relation to satisfaction and importance of each of the 4 domains, which include family, health/functioning, psychological/spiritual, and social/economic. These domains produce four individual scores in addition to an overall quality of life score. Scores are placed on a Likert scale from 1-6, with 1 being 'very dissatisfied' or 'very unimportant,' and 6 being 'very satisfied' or 'very important.' Total scores range from 0-30, with higher scores indicating a higher quality of life. The QLI was originally tested on dialysis patients, but it has only been evaluated once in an older adult population (Haywood, Garrat, \& Fitzpatrick, 2004).

A quality of life tool specifically geared towards older adults aged $65+$ is the Comprehensive Assessment and Referral Evaluation (CARE). Health-related quality of life is assessed using 4 domains, which include psychiatric, medical/physical/nutritional, social needs, and service needs. This tool is comprised of 1500 items, thus requiring a trained interviewer to administer the questionnaire (Haywood et al., 2004a). It also lacks 
evidence regarding test-retest reliability, thereby limiting its usage in future studies (Haywood, Garrat, \& Fitzpatrick, 2004). A shorter version of this tool called CORECARE, on the other hand, has been tested for reliability and validity in several types of studies. It is characterized by 6 domains, which are depression, dementia, disability (activity limitation), subjective memory, sleep, and somatic symptoms. Scores are added together based on the 4 domains previously described in the longer CARE version. CORE-CARE also requires a trained interviewer to carry out the survey, but it is less time consuming compared to the original CARE tool (Haywood et al., 2004).

There are a variety of different tools that can be used to assess quality of life based on similar domains and subgroups. There are generic measurements that can be utilized for any age group, as well as age-specific tools that target a particular population (Haywood et al., 2004). Each tool was evaluated in one form or another, but there are some limitations involved as well. Some were only evaluated one time with one population, while others have inadequate evidence regarding reliability and validity (Haywood et al., 2004). This is also true for assessment tools that are related to certain diseases and health status (Carson et al., 2014).

Health-related quality of life (HRQOL) can be measured in various ways. The most common way to assess HRQOL is by calculating the number of healthy days per month, which is subjective and self-reported (Drewnowski \& Evans, 2001). The CDC has their HRQOL-14 "Healthy Days Measures" in their research, which involves 14 questions divided into 3 categories. The first tool is the Healthy Days Core Module, which includes 4 questions asking about mental and physical health in the past 30 days (see Appendix C). The second set of questions is under the Activity Limitations Module, which consists of 5 questions asking about physical, mental, or emotional limitations in the past 30 days (see Appendix D). The last category is the Healthy Days Symptoms Module, which involves questions regarding pain, emotions associated with depression, 
and insomnia, as shown in Appendix E. These modules have been utilized in the National Health and Nutrition Examination Survey (NHANES), the Behavioral Risk Factor Surveillance System (BRFSS), and the Medicare Health Outcome Survey (HOS) (CDC, 2012).

Another tool used to measure health-related quality of life is the Sickness Impact Profile (SIP), which was developed in 1976, and later revised in 1981 (American Thoracic Society, [ATS], 2007). This generic tool consists of questions that assess quality of life based on disability or illness; the categories, domains, and selected items from each are listed in Table 2.2.

Table 2.2 Categories, Domains, and Selected Items of the Sickness Impact Profile (SIP)

\begin{tabular}{|c|c|c|}
\hline Categories & Domains & Example item within each domain \\
\hline \multirow[t]{3}{*}{ Physical } & Ambulation & I walk shorter distances or stop to rest often \\
\hline & Mobility & I stay within one room \\
\hline & $\begin{array}{l}\text { Body care and } \\
\text { movement }\end{array}$ & $\begin{array}{l}\text { I do not bathe myself at all, but am bathed by } \\
\text { someone else }\end{array}$ \\
\hline \multirow[t]{8}{*}{ Psychosocial } & Social interaction & $\begin{array}{l}\text { I isolate myself as much as I can from the rest of } \\
\text { the family }\end{array}$ \\
\hline & Communication & I am having trouble writing or typing \\
\hline & Alertness behavior & $\begin{array}{l}\text { I have difficulty reasoning and solving problems, for } \\
\text { example, making plans, making decisions, learning } \\
\text { new things }\end{array}$ \\
\hline & $\begin{array}{l}\text { Emotional } \\
\text { Behavior }\end{array}$ & I laugh or cry suddenly \\
\hline & Sleep and rest & I sit during much of the day \\
\hline & Eating & $\begin{array}{l}\text { I am eating no food at all, nutrition is taken through } \\
\text { tubes or intravenous fluids }\end{array}$ \\
\hline & $\begin{array}{l}\text { Home } \\
\text { management }\end{array}$ & I am not doing heavy work around the house \\
\hline & $\begin{array}{l}\text { Recreation and } \\
\text { pastimes }\end{array}$ & I am going out for entertainment less \\
\hline
\end{tabular}

Adapted from ATS (2007); Bergner, Bobbitt, Carter, \& Gibson (1981). 
Each item receives a score of 0 or 1 ("no" or "yes") that is then added up for an overall summary score, with 0 indicating poor health and 100 indicating good health (Rehabilitation Measures Database, [RMB], 2010). Participants are asked to respond to each statement based on the day the interview is carried out (Haywood et al., 2004). No special training is required to administer the interview, and it takes an average of 35 minutes to complete (RMB, 2010). This tool not only calculates scores for individuals, but can also be utilized to measure outcomes of health care and aid in policy making as well (Berger, Bobbitt, Carter, \& Gibson, 1981). Compared to other generic health-related quality of life tools, the SIP has the highest variety with regards to health domains, and has questions that are easy to comprehend and respond to (Haywood et al., 2004). These qualities are particularly important when interviewing older adults as well.

A health-related quality of life tool specified for older adults is the Older Americans Resources and Services (OARS) Multidimensional Functional Assessment Questionnaire (OMFAQ). This instrument was developed primarily to determine the impact of medical service usage on functional status in older adults. Before individuals take the OMFAQ, they are asked to complete the Short Portable Mental Status Questionnaire (SPMSQ) to determine if a proxy is necessary during the interview. There are two parts to the OMFAQ. Part A measures functional status based on five domains, which are activities of daily living/instrumental activities of daily living, economic resources, mental health, physical health, and social resources. The next part is the interview section, in which the interviewers rate each domain on a Likert scale from 1 ("functional") to 6 ("functionally impaired"). Part B consists of the Services Assessment Questionnaire (SAQ), which is used to determine health and social service needs for older adults (Haywood et al., 2004). Another tool, based off of the OMFAQ, is the Functional Assessment Inventory (FAI). It allows assessment and screening of functional status in older adults with the same 5 domains as the OMFAQ, but the SAQ is removed 
from the interview. Self-esteem and life satisfaction are added, thereby enhancing the number of items related to health status (Haywood, Garrat, \& Fitzpatrick, 2004). In regards to validity, the ADL domain was the strongest predictor of functional impairment, suggesting an association between daily activities and functional status (Haywood et al., 2004). These are only a few of the existing measures of health-related quality of life, and there are more being reviewed and evaluated both in clinical and public health settings. In fact, Healthy People 2020 is currently evaluating various measures that will help determine health-related quality of life and well-being in the U.S. population (ODPHP, 2014a).

One study conducted by Groessl and colleagues (2007) examined health-related quality of life in older adults who were at risk for disability using a different assessment tool. Mobility was assessed initially, which involved the 424 participants walking 400 meters without assistance. Physical functioning was measured using the Short Physical Performance Battery tool, which included balance, chair stands, and a 4-meter walk. These scores ranged from 0 to 12 , with 12 as the highest performance level. To determine health-related quality of life, researchers used the Quality of Well-Being Scale-Self-Administered (QWB-SA). This tool is set on a scale from $0-1$, which ranges from "death" to "optimum functioning." Results indicated that these older adults had lower QWB-SA scores compared to older adults who are not at-risk for disability, demonstrating a relationship between physical functioning and quality of life (Groessl et al., 2007).

Since health-related quality of life has a dimension focused on emotions and mental status, it is important to look at depression as a function of health and well-being in community-dwelling older adults (Gallegos et al., 2009). Health is not the only aspect that can affect quality of life; domains such as culture, values, the living situation, and career choice also have an affect. These along with many other domains make quality of 
life a difficult thing to measure in a research setting, but there is broad consensus that it needs to be measured, along with predictors as well (CDC, 2012).

\subsubsection{Predictors of Quality of Life and Health-Related Quality of Life in Older}

\section{Adults}

The environment older adults reside in can have an impact on quality of life. Baernholdt and colleagues (2012) analyzed data from the NHANES from 2005-2006 to determine health related quality of life, social functioning, and emotional well-being among 911 older adults in rural, rural adjacent, and urban areas. Health related quality of life scores were determined by compiling the total number of self-perceived unhealthy days in the past 30 days from the interview. The number of days were divided into 6 levels for scoring purposes, with $0=$ no healthy days, $1=1-5$ unhealthy days, $2=6-10$ unhealthy days, $3=11-15$ unhealthy days, $4=16-29$ unhealthy days, and $5=30$ unhealthy days. Overall, older adults aged 65+ living in rural areas had higher scores (1.31), than adjacent (1.25) areas, and urban areas (1.18), indicating slightly more unhealthy days in rural older adults. However, the small difference between these geographic locations was not statistically significant. Social functioning was determined by examining the number of close friends and the number of times older adults attended religious services. The social aspect of older adult's lives was low, with a score of 4.75 in rural areas compared to adjacent $(5.65, \mathrm{p}<0.001)$ and urban areas $(5.40, \mathrm{p}=0.002)$. This indicated to the authors that living in the countryside resulted in less social interactions and therefore close friends that could provide social support. Lack of public transportation services in these areas may also have contributed to social isolation (Baernholdt et al., 2012).

The absence of social contacts can also have an impact on health status independent of geographic isolation. Hawton and others (2011) performed a crosssectional study utilized to assess the degree of social isolation of 393 older adults living 
in a rural area of the United Kingdom, using data from the Devon Ageing and Quality of Life (DAQoL) study. The mean age of the participants was 71 years. Out of all the older adults in this study, $75 \%$ were homeowners, $47 \%$ lived alone, $35 \%$ were male, $11 \%$ were long-term sick or disabled in regards to employment status, and $41 \%$ were classified as "clinically depressed." Furthermore, these older adults had 1.9 physical comorbidities, on average. Social isolation was divided into two categories: less than weekly direct contact with friends, family, and neighbors, and less than monthly direct contact with friends, family, and neighbors. Those who did not fit either category were deemed 'at risk for social isolation.' Health-related quality of life in these individuals was assessed using the SF-12 and the Euro Quality of Life (EQ-5D), which is a European instrument used to measure health-related quality of life. It consists of 5 dimensions, including mobility, self-care, usual activities, pain/discomfort, and anxiety/depression. There are 3 levels within each dimension, which ask the respondent to mark either no problems, some problems, or extreme problems (EuroQol Research Foundation, [EQRF], 2015). Scores from each of these tools were compared in the severely socially isolated, the socially isolated, and the 'at risk for social isolation' groups. Researchers discovered that $17 \%$ of participants were severely socially isolated, $24 \%$ were socially isolated, and $59 \%$ were at risk for social isolation. The number of adults in each social isolation category was also divided into age groups to compare to UK population age norms. Those age groups were $50-59,60-69,70-79$, and $>80$ years. The number of older adults who were categorized as 'at risk for social isolation' generally increased with the increasing age groups. However, there was no apparent trend between age groups for the older adults who were severely socially isolated. In addition, researchers conducted a regression analysis. After controlling for depression, physical co-morbidity, age, gender, living alone, accommodation type, and employment status, social isolation was significantly $(p<0.01)$ and independently associated with health-related quality of life 
scores. As the number of social contacts increased, the severity of social isolation decreased $\left(R^{2}=0.34\right)$. Those that were categorized as severely socially isolated also reported a health-related quality of life score 4.73 points lower than those who were not severely socially isolated, on average (Hawton et al., 2011).

Even though the study by Hawton et al. (2011) observed social isolation independent of depression, mental health tends to be associated with health status and quality of life in older adults (Gallegos-Carrillo et al., 2009). Depressive symptoms can be difficult to screen for, especially in older adults diagnosed with physical illness. Furthermore, older adults are less likely to report depressive symptoms compared to younger populations because of the negative stigma associated with mental illness (Tanner, Martinez, \& Harris, 2014). Mental health is also impacted by the number of chronic diseases older adults have (Gallegos-Carrillo et al., 2009). As adults age, the prevalence of multiple illnesses tends to increase, which may cause depressive symptoms and poor quality of life. Inability to perform ADLs are predictors of depression as well as quality of life in community-dwelling older adults (Tanner et al., 2014). ADLs are typically self-reported, and can reflect older adults' functional ability and mobility. Overall, the link between depression and functional status in community-dwelling older adults has rarely been studied (Gallegos-Carrillo et al., 2009). Moreover, the association between quality of life, mental health, and physical health in older adults is lacking research, especially in the U.S.

As described, quality of life is generally defined as overall life satisfaction, which includes a variety of dimensions that can affect a person's daily life and well-being (Amarantos et al., 2001). Quality of life tools can also be broadly used to assess groups of people in certain areas, and can therefore be analyzed for patterns in research studies and policy-making (CDC, 2011a). Health-related quality of life specifically focuses on mental and physical illness, and how these disease states can impact health 
and happiness (Drewnowski \& Evans, 2001). Both quality of life and health-related quality of life are measured subjectively using various tools, forms, and questionnaires. Each quality of life tool discussed earlier has its strengths, weaknesses, and limitations, and rarely has a component (measurement) dedicated to nutrition.

\subsection{Nutrition and Food in Relation to Quality of Life}

Various aspects of the food experience, such as the type of food people eat, what times of the day they eat, and how it is consumed, all play a role in quality of life, and should therefore be taken into account when assessing overall life satisfaction (Barr \& Schumacher, 2003a). This section describes the mealtime experience; research studies examining the relationship between adherence to diet, nutrition interventions, and quality of life; and nutrition-related quality of life tools proposed by researchers.

\subsubsection{The Mealtime Experience in Community-Dwelling Older Adults}

Food is an important aspect of daily life, and can either bring satisfaction or dissatisfaction depending on various factors involved (Grunert et al., 2007). The mealtime experience, for example, includes dietary habits, behaviors, taste, and social relationships (Amarantos et al., 2001). Mealtime is an important aspect of an older adult's routine because it provides a consistent structure, as well as something to look forward to on a daily basis (Mahadevan et al., 2014). Independent, community-dwelling older adults have the ability to make their own diet choices based on food preferences. Mealtime can provide a source of social interaction, which improves food intake and quality of life because of the enjoyment factor associated with eating with others. Societal norms involve women cooking and preparing food for their families, while men are expected to consume the food that is prepared for them. These traditional gender roles influence cultural norms and eating behaviors at mealtimes as well (Vesnaver \& Keller, 2011). Not only is the meal itself a factor affecting food experience, but there are 
also physiological, social, and environmental aspects that contribute to mealtime as a whole, which is why meal enjoyment should be considered with quality of life.

Certain factors can limit an older adult's ability to enjoy meals and quality of life. Some medications, for instance, can cause a change in the ability to chew, swallow, digest, and absorb vital nutrients. The aging process tends to slow down metabolism, which can result in a reduced appetite due to increased feelings of satiety. If older adults do not have the desire for certain foods, consuming all the nutrients needed on a daily basis may be impaired (Bernstein \& Munoz, 2012). Inadequate food and nutrient intake can lead to malnutrition, which can also lead to a poor health status, functional status, and overall health-related quality of life (Amarantos et al., 2001). Requiring feeding assistance from a caregiver or family member can also lead to a less enjoyable experience and can reduce quality of life due to the lack of independence and control over food choices (Bernstein \& Munoz, 2012). One functional disability that can negatively impact the pleasure of mealtime is dysphagia, or the inability to swallow effectively. Dysphagia occurs in $15 \%$ of community-dwellers and $40 \%$ of institutionalized older adults. Inability to swallow food safely can interfere with social interactions at mealtimes, thereby creating a poor eating experience for older adults (Humbert \& Robbins, 2008).

Food preferences may change with age, and the decline of the five senses may affect the types of foods older adults choose to consume. Lack of taste sensation is the most common, and can cause elders to add excessive amounts of sodium or sugar to previously healthy foods. Older adults may not be able to smell certain foods that were once appealing, which can alter appetite, willingness to cook, and eventually eating habits (Bernstein \& Munoz, 2012). These factors are also predictive of malnutrition, which can negatively affect quality of life due to these functional impairments as previously described (Rasheed \& Woods, 2013). A physical issue that may prevent older 
adults from preferring and consuming certain foods is oral health. Seniors tend to find certain fruits and vegetables unappealing because they may not have an adequate amount of strong teeth to chew food properly (Bernstein \& Munoz, 2012). These physiological and physical nutrition-related factors need to be taken into account when assessing quality of life in future research.

\subsubsection{Systematic Review, Observational, and Intervention Studies on Nutrition and Quality of Life}

Researchers Rasheed and Woods (2013) conducted a systematic review and meta-analysis in order to identify literature on nutritional status and its possible effects on quality of life in older adults. Both intervention and observational studies were included in the evaluation. The criteria for both types of studies included: intervention studies that measured quality of life before or after a nutritional intervention; intervention studies that compared quality of life in a nutritional intervention group and control cohort; and observational studies that compared quality of life in malnourished adults with controls. In addition, use of a validated quality of life tool and participants being aged 65 and older were required as part of the inclusion criteria. Individuals receiving medical treatment that could have an impact on nutritional status, such as chemotherapy and dialysis, were excluded. After the inclusion and exclusion criteria were taken into account, 30 studies were eligible for analysis, including 14 observational and 16 randomized control trials. The literature examined in this meta-analysis suggests that malnutrition can lower quality of life in older adults, with fifteen studies indicating an association between these two variables. In the intervention studies, participants were randomly assigned to receive either a nutrition intervention, which typically involved a nutrition supplement, or routine care serving as the control. Three of these trials did not contain enough data to determine the effects of a nutrition intervention on quality of life; however, the remaining thirteen studies indicated that the combined physical component 
of quality of life was significantly improved (standard mean difference 0.23 ) with a nutrition intervention, with a p-value $=0.002$ in the pooled analysis. Nine of these studies were able to show that a nutrition intervention can positively affect the mental aspects of quality of life as well (standard mean difference $0.24, p<0.001$ ). Characteristics of studies selected based on quality and relevance of the intervention and outcomes are described in detail in Table 2.3.

Overall, the pooled analysis indicated that quality of life was higher in wellnourished older adults compared to individuals with a poor nutritional status $(p<0.001)$. Most of these studies included high-risk older adults, including hospitalized elders, and a nutrition supplement improved their self-perceived HRQOL. Out of the 30 studies, 14 used the Short-Form 36 (SF-36), which is a commonly used health-related quality of life tool discussed in the previous section. Another tool used by the authors in this metaanalysis is the EQ-5D. General quality of life tools such as the SF-36 can be imprecise in assessing nutrition because there are no items directly linked to food-related activities and eating habits. If researchers did not use a quality of life tool with nutrition-related questions, the relationship between nutrition and quality of life may not have been properly measured (Rasheed \& Woods, 2013).

Carson and others (2014) also reviewed multiple studies that utilized a dietary intervention to assess changes in quality of life over time. Typically, the relationship between food intake and health is assessed using weight loss, lab values, and biomarkers. Because of this, Carson and colleagues aimed to identify the effectiveness of dietary interventions on quality of life, both independent and dependent of weight loss. The inclusion criteria were as follows: the study had quality of life as the outcome, there was a diet-related intervention, the study was intended to promote weight loss, the intervention period was at least 12 weeks long, the study involved participants at least 19 years of age, the publication was available in English with the publication date 
between January 1, 1990 and August 31, 2012, and the study was conducted in the U.S. Studies that provided surgery or medications for weight loss were excluded. 
Table 2.3 A Summary of 5 Studies Examining the Relationship Between Nutrition and Quality of Life

\begin{tabular}{|c|c|c|c|c|}
\hline Authors/Year & $\begin{array}{l}\text { Study Design and } \\
\text { Population }\end{array}$ & Intervention/Independent Variable & $\begin{array}{c}\text { Outcome/Dependent } \\
\text { Variable }\end{array}$ & Results \\
\hline $\begin{array}{l}\text { Payette et al., } \\
2002\end{array}$ & $\begin{array}{l}\text { Randomized } \\
\text { community trial. } \\
\text { Subjects recruited } \\
\text { from adults } 65+\text { at } \\
\text { high nutritional risk } \\
\text { receiving long-term } \\
\text { home help services } \\
\text { from community } \\
\text { service centers in } \\
\text { Canada. At baseline, } \\
\text { there were } 83 \\
\text { subjects; } 29 \% \text { were } \\
\text { men, average age } \\
\text { was } 80,65 \% \text { lived } \\
\text { alone, } 58 \% \text { practiced } \\
\text { regular light exercise } \\
\text { (>1/week), and the } \\
\text { average BMl was } \\
20.1 \mathrm{~kg} / \mathrm{m}^{2} \text { (=normal). } \\
43 \text { were randomized } \\
\text { to the experimental } \\
\text { group, } 46 \text { were } \\
\text { randomized to the } \\
\text { control group. }\end{array}$ & $\begin{array}{l}\text { Experimental group received two } \\
235-m L \text { cans per day of a liquid } \\
\text { nutrition supplement (Ensure) for } 16 \\
\text { weeks to supplement their overall } \\
\text { food intake. Control group did not } \\
\text { receive any treatment. }\end{array}$ & $\begin{array}{l}\text { Health and functional } \\
\text { status was assessed } \\
\text { using the SF- } 36 . \text { Scores } \\
\text { range from } 0 \text { (worst } \\
\text { health) to } 100 \text { (best } \\
\text { health). }\end{array}$ & $\begin{array}{l}83 \text { subjects completed the } \\
16 \text {-week study. From week } 0 \\
\text { to week } 16 \text {, the control group } \\
\text { had significantly improved } \\
\text { physical role functioning } \\
\text { scores ( } p<0.01 \text { ), and no } \\
\text { significant improvements in } \\
\text { emotional role functioning } \\
\text { and vitality scores. For the } \\
\text { compliant subjects in the } \\
\text { experimental group ( } n=23 \text { ), } \\
\text { physical role functioning, } \\
\text { emotional role functioning, } \\
\text { and vitality scores } \\
\text { significantly improved } \\
\text { ( } p<0.001, p<0.05, p<0.05 \text { ). } \\
\text { For all subjects in the } \\
\text { experimental group, physical } \\
\text { role functioning and } \\
\text { emotional role functioning } \\
\text { significantly improved } \\
\text { ( } p<0.01, p<0.01 \text { ). There } \\
\text { were no statistically } \\
\text { significant differences } \\
\text { between the experimental } \\
\text { and control groups. }\end{array}$ \\
\hline $\begin{array}{l}\text { Edington et } \\
\text { al., } 2004\end{array}$ & $\begin{array}{l}\text { Prospective } \\
\text { randomized } \\
\text { controlled trial, } \\
\text { conducted in } 4 \\
\text { hospitals in the UK. } \\
\text { Subjects being } \\
\text { discharged from the } \\
\text { hospital who were }\end{array}$ & $\begin{array}{l}\text { Experimental group received a } \\
\text { nutritional supplement (either Ensure } \\
\text { Plus, Enlive, Formance Pudding or } \\
\text { Ensure Bar) upon discharge and at } \\
\text { weeks } 4 \text { and } 8 \text {, which ranged from } \\
600-1000 \mathrm{kcal} / \text { day based on } \\
\text { patient's energy requirements } \\
\text { needed to gain } 0.5 \mathrm{~kg} / \text { week. The }\end{array}$ & $\begin{array}{l}\text { Quality of life was } \\
\text { assessed using the EQ- } \\
5 D \text {. }\end{array}$ & $\begin{array}{l}\text { At week } 24, \text { there were } \\
\text { statistically fewer mobility } \\
\text { problems in the intervention } \\
\text { group }(p=0.022) \text {. There were } \\
\text { no other statistically } \\
\text { significant differences in the } \\
\text { other domains, which were } \\
\text { self-care, usual activities, }\end{array}$ \\
\hline
\end{tabular}




\section{identified as}

malnourished were

included in the study.

At baseline, there

were 100 subjects

with a mean age of

$78.45 \%$ were male, $66 \%$ lived alone. 51

patients were

randomized to the

experimental group,

and 49 were

randomized to the

control group.
Hickson \&

Frost, 2004

Gariballa \&

Forster, 2007
Data was collected as part of a large randomized control

trial in the UK.

Subjects $(n=233)$

were sick, elderly in-

patients. $45 \%$ were

male, and the median age was 81 .

Randomized, doubleblind, placebocontrolled trial of hospitalized elderly in the UK. At baseline $(n=225)$, mean age was $75,63 \%$ were male, $31 \%$ never smoked, $61 \%$ owned their home, and the total average SF-36 score was 82 . One-

hundred six were

randomized to the control group did not receive any

Dietitians visited the subjects at

home at weeks $4,8,12$, and 24 to

measure weight, and calculate BMI.

Nutritional status

Quality of life, assessed using the EQ-5D

The experimental group received two bottles $(200 \mathrm{~mL}$ each) of an oral nutritional supplement at 8:00am and 12:00pm daily in addition to the standard hospital diet for 6 weeks.

Supplement was $995 \mathrm{kcal}$. The control group received a placebo, which was a supplement identical to the treatment but contained no protein or micronutrients and provided $60 \mathrm{kcal}$.
Quality of life was assessed using the SF36. supplements upon discharge. pain/discomfort,

anxiety/depression in either group.
Subjects were split into two subgroups: $65-74$ and $75+$ years of age. Both age groups had a significantly lower EQ-5D index score compared to free living elderly in the UK $(p<0.001$ for both).

Quality of life was measured at baseline (week 0), 6 weeks, and at week 24 (endpoint). At 6 weeks, the mean difference in overall

QOL scores between the placebo and supplement groups was not statistically significant $(1.8, p=0.86)$.

After 6 months, the mean difference was statistically significant $(-8.7, p=0.003)$.

However, difference in cumulative change between 
experimental group,

and 119 were

randomized to the

control group.

Johansson et

al, 2009

Prospective

longitudinal study

conducted in

Sweden. 482

community-dwelling

older adults aged

$75+$ were selected

from a national

register to participate

in the study.

the two groups was not significant $(p=0.08)$.

Nutritional status was assessed

using the Mini Nutritional

Assessment (MNA). Results of the

MNA were categorized as 'well

nourished' (24-30 points) and 'at risk

for malnutrition' (<24 points).
Quality of life was assessed using the Nottingham Health Profile (NHP). Scores for each domain range from 0 (no problems) to 100 (all problems)
Domains for the NHP include physical mobility, pain,

sleep, energy, social

isolation, and emotional

reactions. A higher score indicates a greater number and severity of problems.

Women at risk for

malnutrition $(n=52)$ reported

significantly higher NHP scores for each domain

(physical mobility, pain energy, and emotional

reactions had $\mathrm{p}$ -

values $<0.001$, sleep had a $p$ value $=0.041$, and social isolation had a pvalue $=0.047$ ) compared to women at no risk for malnutrition $(n=225)$. Men at risk for malnutrition $(n=32)$ reported significantly higher

NHP scores for physical mobility $(p=0.018)$, sleep $(p=0.003)$, energy $(p=0.004)$, and social isolation $(p=0.024)$ domains

compared to men at no risk for malnutrition.

Table layout adapted from Rasheed \& Woods (2013). Study design and results sections of table were summarized from the primary research articles. 
The most common intervention was a calorie-restricted diet. Overall, $88 \%$ of the studies reviewed reported improvements in quality of life with the weight loss intervention. However, the researchers in $50 \%$ of those studies did not clarify whether the improved quality of life score was due to the dietary intervention directly or if it was just a result of weight loss. Furthermore, the myriad of factors surrounding dietary intake and quality of life, such as social interactions, satiety, economics, physical aspects, and psychological aspects, make it difficult to determine a linear relationship. Therefore, the direct link between nutrition and quality of life needs to be addressed in future research studies (Carson et al., 2014).

Germain and colleagues (2013) followed a French cohort consisting of 12,741 participants for 12 years to determine the relationship between nutrition compliance with dietary guidelines and health-related quality of life. Nutrition data were obtained through 24-hour diet recalls, which were completed by the participants every two months. Subjects received a manual in order to properly record food portions. Compliance with dietary guidelines was scored based on deviation from recommendations of each food group. Scores typically ranged from -0.5 to 2 , with higher scores indicative of higher adherence to guidelines. Health-related quality of life was assessed using the SF-36 at baseline and at the conclusion of the study. Scores range from 0 to 100 , and higher scores in each quality of life domain indicate a higher functional status. There were 3,005 subjects included in the final analysis, with an age range of $45-60$ years and a mean age of 51 years at baseline. Fifty-four percent of the subjects were male, and $46 \%$ were female. Seventy-eight percent of subjects were employed, and $13 \%$ lived alone. From baseline to the 12-year endpoint, the health-related quality of life scores that significantly decreased were physical functioning, bodily pain, and general health $(p<0.0001)$. Mental health, emotional role, social functioning, and vitality significantly increased $(p<0.0001)$, and there was no significant change in the physical role category 
$(p=0.38)$. According to the final results, dietary compliance was associated with a high physical component summary score, which was an aggregated score of the quality of life dimensions related to physical health (i.e. physical functioning, role-physical, bodily pain, and general health). Specifically, those who adhered to French dietary guidelines had a positive change in the physical components of health-related quality of life over 12 years $(p=0.0478)$, but not in the mental components $(p=0.4930)$, which included vitality, social functioning, role-emotional, and mental health. However, mental health-related quality of life was related to dietary compliance initially $(p=0.0011)$, but did not significantly change throughout the duration of the study (Germain et al., 2013). Overall, the results suggest that eating behaviors may affect the physical dimensions of health-related quality of life over the course of 12 years, but not the mental dimensions.

Similarly, Gopinath and colleagues (2014) observed the relationship between adherence to dietary guidelines and quality of life by following a large cohort in Australia for 15 years. At baseline, there were 1,305 participants aged 49 and older with mean age 67, who were recruited from the Blue Mountains Eye Study (BMES). Forty-one percent of the participants were male, $24 \%$ lived alone, $94 \%$ owned a house, and $5 \%$ had a walking disability. Participants' nutritional information was acquired using a 145item food frequency questionnaire (FFQ) at baseline, and after 5, 10, and 15 years. The Australian Diet Quality Index and the Australian Guide to Healthy Eating were used to determine the total diet score, based on the FFQ responses. The total diet score is indicative of adherence to the Dietary Guidelines for Australian Adults (DGAA). Total diet scores can range from 0 to 20 , where higher scores indicate higher compliance with dietary guidelines. As seen in the French cohort study, the SF-36 was also utilized in this study to assess quality of life after 5 and 10 years. At the conclusion of the study, the total diet scores were separated into quartiles, in which the lowest quartile was the lowest group of diet scores. Each domain of the SF-36 was compared with the total diet 
scores. Over the course of five years, there was a statistically significant difference between the diet score quartiles in relation to the following quality of life domains: physical functioning, role limitations due to physical problems, general health, vitality, and overall physical composite score, as shown in Table 2.4.

Compared with the lowest diet score quartile, the highest quartile had higher quality of life scores, indicating an association between these two variables. Data was not significant for the other quality of life domains, which included bodily pain, social function, role-emotional, mental health, and the mental composite score (Gopinath et al., 2014). Results suggest that some aspects of quality of life, which mainly include the physical domains, could be improved by improving diet quality in older adults. The large sample size, time frame, and use of validated tools to collect dietary and quality of life data increased the strength of the study. One possible weakness was the use of a FFQ because it is difficult to "average" all foods consumed in one year. Remembering portion sizes and amounts of each food group may also have been an issue, especially for older adults. 
Table 2.4 Statistically Significant Associations Between Total Diet Scores and Quality of Life Scores by Domain After 5 Years

\begin{tabular}{|c|c|c|c|c|c|}
\hline $\begin{array}{l}\text { Quality of } \\
\text { life domain }\end{array}$ & $\begin{array}{c}1^{\text {st }} \text { Quartile } \\
\text { Total Diet } \\
\text { Score } \\
<8.13\end{array}$ & $\begin{array}{c}2^{\text {nd }} \text { Quartile } \\
\text { Total Diet } \\
\text { Score 8.15- } \\
9.75\end{array}$ & $\begin{array}{c}3^{\text {rd }} \text { Quartile } \\
\text { Total Diet } \\
\text { Score 9.76- } \\
11.10\end{array}$ & $\begin{array}{c}4^{\text {th }} \text { Quartile } \\
\text { Total Diet } \\
\text { Score } \\
>11.13\end{array}$ & $P$ for trend \\
\hline $\begin{array}{l}\text { Physical } \\
\text { functioning }\end{array}$ & $66.0^{a}$ & 70.7 & 70.1 & 71.6 & 0.003 \\
\hline $\begin{array}{l}\text { Role- } \\
\text { physical }\end{array}$ & 57.6 & 60.7 & 62.9 & 63.7 & 0.05 \\
\hline $\begin{array}{l}\text { General } \\
\text { health }\end{array}$ & 65.2 & 66.2 & 66.7 & 69.2 & 0.02 \\
\hline Vitality & 57.0 & 60.4 & 61.3 & 62.3 & 0.001 \\
\hline $\begin{array}{l}\text { Physical } \\
\text { composite } \\
\text { score }\end{array}$ & 42.4 & 42.9 & 43.2 & 45.0 & 0.003 \\
\hline
\end{tabular}

${ }^{a}$ Mean quality of life scores, adjusted for age, sex, receipt of pension payment, home ownership, hospital admission, walking disability, having 5 or more chronic conditions, cognitive impairment, visual impairment, and living alone.

Data from Gopinath et al., 2014.

Researchers created a Healthy Aging and Happy Aging (HAHA) program in Korea that integrated health education and physical activity for community-dwelling older adults with hypertension. Program effectiveness was studied in order to determine if there was a positive influence on hypertension control, exercise, and health-related quality of life. To be eligible to participate, the individuals had to be 65 or older, attend a senior center frequently, and be diagnosed with hypertension for at least 1 year. After the inclusion criteria were specified, 45 of the 80 recruited older adults were eligible. Twenty-two were randomly assigned to the experimental group, and 23 were in the control group. The HAHA program was 12 weeks long, and included health education in a group setting once a week, individual health counseling at week four, and an elastic band exercise class twice a week. The first five weeks were dedicated to educating the 
participants on hypertension, which included information on symptoms, medications, and how to check and interpret blood pressure readings. The health topics from week 6 to week 12 consisted of diet, exercise, stress management, emergency care, smoking and alcohol, self-management strategy, and final program evaluation. Just like the two cohort studies described above, health-related quality of life was assessed using the SF-36. Results indicated that the general health, vitality, social functioning, and mental health scores significantly improved in the intervention group and not in the control group from baseline to week 12 . There was no difference between the intervention and control groups for physical functioning, role-physical, bodily pain, and role-emotional. Using a randomized controlled trial strengthened this study because it is the best way to determine a possible causal relationship between two variables. Also, using an individualized exercise routine and counseling session appeared to be beneficial, suggested by the $81.8 \%$ retention rate. The sample size was sufficient to detect an effect size with $80 \%$ power at an alpha level of 0.05 (Park et al., 2010).

Assaf and colleagues (2016) evaluated the effect of the Women's Health Initiative (WHI) Dietary Modification (DM) intervention on HRQOL in 48,835 women aged 50-79 years old. The women were recruited from 40 clinical centers throughout the US. The goal of the WHI DM intervention was to reduce fat intake to $20 \%$ of total calories based on the women's height, increase fruit and vegetable consumption to 5 or more servings per day, and increase grain consumption to 6 or more servings per day. Trained nutritionists held 18 group sessions throughout the one-year intervention period that provided information and activities regarding the DM intervention. Quality of life was assessed using the SF-36, along with a global QOL item asking, "Overall, how would you rate your quality of life?" This one global QOL item was on an 11-point scale, with $0=$ "As bad or worse than being dead" and $10=$ "Best quality of life." After one year, the SF-36 component scores for general health, physical functioning, and vitality significantly 
improved with the DM intervention, after adjusting for age, ethnicity, and weight, suggesting that dietary modification is associated with improved HRQOL (Assaf et al., 2016).

\subsubsection{Proposed Nutrition-Related Quality of Life Tools}

Generic quality of life and health-related quality of life tools, such as the SF-36, do not contain elements directly related to nutrition (Amarantos et al., 2001). The SF-36 has a physical functioning dimension that includes questions regarding the ability to lift and carry groceries, but no dimensions and items directly asking about nutrition and food-related activities (Ware, 2000). Therefore, quality of life tools directly involving nutrition domains and items have been proposed by a few research groups over time. Corle and colleagues (2001), for example, created and utilized a quality of life tool specifically for their nutrition-related study. Sponsored by the National Cancer Institute $(\mathrm{NCl})$, researchers conducted a Polyp Prevention Trial (PPT) in order to investigate the effect of a low-fat, high-fiber, and high-fruit and vegetable diet on the frequency of polyps in the large intestine. Quality of life over the course of 4 years was also assessed in regards to the individual's eating habits and disease state. There were 394 participants that were randomly assigned to either the intervention or control group who had large bowel polyps removed within 6 months of the study. At baseline, the average age was 62 years old, $80 \%$ were married, $67 \%$ received more than a high school education, $62 \%$ were men, $11 \%$ smoked, $11 \%$ were from minority populations (either African American, Hispanic, Indian/Native American, Asians/Pacific Islanders, and others) and the average BMI was 27.5 , which is categorized as overweight. There were no statistically significant differences between the intervention and control groups at baseline. The intervention group ( $n=194$ ) received nutrition counseling in order to obtain a diet with $20 \%$ energy from fat, $18 \mathrm{~g}$ of fiber per $1,000 \mathrm{kcal}$, and 3.5 servings of fruits and vegetables per 1,000 kcal. The control group $(n=200)$ received no nutrition counseling and was asked to 
maintain their usual eating habits. The quality of life tool created to assess diet was the Quality of Life Factors (QF) Questionnaire, which contains 9 domains and 51 items derived from other questionnaires and health-related models. Scores were based off a 3 or 4-point Likert scale. The domains and number of items in each, selected items, and responses for each type of Likert scale are shown in Table 2.5.

Table 2.5 Quality of Life Factors (QF) Questionnaire Domains, Number of Items, Selected Items, and Likert-Scale Response Type

\begin{tabular}{|c|c|c|}
\hline Domains & $\begin{array}{l}\text { Number of } \\
\text { Items }\end{array}$ & Example Items and Response Type \\
\hline Social & 11 & $\begin{array}{l}\text { I enjoy the foods I eat at holiday meals and parties } \\
\text { Disagree a lot, Disagree a little, Agree a little, Agree a lot }\end{array}$ \\
\hline Self-care & 8 & $\begin{array}{l}\text { Eating the way I do now makes me feel good about myself } \\
\text { Disagree a lot, Disagree a little, Agree a little, Agree a lot }\end{array}$ \\
\hline $\begin{array}{l}\text { Health } \\
\text { assessment }\end{array}$ & 7 & $\begin{array}{l}\text { During the past month, how have you been feeling generally? } \\
\text { Poor spirits, Fairly good spirits, Good spirits, Very good spirits }\end{array}$ \\
\hline Convenience & 5 & $\begin{array}{l}\text { Is it hard or easy to shop for the kinds of food you or your family } \\
\text { eat? } \\
\text { Very hard, A little hard or don't know, Pretty easy, Very easy }\end{array}$ \\
\hline Cost & 5 & $\begin{array}{l}\text { Sometimes it's hard for me to pay for the kind of food I eat } \\
\text { Disagree a lot, Disagree a little, Agree a little, Agree a lot }\end{array}$ \\
\hline Taste & 4 & $\begin{array}{l}\text { I am satisfied with the taste of food I eat } \\
\text { Disagree a lot, Disagree a little, Agree a little, Agree a lot }\end{array}$ \\
\hline Health action & 4 & $\begin{array}{l}\text { During the past month, how often have you received nutrition or } \\
\text { health messages from the following sources: media, food labels } \\
\text { Never, Sometimes, Often }\end{array}$ \\
\hline Life satisfaction & 4 & $\begin{array}{l}\text { In general, how satisfying is your life? } \\
\text { Very unsatisfying, Fairly unsatisfying, Fairly satisfying, Very } \\
\text { satisfying }\end{array}$ \\
\hline Health Belief & 3 & $\begin{array}{l}\text { How likely is it that food choices you can make will: improve } \\
\text { your health? Help you feel better? } \\
\text { Very unlikely, Somewhat unlikely, Somewhat likely, Very likely }\end{array}$ \\
\hline
\end{tabular}

Adapted from Corle et al (2001).

The QF Questionnaire was put together based off of a variety of quality of life assessment tools and health-related models. All of the items in the self-care, convenience, cost, and taste domains were derived from the Southeast Cholesterol Project QOL Questionnaire. Four items in the health assessment domain came from the 
SF-36, two came from the Health Locus of Control Questionnaire, and one was from the Psychological General Well-Being Scale. All four questions in the life satisfaction domain came from Bryan and Veroff's QOL measure. Questions in the health belief and health action domains were based on Becker's Health Belief Model (Corle et al., 2001).

Participants were asked to complete the QF Questionnaire at baseline and annually for the duration of the study, which was 4 years. Cronbach's alpha was used to measure the internal consistency of these domains and reliability for the items ranged from 0.5 to 0.9 at baseline. A modified FFQ for the PPT was also completed at baseline and annually with the QF Questionnaire, which added more low-fat, nonfat, and high fiber foods. The items in the QF Questionnaire were standardized to have a score between 0 and 1, indicating worst quality of life to best quality of life. The total score for each domain was determined by adding up all the responses and multiplying by 100 to have a score between 0 and 100, again, indicating worst quality of life to best quality of life. Overall, the QF Questionnaire had some items directly related to nutrition, but is still considered a broad quality of life tool. In addition, internal consistency testing at baseline was the only reliability test mentioned in the study. Further validity and test-retest reliability testing is needed to develop a nutrition-related quality of life tool that contains more domains of food-related life (Corle et al., 2001).

Barr and Schumacher (2003b) documented a step-by-step process to develop a Nutrition Quality of Life (NQOL) survey that could be used in the field of dietetics, which was proposed by them five months prior (Barr \& Schumacher, 2003b). The purpose of this tool is to help determine the effect Medical Nutrition Therapy (MNT) has on patients' quality of life. Because MNT is typically utilized in an outpatient setting, Barr and Schumacher proposed that the NQOL survey should not take longer than 10 minutes to fill out, and should be at a $5^{\text {th }}$ grade or higher reading level. The first step in developing this NQOL survey was brainstorming, which involved focus group sessions with 111 
patients who have received MNT within the last 6 months to identify possible items and questions that could be included in this survey. The sessions were held in Boston, Chicago, and Phoenix in order to gain feedback from clients and dietetic professionals in a variety of geographic locations. The American Dietetic Association (ADA) helped identify Registered Dietitians (RDs) in these areas that were willing to facilitate the focus groups. After the four sessions were held, the researchers took everyone's ideas into account before creating a set of questions related to MNT and QOL. Step two also consisted of focus groups. For this step, geographic locations included two locations in Arizona, two locations in California, Boston, Chicago, and Nashville. Again, RDs near each site were asked to facilitate the group sessions. For the patient focus groups, the RDs were asked to recruit six to eight individuals per group with different backgrounds and demographics for diversity. For dietetics professionals focus groups, the RDs were asked to recruit professionals from various practices in the area. Proposed questions were discussed among each focus group, which lasted up to 90 minutes. Step three was item development, in which researchers studied the feedback from the brainstorming and focus group sessions to create the first draft of the NQOL tool, with assistance from dietetic consultants. Based on current literature regarding QOL measurement tools and common themes identified throughout the focus groups, a framework involving categories and items within them was created as NQOL version 1.1. In step 4, the patients and RDs who were a part of the previous focus groups were asked to take the NQOL version 1.1. The survey was mailed out, and participants were asked to anonymously complete the survey, record how long it took to complete it, and answer questions about the survey at the end with any additional comments and suggestions. Fifty-seven percent of patients and $67 \%$ of dietetics professionals completed the questionnaire, which took an average of 9.8 minutes. Once the surveys were received, the item development team looked at each comment and suggestion, and modified the 
survey as necessary. This resulted in NQOL version 1.2. In the fifth step, new patients and dietetics professionals were asked to participate in focus groups. This version of the NQOL was also presented at the Annual Meeting of the ADA to receive additional feedback. After revisions were made, NQOL version 1.3 was created. Step six was the last round involving suggestions for improvement. People who attended the last plenary session at Nutrition Week 2002 were presented with NQOL version 1.3, and were asked to make comments directly on the survey or by contacting the researchers at a later time. Once modifications were made based on participants' comments, the NQOL version 1.4 was created. The final product had 50 items separated into 6 categories, which included food impact (items 1-9), self-image (items 10-15), psychological factors (items 16-25), social/interpersonal items (items 26-32), physical (items 33-41), and selfefficacy (items 42-50). The 50 items are listed in Appendix F.

Grunert and colleagues (2007) proposed a satisfaction with food-related-life measure that would consist of a quality of life domain strictly related to food. The researchers define food-related life as all aspects of dietary habits and needs, including grocery shopping, preparation, and consumption. The seven items suggested for this type of measure are shown in Table 2.6. These items are prefaced with a statement asking the participants to think of all food-related activities, such as planning, shopping, and preparing for meals to prepare them for the questions, along with a Likert scale from 1-7 to indicate their agreement with each statement. Data from this study was collected for the Food in Later Life project, which is designed to improve food-related quality of life in European older adults (Grunert et al., 2007). 
Table 2.6 Proposed Items for Measuring Satisfaction with Food-Related Life

Proposed Items

Food and meals are positive elements in my life

When I think of my next meal, I only see problems, obstacles, and disappointments

I am generally pleased with food

Food and meals give me satisfaction in daily life

My life in relation to food and meals is close to my ideal

I wish my meals were a much more pleasant part of my life

With regard to food, the conditions of my life are excellent

Adapted from Grunert et al., (2007).

Eight European countries participated in this study, which included Denmark, Germany, Italy, Poland, Portugal, Spain, Sweden, and the United Kingdom. Overall, 3 studies were conducted among these countries to test the proposed items described in Table 2.6. The first study was conducted from 2003-2004, with 96-105 older adults aged 65+ from each of the 8 countries, for a total of 760 individuals living at home. Participants were grouped by age, which included younger seniors aged 65-75 and older seniors aged 75 and above. Older adults were also grouped according to their living situation, which was either living alone or living with others. Results from this first study were utilized to determine any adjustments the scale questions needed, and to assess reliability and construct validity when compared to the SF-36 and Nutritional Health Index (NHI). The second study, conducted 6 months after study 1, involved 644 older adults. Of these, 459 also participated in the first study, allowing test-retest reliability to be assessed. Convergent validity was also evaluated by relating the satisfaction with food-related life 
scale (SWFL) to other measures of satisfaction with food. The first measure was an indepth interview, which typically lasted an hour long and included questions related to the preparation, production, and importance of meals. The second measure was similar to the SWFL but was over a short period of time. The older adults kept a 1-week food diary, and were then asked their satisfaction with life in relation to food after that one week. However, the item "when I think of my next meal" was removed when testing for internal consistency because it was the only statement that was time-specific. The other 6 items do not have a time frame associated with them. The third study, conducted in 2005 , had 3,291 new older adults aged 65+ from the 8 European countries. The participants were grouped based on age and the living situation like the previous two studies, and construct validity was assessed by comparing quality of life measures between the SWFL, SF-8, and the Satisfaction with Life Scale. A satisfaction with food-related life measure such as the one proposed by these researchers would be beneficial in many ways. These items could be used to determine possible relationships between food and lifestyle, and how changes in these can affect overall life satisfaction. It could be used on an international level as well to analyze possible differences between countries. Furthermore, older adults who have issues carrying out food-related activities could be identified, and satisfaction with food-related life could be improved as a result (Grunert et al., 2007).

Schunemann and others (2010) also proposed a generic quality of life tool specifically related to food and nutrition. Eighty-one participants, with an average age of 48 , were recruited in Italy to assess the initial tool in order to determine if the number of items needed to be reduced. Originally, 187 items were created based on interviews, advice from nutrition experts, and current literature on the subject. After careful consideration and pretesting, the quality of life tool was reduced to 29 items, making it much more manageable for participants to respond to since it takes around 12 minutes 
to complete. These nutrition-related quality of life items were subcategories under 5 main domains, which included healthy lifestyle, symptoms, sensations, social and role function, and taste. The questionnaire associated with these domains has questions asking how the participants felt in the last 4 weeks in relation to food intake. Examples include heartburn, sleepiness, satisfaction, and tranquility. The entire list of items, which had to be translated from Italian to English, is shown in Table 2.7. Since the original tool was in Italian and the translation may have altered some of the original wording, it is difficult to use this tool in different cultures and languages, but the general premise of each question is still present. Each dimension is related to nutrition and quality of life, which is rarely seen in general quality of life tools. Researchers used a Likert scale to score these items, which asked participants to rank importance, agreement, and frequency of occurrence on a scale from 1-7. The questionnaire was validated in 3 ways. First, 2,576 participants were recruited during a new food product presentation in temporary shops in Milan and Rome. Individuals were prompted with the purpose of the study and completed the questionnaire on a computer. The next recruitment process involved 128 individuals who were recruited through online advertising. These participants also completed the SF-36 online. Lastly, 20 individuals were recruited for reliability testing and were asked to complete the questionnaire two times, four weeks apart. Both convergent and discriminant cross-sectional construct validity were assessed by calculating Pearson's correlation coefficients. The 5 domains utilized in this study were compared with the 8 domains of the SF-36, and the correlation coefficients ranged from -0.078 to 0.562 . There were 40 correlations total, and 19 were statistically significant $(p<0.05)$. Domain-domain correlations were also calculated, which ranged from 0.141 to 0.456 . All of the $p$-values for these correlations were statistically significant $(p<0.001)$. 
Table 2.7 Items in Schunemann and Others' Nutrition-Related Quality of Life Tool Items

1. Warned heaviness

2. Warned acidity/heartburn stomach

3. Avoided heavy foods/fatty/fried

4. Experienced drowsiness

5. Warned satisfaction/morale relief

6. Avoided large quantities of food

7. Warned moment tranquility

8. Ate new dish

9. Intestinal disorders

10. Controlled labels/type of food eaten

11. Opportunity to meet

12. Have been shopping/participated in the preparation of meal/respected seasonal food

13. Eating food with tastes who does not like

14. Avoided going to sleep after eating/made a walk

15. Warned swelling

16. Below power supply which includes all the food groups

17. Eat healthy food

18. Eating food with good taste

19. Warned wellness/personal pleasure

20. Warned sensation recovery forces

21. Agreement on food tastes/dinner well cooked improved relationship with partner/family

22. Happy after abundant breakfast

23. Eating foods that prevent disease

24. Warned satiety

25. Controlled the intake of foods that make you fat

26. Consumed quality products

27. Smelled a dish 
28. The time to socialized/talk

29. Felt bad mood in relation to a meal

Adapted from Schunemann and colleagues (2010).

One weakness is that the tool may not be applicable to the general population because it was only tested in Italy, and requires translation before being utilized in other countries (Schunemann et al., 2010). Overall, this tool could mainly be used to determine if nutrition interventions are effective in influencing quality of life outcomes, but further testing to determine longitudinal construct validity is needed before establishing this instrument.

\subsection{Summary, Research Needs, and Thesis Research Questions}

Quality of life is an important outcome measure because it utilizes subjective evaluations which are predictors of morbidity and mortality (CDC, 2012). In fact, Healthy People 2020 made quality of life one of the four overarching public health goals because of its importance to daily life (USDHHS, 2014). Not only is overall quality of life affected by mental, physical, and social aspects of life, but food and nutrition may have an impact as well. A poor diet, which usually includes low amounts of whole grains, fruits and vegetables, increases risk of developing chronic diseases such as diabetes and cardiovascular disease, which can lead to poor quality of life as a result. Furthermore, malnourishment can impair physical functioning. Those that are malnourished, especially older adults, may need help with eating because of poor functional status, and that loss of control and independence may also contribute to a lower quality of life. However, future research is necessary in several aspects of nutrition and quality of life in older adults. Specifically, more work is needed to expand QOL and HRQOL to include food and meal-related domains. Even with the current HRQOL measures, little is known about diet and nutrition related to HRQOL, especially in community-dwelling older adults. Therefore, this thesis used the cross-sectional dataset National Health and Nutrition 
Examination Survey (NHANES) in order to investigate the relationship between nutrition and HRQOL in a nationally representative sample of older adults aged 60 and above. The broad thesis questions and their hypotheses are described below.

1. What is the relationship between key nutrition-related factors (especially diet quality) and overall $\mathrm{HRQOL}$ in older adults aged $60+$ ?

Hypothesis: A higher quality diet is associated with better HRQOL, after controlling for other important diet/nutrition variables (government meals delivered, meals eaten at a senior center, poor appetite/overeating and difficulty with meal prep), and key demographics (age, gender, race, education, marital status, income, and living alone).

2. What is the relationship between key nutrition-related factors (especially diet quality) and healthy days measures in older adults aged $60+$ ?

Hypothesis: A higher quality diet is associated with fewer unhealthy days, after controlling for other important diet/nutrition variables (government meals delivered, meals eaten at a senior center, poor appetite/overeating, difficulty with meal prep), and key demographics (age, gender, race, education, marital status, income, and living alone). 


\section{Chapter 3: Materials and Methods}

This section describes the specific NHANES datasets and study sample used for this thesis; the predictor and outcome variables selected, and the methods for selection; and the statistical models developed for addressing the main research questions.

\subsection{NHANES Background, Design, and Operation}

The National Health and Nutrition Examination Survey (NHANES) is a multistage, cross-sectional survey designed to be representative of the noninstitutionalized US population. As a major program of the National Center for Health Statistics (NCHS), NHANES combines both interview and physical examination components in order to assess the health and nutritional status in US children and adults. Demographic, socioeconomic, diet, and health information is collected through interviews, while medical, physiological, and laboratory tests are obtained through physical examinations in the Mobile Examination Center (MEC). Approximately 5,000 participants from 15 counties across the US are included in the survey each year. Data are released in 2-year cycles; as explained later, data from 2009-2010 and 2011-2012 were used for this thesis research. Findings from NHANES are used to set national standards for height, weight, and blood pressure measurements, to assess food and nutrient intakes, and to identify risk factors and prevalence of diseases, in addition to assessment of nutritional status and its association with health (CDC, 2014a).

NHANES utilizes a complex, 4-stage probability sampling design to obtain a random sample of adults and children across the US. In the first stage, Primary Sampling Units (PSU's) are selected, which are usually individual counties. Segments within those counties are selected in stage two. The third stage consists of choosing Dwelling Units (DUs) or households within the segments, and individuals within those households are selected in the last stage (CDC, 2013a). Once these individuals are 
chosen, participants are assigned a sample weight to account for multiple aspects of the NHANES survey design, including survey non-response, probability of selection, and differences between the sample and total population. Each sample weight is a measure of the number of people represented by that specific sample person, thereby making the weighted data representative of the entire non-institutionalized US population. Sample weights allow estimation of statistics that would have been obtained if the entire sampling frame had been chosen to participate, and are adjusted for nonresponse for both the in-home interview and the MEC examination. The NHANES protocol was approved by the NCHS Ethics Review Board (ERB), and all participants or their proxies gave informed consent (CDC, 2013b). In addition, the Cal Poly Human Subjects Committee reviewed this thesis research and deemed it exempt from further review because it is a secondary analysis of a publicly available dataset.

In order to increase reliability and precision of NHANES data, oversampling of certain population subgroups is conducted. In the $2009-2010$ survey years, for example, the following populations were oversampled: Hispanics, non-Hispanic blacks, lowincome non-Hispanic white and other persons at or below $130 \%$ of the federal poverty level, and non-Hispanic white and other persons aged 80 and over (CDC, 2013c). The 2011-2012 survey cycle also oversampled non-Hispanic Asian persons in addition to the same groups oversampled in the 2009-2010 survey cycle. An additional race/Hispanic origin variable, RIDRETH3, was created to account for this oversampling of Asians for years 2011-2012. The variable RIDRETH1, included in both 2-year survey cycles, consists of the categories "Mexican American," "Other Hispanic," "Non-Hispanic White," "Non-Hispanic Black," and "Other Race-Including Multi-Racial." RIDRETH3 includes "Non-Hispanic Asian" in addition to the RIDRETH1 categories. In the 2009-2010 dataset, Asians were part of the "Other" category in the RIDRETH1 variable. Because RIDRETH1 and RIDRETH3 are separate variables with different code values, the 
variables cannot be combined across the survey cycles (CDC, 2013a). Therefore, to use comparable race/Hispanic origin categories from 2009-2012, RIDRETH1 must be used. The following section describes the specific sample of older adults examined for this thesis and the rationale behind choosing it.

\subsection{Study Sample}

NHANES datasets are released to the public in 2-year cycles. For this research, data regarding dietary factors and health-related quality of life were analyzed using the 2009-2010 and 2011-2012 datasets combined (CDC, 2013a) to obtain a larger older adult sample size. For this thesis, dietary data in the most recent NHANES cycle (20132014) was not yet released; therefore, the two most recent 2-year datasets that had all dietary, questionnaire, and demographic data available were combined and analyzed. Since NHANES includes questions about senior food programs with a target population aged 60 and above, the older adult age group was defined as $60+$ for this particular data analysis. Moreover, senior nutrition programs such as the Elderly Nutrition Program (ENP), Supplemental Nutrition Assistance Program (SNAP), and the Commodity Supplemental Food Program (CSFP) define an older adult as 60 or older (Millen et al., 2002; USDAFNS, 2014a; USDAFNS, 2014b), further justifying the choice in age range for this thesis. According to the Administration on Aging (AOA), an older adult is defined as $65+($ ODPHP, 2014b; USDHHS, 2015), but for statistical purposes the age range begins at 60 years in order to categorize older adults by age groups $60-69$ years, $70-79$ years, and $80+$ years. Ages of survey participants are reported from 1-79 years. Older adults aged 80 and older are top coded as 80 in order to retain anonymity (CDC, 2015a).

\subsection{Variables Selection}

This section describes the NHANES variables selected for this thesis and the rationale behind those choices. The main outcome variables and predictor variables are 
described along with the preliminary analyses conducted on these variables to determine which were most appropriate for inclusion in the regression models.

\subsubsection{Health-Related Quality of Life Outcome Variables}

Health-related quality of life (HRQOL) is an important aspect of national health surveys such as NHANES. It is a valid indicator of the health of the nation, and can contribute to policy changes, community interventions, and achievement of national health objectives as stated by Healthy People 2020 (CDC, 2011a), yet little is known about the predictors of HRQOL in older adults. For these reasons, HRQOL was selected as the outcome measure in order to determine its association with diet-related and other predictor variables. The next section describes how HRQOL is measured in NHANES and how it was used for this research.

To measure health-related quality of life in a population, the CDC uses their set of questions called CDC HRQOL-14 "Healthy Days Measures." The three modules within these healthy days measures are the Healthy Days Core Module, the Activity Limitations Module, and the Healthy Days Symptoms Module, which have 14 questions total (CDC, 2012), as shown in Appendices C, D, and E. At a national level, CDCHRQOL measures can be used to identify health status indicators and potential differences between populations across the US, and to track population trends over time (CDC, 2011b). The Healthy Days Measures are also used to track success with Healthy People 2020 goals, which provide 10-year national objectives for Americans to follow in order to improve their health and well-being (ODPHP, 2015). The Healthy Days Core Module that contains four questions regarding HRQOL has been used in NHANES since the year 2000 (CDC, 2012), and these variables were used as the outcome variables for this research.

The CDC Healthy Days Core Module questions are in the NHANES Current Health Status Questionnaire, which contains interview data regarding quality of life and 
recent illnesses from questions asked to participants aged 12 and up. Since healthrelated quality of life was the outcome for this thesis, these four variables were chosen: HSD010: Would you say your health in general is...?

HSQ470: The next questions are about your recent health during the 30 days outlined on the calendar. Thinking about your physical health, which includes physical illness and injury, for how many days during the past 30 days was your physical health not good?

HSQ480: Now thinking about your mental health, which includes stress, depression, and problems with emotions, for how many days during the past 30 days was your mental health not good?

HSQ490: During the past 30 days, for about how many days did poor physical or mental health keep you from doing your usual activities, such as self-care, work, school, or recreation?

The self-reported general health question (HSD010) has the response options "excellent," "very good," "good," "fair," "poor," "don't know," or "refused." For the other three variables that ask the participant for the number of unhealthy days, individuals can respond with any number between 0 and 30, as well as "don't know" and "refused" (CDC, 2013d).

Prior to using the response variables in analyses, distributions of the three healthy days variables and the general health variable responses were investigated in order to make decisions on whether to use them as continuous variables or as variables collapsed into categories. The CDC recommends dichotomizing the general health condition variable into good/excellent health and fair/poor health when researching HRQOL (CDC, 2012), although some researchers such as Cui, Zack, and Zahran (2015) group HRQOL into three or more categories. 
The next several sections describe the predictor variables chosen for this thesis and why these variables were selected for the regression models.

\subsubsection{Diet Quality Predictor Variables}

In order to acquire information regarding diet quality and intake, the dietary interview component of NHANES was used for this thesis. The purpose of the dietary interview in NHANES is to collect food and beverage intake from all participants to assess dietary behaviors and their possible relationship to health. In addition, obtaining detailed dietary information helps to gain insight on food patterns, diet quality, and total nutrient intake in the non-institutionalized US population. The first dietary interview was conducted in the MEC in-person, which consisted of collecting the amount of food and beverages consumed in the past 24-hours. Once the first interview was complete, a phone follow-up interview was scheduled 3-10 days after. All participants who completed the first 24-hour recall were eligible for the phone interview (CDC, 2009).

The dietary interview data files used for this research were Individual Foods and Total Nutrient Intakes for day one and day two. The Individual Foods Files contain detailed information about the foods and beverages consumed by the individual in the past 24 hours, which includes a description, the amount, and nutrient content for each food item. The Total Nutrient Files contain a summary of all nutrients consumed by each participant. In addition, information on any special diets the individual was on and if the amount of food reported was usual, less than usual, or more than the usual amount was also collected (CDC, 2014b). Only participants with two days of dietary intake were included in this research. For this thesis, we were not interested in a specific food or nutrient but rather in a measure of overall diet quality. The NHANES dietary interview component data can be used to calculate an overall measure of diet quality, the Healthy Eating Index $(\mathrm{HEI})$, as described in the next section. A self-perceived diet quality variable is in a different NHANES questionnaire, as described later. 


\subsubsection{Healthy Eating Index-2010 Overview, Background, and Uses}

The Healthy Eating Index-2010 (HEl-2010), developed by the United States Department of Agriculture Center for Nutrition Policy and Promotion (USDA-CNPP), is a tool that measures overall diet quality and can be calculated using the dietary data from participants in NHANES. The HEI-2010 was designed to reflect compliance with the 2010 Dietary Guidelines for Americans; guidelines are updated and released every 5 years (USDA-CNPP, 2014). The HEI-2010 has been validated and is a reliable indicator of diet quality (Guenther et al., 2014). Twelve components are added to create the total index score, which are total fruit, whole fruit, total vegetables, greens and beans, whole grains, dairy, total protein foods, seafood and plant proteins, fatty acids, refined grains, sodium, and empty calories. A higher score, which can reach a maximum of 100 points, is indicative of better diet quality and closer compliance with the 2010 Dietary Guidelines for Americans (USDA-CNPP, 2013). To calculate the twelve HEI-2010 component scores, the tool calls upon the FPED (Food Patterns Equivalents Database), which converts the food and beverage items recorded in the dietary interview section of NHANES into 37 Food Patterns (FP) components, for day one and day two of dietary data. The FP components are measured in cup equivalents for fruits, vegetables, and dairy, ounce equivalents for grains and proteins, teaspoon equivalents for added sugars, gram equivalents for solid fats and oils, and number of alcoholic drinks (United States Department of Agriculture Agricultural Research Service [USDA-ARS], 2014). A list of all 37 FP components is in Appendix G. These USDA food patterns are utilized to create the scoring standards for each of the $12 \mathrm{HEI}-2010$ components, which includes 9 adequacy and 3 moderation categories. The maximum scoring standards for cup, ounce, or teaspoon equivalents are per 1,000 calories, and any intake below that is a fraction thereof with a minimum score of zero (Guenther et al., 2013). The $12 \mathrm{HEI}-2010$ components and scoring standards for each are listed in Table 3.1. 
Table 3.1 Healthy Eating Index-2010 Components and Scoring Standards

\begin{tabular}{|c|c|c|c|}
\hline Component & Maximum Score & $\begin{array}{l}\text { Standard for } \\
\text { Maximum Score }\end{array}$ & $\begin{array}{l}\text { Standard for } \\
\text { Minimum Score of } \\
\text { Zero }\end{array}$ \\
\hline \multicolumn{4}{|l|}{$\begin{array}{l}\text { Adequacy } \\
\text { Components }\end{array}$} \\
\hline Total Fruit & 5 & $\begin{array}{l}\geq 0.8 \text { cup eq. per } \\
1,000 \text { kcal }\end{array}$ & No fruit \\
\hline Whole Fruit & 5 & $\begin{array}{l}\geq 0.4 \text { cup eq. per } \\
1,000 \text { kcal }\end{array}$ & No whole fruit \\
\hline Total Vegetables & 5 & $\begin{array}{l}\geq 1.1 \text { cup eq. per } \\
1,000 \text { kcal }\end{array}$ & No vegetables \\
\hline Greens and Peas & 5 & $\begin{array}{l}\geq 0.2 \text { cup eq. per } \\
1,000 \text { kcal }\end{array}$ & $\begin{array}{l}\text { No dark greens or } \\
\text { peas }\end{array}$ \\
\hline Whole Grains & 10 & $\begin{array}{l}\geq 1.5 \text { oz. eq. per } 1,000 \\
\text { kcal }\end{array}$ & No whole grains \\
\hline Dairy & 10 & $\begin{array}{l}\geq 1.3 \text { cup eq. per } \\
1,000 \text { kcal }\end{array}$ & No dairy \\
\hline Total Protein Foods & 5 & $\begin{array}{l}\geq 2.5 \text { oz. eq. per } 1,000 \\
\text { kcal }\end{array}$ & No protein foods \\
\hline $\begin{array}{l}\text { Seafood and Plant } \\
\text { Proteins }\end{array}$ & 5 & $\begin{array}{l}\geq 0.8 \text { oz. eq. per } 1,000 \\
\text { kcal }\end{array}$ & $\begin{array}{l}\text { No seafood or plant } \\
\text { proteins }\end{array}$ \\
\hline Fatty Acids & 10 & $\begin{array}{l}\text { (PUFAs }+ \\
\text { MUFAs)/SFAs } \geq 2.5^{\mathrm{a}}\end{array}$ & $\begin{array}{l}(\text { PUFAs }+ \\
\text { MUFAs)/SFAs } \leq 1.2\end{array}$ \\
\hline \multicolumn{4}{|l|}{$\begin{array}{l}\text { Moderation } \\
\text { Components }\end{array}$} \\
\hline Refined Grains & 10 & $\begin{array}{l}\leq 1.8 \text { oz. eq. per } 1,000 \\
\text { kcal }\end{array}$ & $\begin{array}{l}\geq 4.3 \text { oz. eq. per } 1,000 \\
\text { kcal }\end{array}$ \\
\hline Sodium & 10 & $\begin{array}{l}\leq 1.1 \text { gram per } 1,000 \\
\text { kcal }\end{array}$ & $\begin{array}{l}\geq 2.0 \text { grams per } 1,000 \\
\text { kcal }\end{array}$ \\
\hline Empty Calories & 20 & $\leq 19 \%$ of energy & $\geq 50 \%$ of energy \\
\hline
\end{tabular}

From Guenther et al., 2013.

${ }^{a}$ PUFAs=polyunsaturated fatty acids, MUFAs=monounsaturated fatty acids, SFAs=saturated fatty acids

The HEI-2010 can be used at various levels for research on adherence to dietary guidelines, including the national food supply, food processing, community food 
environment, and individual food intake (National Cancer Institute [NCl], 2015). With NHANES data, an HEI can be calculated for each individual from each 24-hour recall, or a 2-day average can be created. However, there are concerns about how reflective two days of dietary data are of long-term usual intake in an individual. At an individual level, there are few options to minimize the effects of measurement error for NHANES dietary intake data due to the limitation of having only two days of data available. Therefore, the $\mathrm{NCl}$ developed a "population ratio method" to estimate mean HEl scores for a population, subpopulation, or group based on one day of dietary recall data. This method is intended to minimize the limitations of 24-hour dietary recall data by creating mean HEl scores that are representative of a population's usual intake ( $\mathrm{NCl}, 2015)$. However, data at an individual level were of interest for this thesis in order to use $\mathrm{HEI}$ as a predictor variable in regression models.

Because of this interest, preliminary work was done to rationalize the use of individual data for the two days of dietary intake data available. For this research in particular, the HEI-2010 total and component scores were calculated at the individual level to determine diet quality in older adults who participated in the NHANES survey from 2009-2010 and 2011-2012; the $\mathrm{NCl}$ population ratio method was used as well. The various methods used to calculate $\mathrm{HEI}$ scores as well as the justification for each method are described below.

For this thesis research, HEI was looked at both at a subpopulation and at an individual level. For the former, the National Cancer Institute $(\mathrm{NCl})$ describes a step-bystep process using SAS code to estimate mean HEI scores for a population, subpopulation, or group (NCI, 2015). The HEI-2010 SAS code for NHANES data files provided by the $\mathrm{NCl}$ for the population ratio method was used as a guide, and modified for this thesis research. Modifications to the code were required to account for the specific years of data, sample population, subsamples, and to calculate HEls for each 
individual rather than group means only. This SAS program is divided into four sections that are described in detail below.

The first section involved calculating at the individual level using NHANES participants' dietary data to obtain the variables needed to calculate the HEI-2010 scores. The FPED SAS datasets for 2009-2010 and 2011-2012 were read into the SAS program, which contains the individual food consumed, dietary sample weights, and the $12 \mathrm{HEI}$ components. Next, the total nutrient files for day one and day two in NHANES were read in, which contains the total calories, carbohydrates, fat, and sodium intake for all participants. The demographics data files from NHANES were then read into the SAS program, which includes the participant identification number, age, gender, marital status, masked variance pseudo-primary sampling units (PSUs), and masked variance pseudo-stratum to account for the complex survey design. The FPED individual foods, total nutrient, and demographics datasets were combined into one dataset, and the age variable was specified to only include participants 60 and older in order to analyze the older adult participants. Seven additional variables were calculated to coincide with the HEI categories: MONOPOLY, ALLMEAT, SEAPLANT, ADDSUGC, SOLFATC, MAXALCGR, and EMPTYCAL10. MONOPOLY was monounsaturated and polyunsaturated fat intake added together. ALLMEAT was all the types of meat in the FPED dataset combined, which included cured meat, organ meat, poultry, eggs, nuts and seeds, and soy. SEAPLANT included seafood high in omega-3 fatty acids, seafood low in omega-3 fatty acids, soy, and nuts and seeds. ADDSUGC was foods defined as added sugars in teaspoon equivalents multiplied by $16 \mathrm{kcal} / \mathrm{gram}$ to obtain calories from added sugars. SOLFATC was solid fats in grams multiplied by $9 \mathrm{kcal} / \mathrm{gram}$ to obtain the calories from solid fat. MAXALCGR was the maximum grams of alcohol based on calorie intake, which was equal to $13^{*}$ (calories/1000). If the participants consumed less than the maximum, then their extra alcohol in grams intake equaled zero. If their alcohol intake 
was greater than the maximum, then the extra calories from alcohol were obtained by multiplying $7 \mathrm{kcal} / \mathrm{gram}$ by the difference of total alcohol intake and the maximum grams of alcohol based on calorie intake. To calculate the number of empty calories consumed for the variable EMPTYCAL10, ADDSUGC, SOLFATC, AND MAXALCGR were combined for the HEl empty calorie component (NCI, 2015).

Section two of the $\mathrm{NCl}$ population ratio method was the calculation of weighted means, a variance-covariance matrix, and generation of a Monte Carlo dataset. The purpose of generating a Monte Carlo dataset is to estimate mean usual intakes in a population or subpopulation from 24 -hour recalls. To do this, 10,000 sample people are simulated from each NHANES participant to allow standard errors to be estimated, taking the complex survey design of NHANES into account. The generated Monte Carlo dataset was used for the next section ( $\mathrm{NCl}, 2015)$.

The third section in calculating mean HEl scores was the allocation of beans and peas (legumes) and application of the HEI-2010 scoring algorithm using SAS macros ( $\mathrm{NCl}, 2015)$. Macros consist of pre-written code that are read into the program by using the "\%INCLUDE" statement and the pathway to the file that the macros are saved under (SAS Institute, 2015). The first macro read in was the Legume Allocation Macro, Version 1.1, which contained code that allocated legumes (beans and peas) into either the Total Vegetables category or the Total Proteins (seafood and plant) category. If the individual did not meet the standard for total proteins of $2.5 \mathrm{oz}$ equivalents per 1000 calories, the legumes counted towards the total protein category. If the daily protein recommendation was met based on the individual's caloric intake, the legumes were allocated to the total vegetables category. Once the beans and peas were allocated to the appropriate category using the Monte Carlo dataset the HEI-2010 Scoring Macro was applied, which calculated densities for each HEI-2010 component and applied the scoring algorithm afterwards (NCl, 2015). 
The fourth and final section was the calculation of HEI-2010 component scores, total scores, standard errors, and confidence intervals. The univariate procedure was used to calculate confidence intervals and percentiles. The means procedure was then used to calculate the minimum, maximum, mean, standard error, and standard deviation of the $12 \mathrm{HEl}$ component and total scores. The datasets created from these two procedures were then merged together to prepare and display the results $(\mathrm{NCl}, 2015)$. The SAS code that accompanies this 4-step procedure, as modified for this research, is in Appendix $\mathrm{H}$.

The HEI procedure described above is the method for estimating subpopulation mean HEI total scores, which is defined as NHANES participants aged 60 and above for this thesis. But in order to include $\mathrm{HEI}$ as a predictor variable for this thesis, HEI scores were calculated for each person, because individual HEI scores are needed in order to examine relationships between HEI scores and other diet and HRQOL variables. To calculate HEI scores per individual, the FPED SAS datasets for 2009-2010 and 20112012 with the individual foods were read into SAS. The total nutrient and demographics datasets were also read in and merged with the FPED individual foods dataset. The same variables (MONOPOLY, ALLMEAT, SEAPLANT, ADDSUGC, SOLFATC, MAXALCGR, and EMPTYCAL10) created in the population ratio method were also created for this individual method. The SAS code from the macros was taken and integrated into the program to allocate legumes to the Total Protein or Total Vegetables HEI categories. The total HEI score was obtained by adding up all the component scores per individual, and the SAS SURVEYMEANS procedure was used to calculate descriptive statistics. The SAS code used to carry out this procedure for individual HEI scores is in Appendix I.

As mentioned, there are concerns with using only one or two days of dietary data when usual intakes are of interest. Therefore, the researchers at the $\mathrm{NCl}$ suggest to 
interpret results with caution, as there is potential for bias when interpreting results $(\mathrm{NCl}$, 2015). For this thesis, several approaches were taken to investigate whether the individual HEI scores (or a 2-day average of individual scores) would be reasonable to use. First, day one and day two $\mathrm{HEI}$ scores were calculated using the $\mathrm{NCl}$ population ratio method to determine if the mean scores were similar to one another. Since NHANES day one dietary data is conducted in the Mobile Examination Center (MEC) and day two data is collected through a phone interview 3-10 days later (CDC, 2014a), similar average population scores between the two days would help justify using both days of data because it would show similarity of data gathered by the two methods.

As previously mentioned, these usual intake HEI mean scores for day one and day two cannot be used in a regression model as predictors because individual HEI scores are needed, which was the original interest in the HEI variable. Therefore, further analyses of HEI scores for each NHANES participant based on two 24-hour recalls were conducted in order to justify use of HEI-2010 scores as a diet-related predictor variable.

The first approach was to examine the correlation between day one and day two of an individual's dietary data. To do this, a correlation coefficient with a scatter plot was calculated to determine the strength of the association between day one and day two total HEI scores. If there was an association between the two, that would validate using only two days worth of dietary data because it would suggest that there is some consistency of diet quality (akin to usual intake). In addition, quintiles of HEI for day one vs. day two were calculated to visualize whether or not an individual was in the same quintile for both days. If diet is relatively consistent day-to-day, participants who were in the first quintile or at least the first few quintiles of $\mathrm{HEI}$ on day one should be in the first quintiles on day two if the HEI score represents "usual" intake, which would be another indicator of consistency between the two 24-hour recalls. Likewise, if day one and day two intakes were consistent, few individuals in quintile one of day one would be in 
quintile five of day two, and few in quintile five of day one would be in quintile one of day two.

Once comparisons of day one vs. day two individual scores were conducted, various methods were considered in order to obtain average HEI total scores per individual. The first method to obtain individual HEI scores is to get a 2-day average of individual HEl scores when two 24-hour recalls are available for each participant. To do this, each $\mathrm{HEl}$ component score from day one and day two of individual dietary data were added together and divided by 2 . Then the average of each of the two-day component scores were added together to get the 2-day average total HEI score. A second method for obtaining average HEI total scores for individuals is by taking the average over two days. To do this, each food intake variable for day one and day two were added together before the legume allocation and HEI scoring algorithm were applied for each component. Once each component was calculated, they were added together to obtain the total HEI score over two days. Average HEI scores obtained by the two methods were examined and compared with analyses conducted by Freedman and colleagues (2010) to determine which calculation was most appropriate for the logistic regression models in this thesis.

\subsubsection{Self-Perceived Diet Quality}

The Diet Behavior and Nutrition Questionnaire includes data on nutrition-related topics, ranging from food allergies to meal program utilization (CDC, 2015b). Because this thesis was looking at the relationship between dietary quality and health-related quality of life, the variable "how healthy is the diet" was of interest. The question associated with this variable is as follows:

DBQ700: In general, how healthy is your overall diet?

Participants could choose from the responses "excellent," "very good," "good," "fair," "poor," "refused," or "don't know" to answer this question. This variable was 
chosen to analyze self-reported diet quality among NHANES participants and its potential association with HRQOL. In addition, the association between this selfperceived diet quality variable and actual diet quality was looked at to determine if there was any correlation between the two. A strong correlation would be further justification that $\mathrm{HEI}$ calculated from the 24 -hour recalls was a valid estimator of usual diet quality. The following section describes other nutrition-related predictor variables that were chosen for the main statistical analyses for this thesis research.

\subsubsection{Other Diet-Related Predictor Variables}

One of the main objectives of NHANES is to assess the nutritional status of adults and children in the US (CDC, 2014a), which is why there are multiple questionnaires with variables related to diet and nutrition. As older adults age, physical and functional changes begin to occur, which can impair their ability to eat and enjoy a meal and can affect their quality of life as a result (Bernstein \& Munoz, 2012). For these reasons, the diet-related variables available in NHANES and chosen as predictors for this research were meal program use, difficulties with food-related activities, and poor appetite and overeating.

The Diet Behavior and Nutrition Questionnaire contains questions related to meal program use in addition to self-perceived diet quality. Because certain meal programs are specific to the older adult population, the following two variables were included:

DBQ301: In the past 12 months, did you receive any meals delivered to your home from community programs "Meals on Wheels," or any other programs? DBQ330: In the past 12 months, did you go to a community program or senior center to eat prepared meals?

These questions are specifically for adults aged 60 and above, and the response options are "yes," "no," "don’t know," and "refused” (CDC, 2015b). 
The Physical Functioning Questionnaire assesses physical, mental, and emotional difficulties caused by functional limitations (CDC, 2015c). It's important to assess and analyze seniors' meal-related functioning because functional impairments can have an effect on daily living and HRQOL (Bernstein \& Munoz, 2012). Since this thesis focuses on dietary factors in relation to quality of life, these nutrition-related physical functioning variables were chosen:

PFQ061G: By yourself and without using any special equipment, how much difficulty do you have preparing your own meals?

PFQ061K: By yourself and without using any special equipment, how much difficulty do you have eating, like holding a fork, cutting food, or drinking from a glass?

The response options for both questions are "no difficulty," "some difficulty," "much difficulty," "unable to do," "do not do this activity," "refused," and "don't know." (CDC, 2015d).

The mental health-depression screener questionnaire, which is part of the MEC interview, measures depression using the Patient Health Questionnaire (PHQ). The $\mathrm{PHQ}$ is a 9-item screening tool with questions regarding depressive symptoms occurrences in the past two weeks. Since dietary and diet-related factors are the main independent variables for this research, the following variable was chosen as part of the regression model:

DPQ050: Over the last two weeks, how often have you been bothered by the following problems: poor appetite or overeating?

Participants either responded with "not at all," "several days," "more than half the days." "nearly every day," "refused," or "don't know" (CDC, 2015c). For all diet-related predictor variables, initial analyses were conducted to look at the number of older adults in each response category, as well as weighted percentages. These analyses helped determine 
the distribution of each variable and if modifications needed to be made before including them in the regression models.

\subsubsection{Other Predictor Variables}

The other predictor variables that were relevant for this research are from the NHANES demographics data file. Most of these variables are characteristics of the participants that need to be taken into account before analyzing the relationship between diet and health-related quality of life, because they can be associated with both the independent and dependent variables. In addition, they are standard covariates used in studies of predictors of HRQOL in older adults (Ford et al., 2014; Frazier-Wood et al., 2015; Nguyen et al., 2015). These variables addressed were gender, race, education, marital status, total number of people in the household, and income. Table 3.2 lists the possible responses for each of these predictor variables.

\subsection{Research Questions and Statistical Analyses}

This next section describes the descriptive analyses that were conducted for the diet quality variables as well as the research questions and regression models that were used to answer those research questions.

\subsubsection{Population Characteristics}

In addition to participant characteristics, the demographics data files in the NHANES survey cycles 2009-2010 and 2011-2012 also contain the sample weights, masked variance pseudo-primary sampling units (PSUs), and masked variance pseudostratum that must be used in all data analyses to account for the complex survey design.

Each survey cycle has two sample weights, one for the household interview (WTINT2YR) and one for the MEC exam (WTMEC2YR) (CDC, 2013e). In order to attain proper variance estimation, the weight of the smallest subsample must be used, which in this case was any variables conducted in the MEC. Therefore, the sample weight WTMEC2YR was used for this particular data analysis as opposed to the sample weight 
WTINT2YR (CDC, 2013c). To obtain a 4-year sample weight, the variable MEC4YR was created by multiplying 0.5 times the WTMEC2YR for each 2-year survey cycle (CDC, 2013f).

Table 3.2 Possible Responses for Demographic Predictor Variables, NHANES 20092012

\begin{tabular}{|c|c|c|}
\hline Variable & Label & Responses \\
\hline RIAGENDR & Gender & $\begin{array}{l}\text { Male (referent) } \\
\text { Female }\end{array}$ \\
\hline RIDRETH1 & Race/Hispanic Origin & $\begin{array}{l}\text { Mexican American } \\
\text { Other Hispanic } \\
\text { Non-Hispanic White (referent) } \\
\text { Non-Hispanic Black } \\
\text { Other Race-including Multiracial }\end{array}$ \\
\hline DMDEDUC2 & Education Level-Adults 20+ & $\begin{array}{l}\text { Less than 9th Grade (referent) } \\
\text { High School Grad/GED or equivalent } \\
\text { Some College or AA degree } \\
\text { College Graduate or above }\end{array}$ \\
\hline DMDMARTL & Marital Status & $\begin{array}{l}\text { Married (referent) } \\
\text { Widowed } \\
\text { Divorced } \\
\text { Separated } \\
\text { Never Married } \\
\text { Living with partner }\end{array}$ \\
\hline DMDHHSIZ & Total Number of People in the Household & $\begin{array}{l}\text { 1-6 Range of Values } \\
7 \text { or more }\end{array}$ \\
\hline INDFMPIR & Ratio of Family Income to Poverty & $\begin{array}{l}0 \text { to } 4.99 \text { Range of Values } \\
\text { Value greater than or equal to } 5\end{array}$ \\
\hline
\end{tabular}

Data from NHANES Demographics Data Files

For data involving the NHANES dietary interview component, the dietary day one sample weight (WTDRD1) or two-day sample weight (WTDR2D) was used (CDC, 2014b), depending on the number of days analyzed. Again, to obtain a 4-year sample weight, WTDRD1 and WTDR2D from each 2-year cycle were multiplied by 0.5 (CDC, 2013f). Initial analyses on relevant demographic variables were conducted in the same way as the diet-related predictor variables to determine counts of older adults, weighted percentages, and the distribution of responses. In addition, population characteristics 
were calculated and presented by HRQOL response category (excellent, very good, good, fair, and poor).

\subsubsection{Diet Quality Descriptive Analyses}

To examine actual diet quality within health-related quality of life categories, descriptive analyses were conducted using the $\mathrm{NCl}$ population ratio method as previously described. Actual diet quality, measured by mean HEI scores, were calculated for each response category to the general health question variable (HSD010) in the Current Health Status Questionnaire in NHANES, with the HRQOL category specified as the subpopulation of interest using the $\mathrm{NCl}$ method. Day one and day two mean HEl scores were calculated separately for the "excellent," "very good," "good," "fair," and "poor" HRQOL responses (CDC, 2013d) to determine potential HEI score differences between the days and the general health condition responses. In addition to these 5 responses, participants could also respond with "don't know" and "refused." Because these options are considered unavailable for analysis, they were recoded as missing values before analyzing the results (CDC, 2013g).

Self-perceived diet quality and actual diet quality were examined in a similar way. The self-perceived diet quality variable (DBQ700) in the Diet Behavior and Nutrition Questionnaire in NHANES had the responses "excellent," "very good," "good," "fair," and "poor" (CDC, 2015b), and mean HEI scores using the $\mathrm{NCl}$ population ratio method were calculated for each response category for day one of day two of dietary data, with expectations that population mean HEl scores would be lowest in the fair and poor response categories. Individual mean HEI scores were also calculated by response to the self-perceived diet quality question using the HEl averages over two days, the 2-day average, and day one and two averages separately. This way, the proportion of older adults aged $60+$ in each self-perceived diet quality category can be seen since each score was calculated per individual, in order to examine differences in frequency 
between each category. Because self-perceived diet quality should reflect usual diet quality, association of individual HEI with self-perceived quality would provide further justification for including $\mathrm{HEl}$ as a predictor variable.

\subsubsection{Regression Models}

This section lists the main research question for this thesis and the regression models associated with it. In addition, the hypotheses for each model and the main predictor variables, covariates, and outcome variables are described below.

The broad research question was as follows: "Is diet quality associated with quality of life measures in older adults aged 60+?" Regression models were constructed with 4 quality of life outcome measures: overall HRQOL, number of physically unhealthy days, number of mentally unhealthy days, and number of inactive days due to physical or mental health issues.

Overall HRQOL was the main outcome measure for this thesis research because it was a general health question asked to NHANES participants, while the healthy days measures only asked about the number of unhealthy days in the past 30 days. A global HRQOL variable gave more information regarding how participants felt about their overall health in general, rather than solely focusing on a recent number of days that could be vary depending on when the data were collected.

Specifically, the main research question was as follows: Is diet quality associated with HRQOL, after controlling for the key covariates? The hypothesis is that diet quality, both actual and self-perceived, is associated with HRQOL, and a higher diet quality is associated with higher (better) HRQOL, after adjusting for relevant covariates. There were 3 models with the global HRQOL outcome measure, which had the binary outcome of good or better HRQOL vs. fair or poor HRQOL. Model 1, called the Continuous HEI Model, included the average total $\mathrm{HEl}$ individual scores over two days as a continuous variable along with all the nutrition-related covariates and other demographic variables 
described previously. Model 2, called the Categorical HEI Model, had the same covariates and other variables as Model 1, but the average total HEl individual scores over two days variable was made categorical by separating the HEI scores into quartiles. Model 3, called the Self-Perceived Diet Quality Model, included the same covariates as Models 1 and 2 along with the "how healthy is the diet" variable, which was dichotomized into good diet quality (good, very good, or excellent) vs. worse (fair or poor) diet quality. Logistic regression was used by running PROC SURVEYLOGISTIC in SAS to get odds ratios, point estimates, $95 \%$ confidence intervals, and $p$-values for the main predictors and covariates for the binary HRQOL outcome. The covariates included in each model were meals delivered, meals eaten at a senior/community center, poor appetite/overeating, and meal prep difficulty, age, gender, race, education, marital status, income, and living alone.

Interactions between the main predictor variable (the diet quality variable of average individual $\mathrm{HEI}$ total scores over two days) and each of the covariates in Model 1, the Continuous HEI Model, were investigated to determine possible effects on the interpretation and outcome of the model. To do this, every interaction term was included in the model the first time it ran in SAS, and the interaction with the highest p-value was removed. The model was run through again, and the next interaction with the highest $p-$ value was removed. This backward elimination process continued until only statistically significant $(p<0.05)$ interactions were left in the model (Hardy \& Bryman, 2009).

For the 3 healthy days outcome measures, the research question was, "Controlling for key covariates, is HEl associated with healthy days?" The hypothesis is that $\mathrm{HEI}$ is associated with number of physically, mentally, and inactive days due to physical/mental issues, specifically that a high quality diet (high HEI score) is associated with a lower amount of unhealthy days. The Physically Unhealthy Days Model included the average total $\mathrm{HEI}$ individual scores over two days as a continuous variable, along 
with all the nutrition-related covariates and other demographic variables described previously. The outcome variable included the number of physically unhealthy days, which was dichotomized into 0-15 days (healthier days) and 16-30 days (unhealthier days). The Mentally Unhealthy Days Model included the continuous HEI variable, all key covariates and the binary mentally unhealthy days outcome (0-15 days vs. 16-30 days). The Inactive Days due to Physical/Mental Health Issues Model included HEI, covariates, and the number of inactive days outcome (0-15 days vs. 16-30 days).

Some of the nutrition-related covariates were collapsed before including them in the logistic regression models. The meal preparation difficulty variable, which had the responses "no difficulty," "some difficulty," "much difficulty," "unable to do," and "do not do this activity," were collapsed into three categories" "difficulty," "no difficulty," and "do not do this activity" because of the low number of participants in the "some difficulty" and "much difficulty" response categories. For the government meal utilization variables, which included meals delivered and meals eaten at a Senior/community center, the response options were either "yes" or "no," and this variable was kept as a categorical variable in this form. The poor appetite/overeating variable in the NHANES mental health-depression screener questionnaire had the response options "not at all," "several days," "more than half the days," and "nearly every day" when asked if participants had been bothered by poor appetite or overeating in the past two weeks. These responses were kept as four categories in the regression model.

The covariates, as previously mentioned, that were included in the regression models were age, gender, race, education, marital status, income, and living alone. Since age was looked at categorically throughout this thesis, age was categorized as 6069 year olds, $70-79$ year olds, and 80+ year olds for the logistic regression models. Age could not be used as a continuous variable because NHANES participants aged 80 and above are top coded as 80 (CDC, 2013c). Gender, race, and education responses were 
all kept in the regression models, as described in Table 3.2. Marital status response categories were collapsed into "married or living with partner" and "not married or living with partner." Married included the responses "living with partner" and "married," and not married included the responses "widowed," "divorced," and "separated." Income was measured using the ratio of family income to poverty variable, which was calculated by dividing family or individual income by the poverty guidelines for that year. The poverty guidelines vary by family size and geographic location (CDC, 2013e). As listed in Table 3.2, response options for this variable included a range of values from 0 to 4.99 and any ratio equal to 5 or above was top coded as 5 to retain anonymity. Since the Commodity Supplemental Food Program (CSFP), a nutrition program for low-income adults, has eligibility requirements at or below $130 \%$ of the Poverty Guidelines (CSFP, 2014), the ratio of family income to poverty was collapsed into poverty (1.3 and below) and no poverty (1.4 and above.) The living alone variable was based off the total number of people in the household, which had the response options 1-6 and 7 or more. Participants who responded with " 1 " as the number of people in the household were categorized as living alone, and every other response was categorized as not living alone. 


\section{Chapter 4: Results}

All statistical analyses were conducted using SAS version 9.4 (SAS Institute, 2015). The results from the analyses described in Chapter 3 for the HRQOL, diet quality, diet-related, and other relevant predictor variables selected for this thesis are reported in this chapter.

\subsection{Population Descriptive Data}

Demographic characteristics of older adults aged 60+ in the NHANES survey cycles 2009-2010 and 2011-2012 are displayed in Table 4.1, which shows the number of older adults in each demographic variable response category by age group $(60-69,70$ 79 , and $80+$ ) and the weighted percentages of older adults in each category. Weighted frequencies were calculated in order to look at potential differences and patterns in demographic responses as older adults age. Overall, $78 \%$ of older adults were nonHispanic White, $29 \%$ had an AA degree or completed some college, and $63 \%$ were married. More than $55 \%$ of all subjects $60+$ were female, and $23 \%$ lived alone.

Table 4.2 shows the number and weighted percentages of older adults in each HRQOL category (excellent, very good, good, fair, and poor). When the NHANES 20092010 and 2011-2012 data cycles were combined for this thesis, there were 3,692 older adults aged $60+$ included for results and data analysis. However, the general health condition variable included 3,392 older adult participants total, with 300 older adults with "missing" responses. Regardless, the calculated sample weights take missing responses into account and adjust accordingly. Overall, older adults most often stated that their general health condition was "good" followed by very good, fair, excellent, and poor. For the race/ethnicity demographic variable, Non-Hispanic Whites had the highest percentage of "excellent" (12.9\%) and "very good" responses (34.9\%). Additionally, $53.5 \%$ of Mexican Americans and $33.4 \%$ of non-Hispanic Blacks rated their overall 
health as "fair" or "poor," while only $15.6 \%$ of non-Hispanic Whites rated their overall health as "fair" or "poor."

Table 4.1 Demographic Information of Older Adults 60+ by Age Group in NHANES Data Cycles 2009-2010 and 2011-2012

\begin{tabular}{|c|c|c|c|c|c|}
\hline Characteristic & & $60-69$ years & 70-79 years & $80+$ years & $\begin{array}{c}\text { All Subjects } \\
60+\end{array}$ \\
\hline \multicolumn{6}{|l|}{ Gender } \\
\hline Male & $\%\left(n^{a}\right)$ & $47.6(913)$ & $44.4(565)$ & $37.2(342)$ & $44.7(1820)$ \\
\hline Female & $\%(n)$ & $52.4(902)$ & $55.6(590)$ & $62.8(380)$ & $55.3(1872)$ \\
\hline \multicolumn{6}{|l|}{ Race } \\
\hline $\begin{array}{l}\text { Mexican } \\
\text { American }\end{array}$ & $\%(n)$ & $4.2(282)$ & $3.6(94)$ & $2.4(36)$ & $3.7(412)$ \\
\hline $\begin{array}{l}\text { Other } \\
\text { Hispanic }\end{array}$ & $\%(n)$ & $3.6(245)$ & $3.9(90)$ & $2.8(35)$ & $3.5(370)$ \\
\hline $\begin{array}{l}\text { Non- } \\
\text { Hispanic } \\
\text { White }\end{array}$ & $\%(n)$ & $76.6(644)$ & $77.5(641)$ & $85.1(529)$ & $78.3(1814)$ \\
\hline $\begin{array}{l}\text { Non- } \\
\text { Hispanic } \\
\text { Black }\end{array}$ & $\%(n)$ & $9.5(488)$ & $9.9(242)$ & $5.4(78)$ & $8.9(808)$ \\
\hline $\begin{array}{l}\text { Other Race/ } \\
\text { Multiracial }\end{array}$ & $\%(n)$ & $6.1(156)$ & $5.1(88)$ & $4.4(44)$ & $5.5(288)$ \\
\hline \multicolumn{6}{|l|}{ Education } \\
\hline $\begin{array}{l}\text { Less than } 9^{\text {th }} \\
\text { grade }\end{array}$ & $\%(n)$ & $6.5(285)$ & $10.6(198)$ & $15.7(145)$ & $9.3(628)$ \\
\hline $9-11^{\text {th }}$ grade & $\%(n)$ & $9.1(259)$ & $15.8(214)$ & $15.2(115)$ & $12.1(588)$ \\
\hline $\begin{array}{l}\text { High school } \\
\text { graduate/GE } \\
\text { D or } \\
\text { equivalent }\end{array}$ & $\%(n)$ & $20.8(387)$ & $25.7(264)$ & $24.8(165)$ & $22.9(816)$ \\
\hline $\begin{array}{l}\text { Some } \\
\text { college or } \\
\text { AA Degree }\end{array}$ & $\%(n)$ & $31.6(503)$ & $25.5(263)$ & $26.7(170)$ & $29.0(936)$ \\
\hline $\begin{array}{l}\text { College } \\
\text { graduate or } \\
\text { Above }\end{array}$ & $\%(n)$ & $31.9(378)$ & $22.5(214)$ & $17.5(122)$ & $26.7(714)$ \\
\hline
\end{tabular}


Table 4.1, continued

\begin{tabular}{llccc}
\hline Characteristic & $60-69$ years & $70-79$ years & $80+$ years & $\begin{array}{c}\text { All Subjects } \\
60+\end{array}$ \\
\hline
\end{tabular}

\section{Marital Status}

\begin{tabular}{|c|c|c|c|c|c|}
\hline Married & $\%(n)$ & $66.9(1034)$ & $64.4(669)$ & $45.4(313)$ & $62.5(2016)$ \\
\hline Widowed & $\%(n)$ & $9.1(218)$ & $21.0(281)$ & 46.7 (347) & $19.1(846)$ \\
\hline Divorced & $\%(n)$ & $14.8(316)$ & $8.8(121)$ & $4.2(31)$ & $11.2(468)$ \\
\hline Separated & $\%(n)$ & $1.4(58)$ & $1.0(23)$ & $0.7(7)$ & $1.1(88)$ \\
\hline $\begin{array}{l}\text { Never } \\
\text { Married }\end{array}$ & $\%(n)$ & $4.5(121)$ & $3.3(44)$ & $2.2(19)$ & 3.8 (184) \\
\hline $\begin{array}{l}\text { Living with } \\
\text { Partner }\end{array}$ & $\%(n)$ & $3.3(66)$ & $1.5(16)$ & $0.8(4)$ & $2.3(86)$ \\
\hline iving Alone & & & & & \\
\hline Yes & $\%(n)$ & $19.6(379)$ & $21.8(282)$ & $36.6(262)$ & $23.1(923)$ \\
\hline No & $\%(n)$ & $80.4(1436)$ & $78.2(873)$ & $63.4(460)$ & 76.9 (2769) \\
\hline $\begin{array}{l}\text { atio of family } \\
\text { come to } \\
\text { overty }\end{array}$ & $\begin{array}{c}\text { Mean }(S E) \\
n\end{array}$ & $\begin{array}{c}3.4(0.09) \\
1636\end{array}$ & $\begin{array}{c}2.9(0.07) \\
1026\end{array}$ & $\begin{array}{c}2.5(0.09) \\
640\end{array}$ & $\begin{array}{c}3.1(0.07) \\
3302\end{array}$ \\
\hline
\end{tabular}

Data from NHANES 2009-2012 Demographics and Questionnaire Data Files

${ }^{a}$ All $n$ are analytic sample $n$; all $\%$ and mean data are weighted in order to represent the U.S. population. Weighting accounts for the probability of being selected for the sample, survey nonresponse, and differences between the sample and total U.S. population

Over $61 \%$ of those who were in poverty $(<130 \%$ of the Federal Poverty Level) reported their overall health as "excellent," "very good," or "good," while $84.8 \%$ of those who were not in poverty reported their overall health as "excellent," "very good," or "good." For the education demographic variable, older adult participants with a college degree or above had the highest percentage of "excellent" responses (21.7\%) and "very good" responses $(41.7 \%)$, and the lowest amount of "poor" responses $(1.4 \%)$. On the other hand, participants with less than $9^{\text {th }}$ grade education had the highest percentage of fair $(37.3 \%)$ and poor $(14.2 \%)$ responses. The percentages of older adults in each HRQOL response category were very similar between males and females. For the marital status 
demographic variable, the participants that were divorced had the highest percentage of "excellent" health (17.0\%), while "married" had the highest percentage of "very good" health (34.3\%) and "living with partner" had the lowest amount of "poor" health (3.3\%).

Table 4.2 Weighted Percentages of Health-Related Quality of Life Response Categories by Demographic Characteristics of Older Adults 60+ in NHANES Data Cycles 2009-2010 and $2011-2012$

\begin{tabular}{|c|c|c|c|c|c|}
\hline Characteristic & Excellent & $\begin{array}{l}\text { Very } \\
\text { Good }\end{array}$ & Good & Fair & Poor \\
\hline $\begin{array}{l}\text { All Subjects } \\
\qquad\left(n=3392^{a}\right)\end{array}$ & 11.3 & 30.8 & 37.9 & 16.2 & 3.9 \\
\hline $\begin{array}{l}\text { 60-69 years } \\
(n=1697)\end{array}$ & 13.0 & 32.9 & 36.3 & 14.1 & 3.8 \\
\hline $\begin{array}{l}70-79 \text { years } \\
(n=1133)\end{array}$ & 10.3 & 28.9 & 39.5 & 17.4 & 3.8 \\
\hline $\begin{array}{l}80+\text { years } \\
(n=642)\end{array}$ & 8.1 & 27.4 & 40.0 & 20.3 & 4.2 \\
\hline \multicolumn{6}{|l|}{ Gender } \\
\hline $\begin{array}{l}\text { Male } \\
(n=1679)\end{array}$ & 12.0 & 30.1 & 38.5 & 15.9 & 3.6 \\
\hline $\begin{array}{l}\text { Female } \\
(n=1713)\end{array}$ & 10.8 & 31.3 & 37.4 & 16.4 & 4.1 \\
\hline \multicolumn{6}{|l|}{ Race } \\
\hline $\begin{array}{l}\text { Mexican American } \\
(\mathrm{n}=371)\end{array}$ & 4.4 & 8.7 & 33.4 & 41.3 & 12.2 \\
\hline $\begin{array}{l}\text { Other Hispanic } \\
(n=346)\end{array}$ & 8.6 & 10.4 & 39.0 & 36.0 & 6.1 \\
\hline $\begin{array}{l}\text { Non-Hispanic } \\
\text { White } \\
(n=1688)\end{array}$ & 12.9 & 34.9 & 36.5 & 12.6 & 3.0 \\
\hline $\begin{array}{l}\text { Non-Hispanic } \\
\text { Black } \\
(n=737)\end{array}$ & 5.2 & 17.7 & 43.6 & 27.0 & 6.4 \\
\hline $\begin{array}{l}\text { Other Race/ } \\
\text { Multiracial } \\
(n=247)\end{array}$ & 3.5 & 18.3 & 52.8 & 20.7 & 4.6 \\
\hline
\end{tabular}


Table 4.2, continued

\begin{tabular}{|c|c|c|c|c|c|}
\hline Characteristic & Excellent & $\begin{array}{l}\text { Very } \\
\text { Good }\end{array}$ & Good & Fair & Poor \\
\hline \multicolumn{6}{|l|}{ Education } \\
\hline $\begin{array}{l}\text { Less than } 9^{\text {th }} \text { grade } \\
(n=553)\end{array}$ & 3.9 & 11.0 & 33.6 & 37.3 & 14.2 \\
\hline $\begin{array}{l}9-11^{\text {th }} \text { grade } \\
(n=529\end{array}$ & 3.4 & 19.5 & 45.6 & 25.8 & 5.6 \\
\hline $\begin{array}{l}\text { High school } \\
\text { graduate/ GED or } \\
\text { equivalent } \\
(n=759)\end{array}$ & 5.1 & 29.5 & 45.4 & 16.6 & 3.3 \\
\hline $\begin{array}{l}\text { Some college or } \\
\text { AA Degree } \\
(n=883)\end{array}$ & 12.4 & 32.6 & 39.4 & 13.0 & 2.6 \\
\hline $\begin{array}{l}\text { College graduate } \\
\text { or Above } \\
(n=659)\end{array}$ & 21.7 & 41.7 & 27.5 & 7.6 & 1.4 \\
\hline \multicolumn{6}{|l|}{ Marital Status } \\
\hline $\begin{array}{l}\text { Married } \\
(\mathrm{n}=1861)\end{array}$ & 11.8 & 34.3 & 36.7 & 13.9 & 3.3 \\
\hline $\begin{array}{l}\text { Widowed } \\
(n=758)\end{array}$ & 8.0 & 24.2 & 39.5 & 22.8 & 5.5 \\
\hline $\begin{array}{l}\text { Divorced } \\
(n=433)\end{array}$ & 17.0 & 26.1 & 37.4 & 17.1 & 2.4 \\
\hline $\begin{array}{l}\text { Separated } \\
(n=82)\end{array}$ & 5.5 & 15.8 & 39.8 & 29.1 & 9.8 \\
\hline $\begin{array}{l}\text { Never Married } \\
(n=169)\end{array}$ & 5.7 & 27.8 & 44.8 & 14.0 & 7.6 \\
\hline $\begin{array}{l}\text { Living with Partner } \\
(\mathrm{n}=85)\end{array}$ & 10.5 & 24.4 & 45.5 & 15.5 & 3.8 \\
\hline \multicolumn{6}{|l|}{ Living Alone } \\
\hline $\begin{array}{l}\text { Yes } \\
(n=857)\end{array}$ & 11.5 & 38.2 & 39.8 & 16.4 & 4.1 \\
\hline $\begin{array}{l}\text { No } \\
(n=2535)\end{array}$ & 11.3 & 31.5 & 37.3 & 16.1 & 3.8 \\
\hline
\end{tabular}


Table 4.2, continued

\begin{tabular}{cccccc}
\hline Characteristic & Excellent & $\begin{array}{l}\text { Very } \\
\text { Good }\end{array}$ & Good & Fair & Poor \\
\hline Poverty $^{\text {b }}$ & 5.0 & 18.2 & 37.9 & 29.3 & 9.6 \\
$\begin{array}{c}<130 \% \\
(n=920)\end{array}$ & 12.8 & 34.3 & 37.7 & 12.8 & 2.5 \\
$>130 \%$ & & & & \\
\hline
\end{tabular}

Data from NHANES 2009-2012 Demographics and Questionnaire Data Files

${ }^{a}$ All $n$ are analytic sample $\mathrm{n}$; all \% and mean data are weighted in order to represent the U.S. population. Weighting accounts for the probability of being selected for the sample, survey nonresponse, and differences between the sample and total U.S. population

${ }^{b} 130 \%$ of the Federal Poverty Level

\subsection{Diet Quality Predictor Variables: HEI and Self-Perceived Diet Quality}

Average total $\mathrm{HEI}$ scores calculated using the $\mathrm{NCI}$ population ratio method are shown in Table 4.3. The average mean total scores were similar for day one and day two.

Table 4.3 Average Mean Healthy Eating Index-2010 Total Scores Obtained Using the $\mathrm{NCl}$ Population Ratio Method

\begin{tabular}{lcc}
\hline & Mean & Standard Error \\
\hline Day 1 & 66.4 & 0.96 \\
Day 2 & 67.6 & 0.59 \\
\hline
\end{tabular}

Data from NHANES 2009-2012 Questionnaire Data Files

Table 4.4 shows the average $\mathrm{HEI}$ total scores calculated using the $\mathrm{NCl}$ population ratio method, categorized by response to the general health condition variable. Those who said their general health was excellent or very good had the highest average $\mathrm{HEI}$ total scores, and the lowest average $\mathrm{HEI}$ scores were for those who reported fair or poor HRQOL. Participants who had both days of dietary data were the only ones included for analysis involving actual diet quality $(n=3058)$. 
Table 4.4 Average Healthy Eating Index-2010 Total Scores and Standard Errors by Health-Related Quality of Life Category Using the NCl Population Ratio Method in Adults aged 60+ for the NHANES Survey Cycles 2009-2010 and 2011-2012

\begin{tabular}{cccccc}
\hline & $\begin{array}{c}\text { Excellent } \\
\overline{\mathbf{x}}(\mathrm{SE})\end{array}$ & $\begin{array}{c}\text { Very Good } \\
\overline{\mathbf{x}}(\mathrm{SE})\end{array}$ & $\begin{array}{c}\text { Good } \\
\overline{\mathbf{x}}(\mathrm{SE})\end{array}$ & $\begin{array}{c}\text { Fair } \\
\overline{\mathbf{x}}(\mathrm{SE})\end{array}$ & $\begin{array}{c}\text { Poor } \\
\mathbf{\mathbf { x }}(\mathrm{SE})\end{array}$ \\
\hline Day 1 & $72.9(3.6)$ & $68.1(1.0)$ & $64.3(0.9)$ & $58.7(1.2)$ & $59.4(1.7)$ \\
Day 2 & $70.1(2.3)$ & $70.1(1.1)$ & $66.6(1.1)$ & $61.5(1.4)$ & $58.1(2.6)$ \\
\hline \multicolumn{7}{l}{ Data from NHANES 2009-2012 Demographics, Questionnaire, and Dietary Data Files }
\end{tabular}

The average HEI total scores by self-perceived diet quality category for day one and day two are shown in Table 4.5. Older adults who reported their diet quality as excellent had the highest actual diet quality (HEI) total scores for day one and day two, and the actual average $\mathrm{HEI}$ scores were lower as self-perceived diet quality category was lower.

Table 4.5 Average Healthy Eating Index-2010 Total Scores by Self-Perceived Diet Quality Category Using the $\mathrm{NCl}$ Population Ratio Method from Older Adults 60+, NHANES Data 2009-2010 and 2011-2012

\begin{tabular}{llllll}
\hline & $\begin{array}{llll}\text { Excellent } \\
\overline{\mathbf{x}}(\mathrm{SE})\end{array}$ & $\begin{array}{l}\text { Very Good } \\
\overline{\mathbf{x}}(\mathrm{SE})\end{array}$ & $\begin{array}{l}\text { Good } \\
\overline{\mathbf{x}}(\mathrm{SE})\end{array}$ & $\begin{array}{l}\text { Fair } \\
\overline{\mathbf{x}}(\mathrm{SE})\end{array}$ & $\begin{array}{l}\text { Poor } \\
\overline{\mathbf{x}}(\mathrm{SE})\end{array}$ \\
\hline Day 1 & $76.3(2.7)$ & $69.6(1.2)$ & $61.8(0.9)$ & $58.0(1.6)$ & $56.2(3.2)$ \\
Day 2 & $70.6(1.7)$ & $69.7(0.9)$ & $66.9(1.1)$ & $58.8(1.2)$ & $56.9(2.1)$ \\
\hline
\end{tabular}

Data from NHANES 2009-2012 Demographics, Questionnaire, and Dietary Data Files

Figure 4.1 shows the correlation between the mean individual HEI total scores unweighted for day one and day two. The scatterplot shows a moderate positive correlation $\left(R^{2}=0.46\right)$ between the HEI total scores for days one and two. 


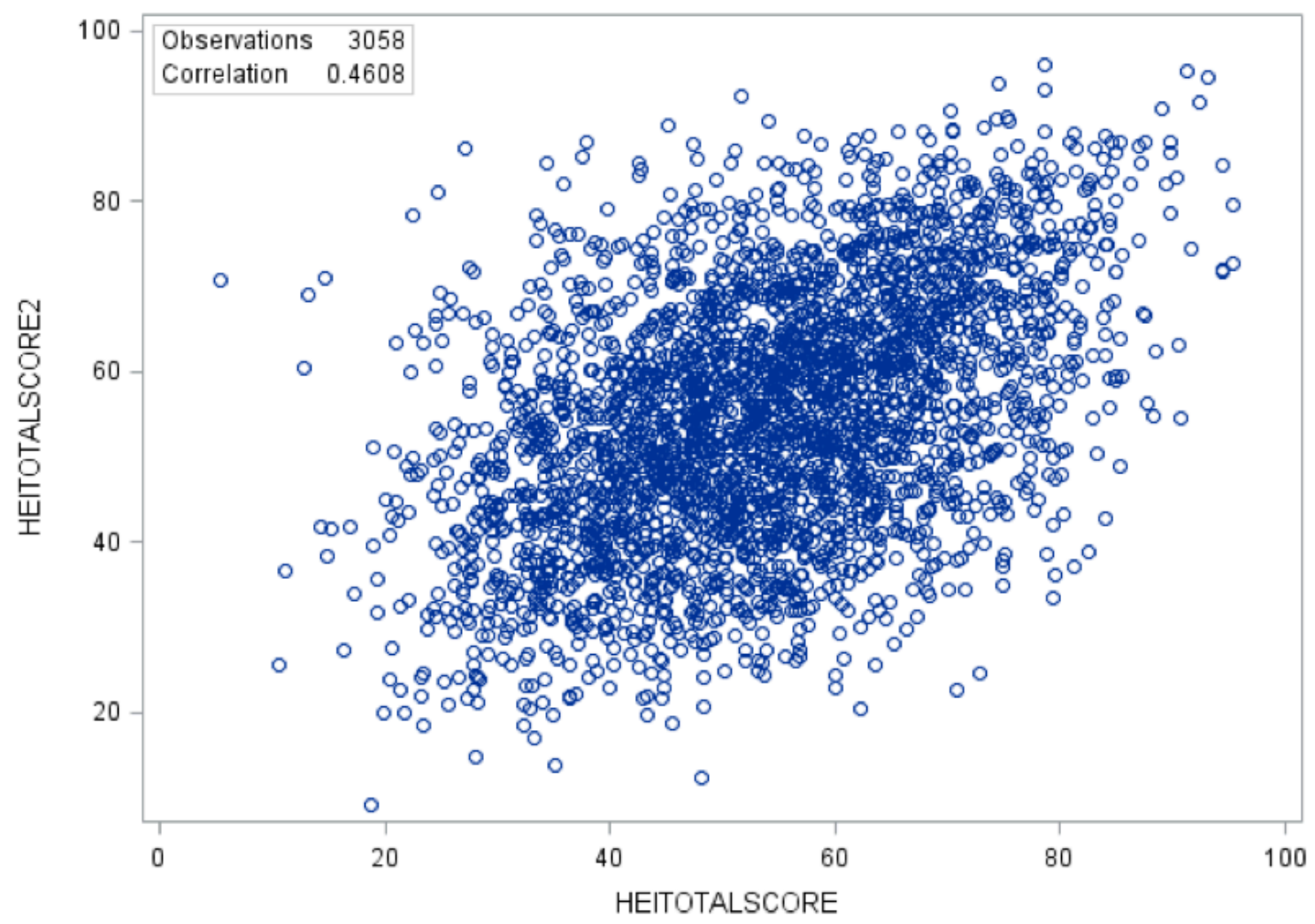

Figure 4.1 Scatterplot of the correlation between healthy eating index-2010 total scores for day 1 and day 2, calculated from NHANES dietary data, 2009-2010 and 2011-2012

Additionally, quintiles were calculated as shown in Table 4.6 . About $66 \%$ of people in quintile 1 of day 1 were in quintile 1 or 2 of day 2 , and about $70 \%$ of people in quintile 5 of day 1 were in quintile 4 or 5 of day 2 , showing some consistency of diet quality between days. At the same time, only about $6 \%$ had diet quality in opposite extremes for day 1 and day 2, i.e. quintile 1 of day 1 vs. quintile 5 of day 2 , or quintile 5 day 1 vs. quintile 1 day 2 . 
Table 4.6 Unweighted Percent of Subjects for Healthy Eating Index-2010 Scores Day 1 and Day 2 Quintiles from NHANES Dietary Data 2009-2010 and 2011-2012

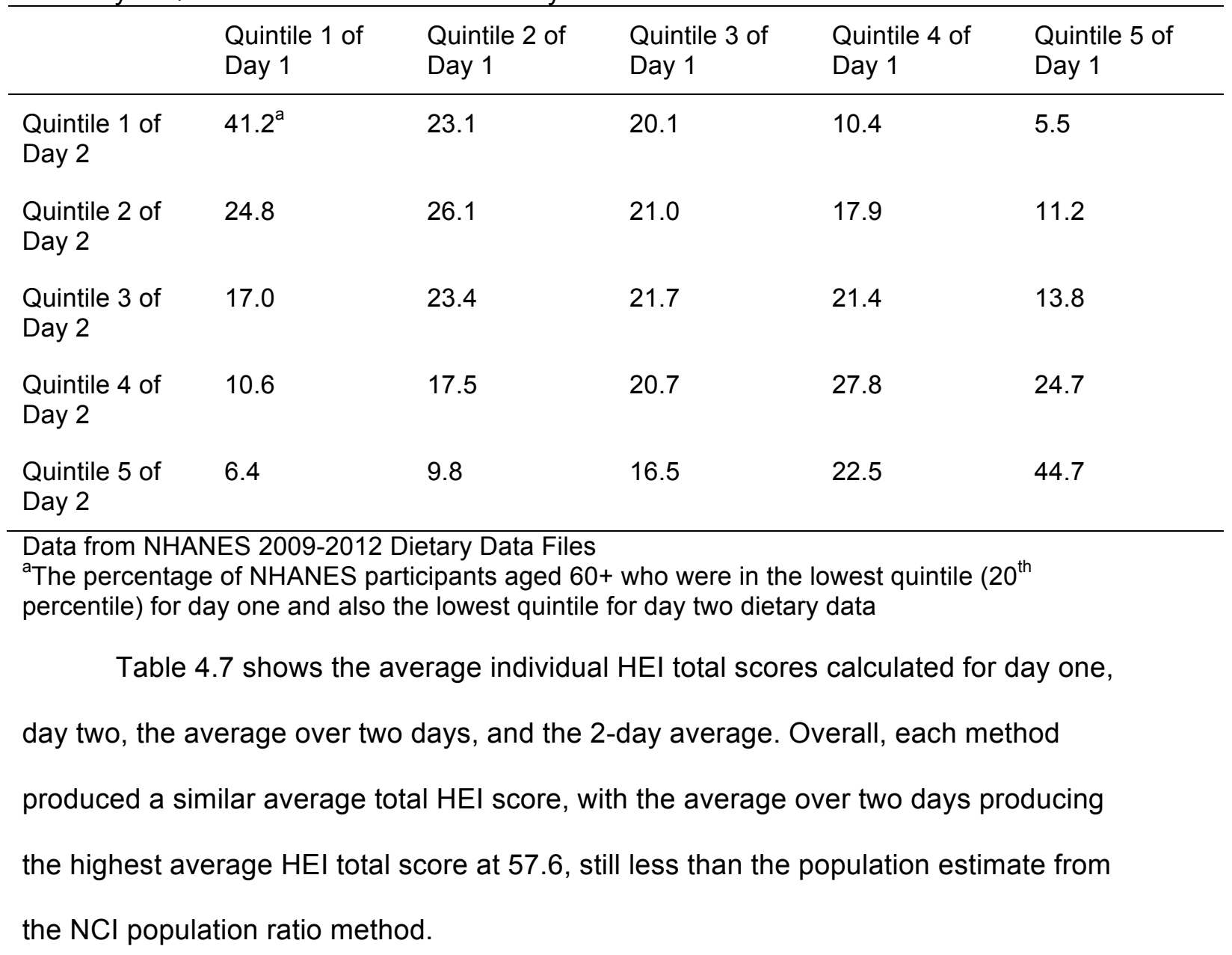

The weighted percentages of older adults aged 60+ in each self-perceived diet quality response category (excellent, very good, good, fair, and poor) by older adult age groups as well as the overall sample 60+ years are shown in Table 4.8. Most older adults $(40.8 \%)$ reported having good diet quality and very good diet quality $(28.6 \%)$. Overall, older adults aged $80+$ had the highest percentage of "excellent" diet quality responses (18.1\%) compared to the other four diet quality response categories. Adults aged $60-69$ years had the highest percentage in the fair diet quality category $(14.8 \%)$, while adults aged $70-79$ years had the highest percentage in the very good diet quality category $(33.5 \%)$. 
Table 4.7 Individual Average Daily Healthy Eating Index-2010 Scores, Average Over Two Days, and 2-Day Average Healthy Eating Index-2010 Total Scores of Older Adults 60+ from NHANES Data 2009-2010 and 2011-2012

\begin{tabular}{|c|c|c|c|c|c|c|c|}
\hline & Mean & SE & Min & Max & $\begin{array}{c}\text { 25th } \\
\text { percentile }\end{array}$ & $\begin{array}{c}\text { 75th } \\
\text { percentile }\end{array}$ & $95 \% \mathrm{Cl}$ \\
\hline Day 1 & 54.8 & 0.57 & 5.5 & 95.4 & 44.0 & 65.8 & $53.6,55.9$ \\
\hline Day 2 & 55.7 & 0.42 & 9.1 & 96.1 & 45.0 & 66.1 & $54.8,56.5$ \\
\hline $\begin{array}{l}\text { Average }^{a} \\
\text { Over } 2 \\
\text { Days }\end{array}$ & 57.6 & 0.62 & 14.3 & 95.0 & 48.5 & 67.4 & $56.4,58.9$ \\
\hline $\begin{array}{l}\text { 2-Day } \\
\text { Average }^{b}\end{array}$ & 55.0 & 0.52 & 13.9 & 93.8 & 46.5 & 63.2 & $53.9,56.0$ \\
\hline \multicolumn{8}{|c|}{$\begin{array}{l}\text { Data from NHANES 2009-2012 Demographics, Questionnaire, and Dietary Data Files } \\
\text { a Calculated by adding each food intake variable for day } 1 \text { and day } 2 \text { together before applying the } \\
\text { legume allocation and HEI scoring algorithm. The HEI components were then added together to } \\
\text { obtain the average score over two days } \\
\text { b Calculated by adding each HEI component score for day one and day two together, dividing by } \\
\text { two, and adding those component scores together for the total HEI score }\end{array}$} \\
\hline
\end{tabular}

Table 4.8 Self-Perceived Diet Quality Weighted Percentages of Older Adults 60+ by Age Groups

Excellent Very Good Good Fair Poor

\begin{tabular}{|c|c|c|c|c|c|}
\hline $\begin{array}{c}\text { All Subjects } \\
n^{a}=3690\end{array}$ & 15.1 & 28.6 & 40.8 & 12.5 & 3.1 \\
\hline $\begin{array}{c}\text { 60-69 years } \\
n=1814\end{array}$ & 14.9 & 24.9 & 41.8 & 14.8 & 3.6 \\
\hline $\begin{array}{c}\text { 70-79 years } \\
n=1155\end{array}$ & 13.7 & 33.5 & 39.6 & 10.5 & 2.8 \\
\hline $\begin{array}{c}80+\text { years } \\
n=721\end{array}$ & 18.1 & 30.9 & 39.8 & 9.3 & 2.0 \\
\hline
\end{tabular}

Data from NHANES 2009-2012 Questionnaire Data Files

${ }^{a}$ All $n$ are analytic sample $n$; all data are weighted in order to represent the U.S. population. Weighting accounts for the probability of being selected for the sample, survey nonresponse, and differences between the sample and total U.S. population

Table 4.9 shows the average individual HEI total scores over two days by selfperceived diet quality response category. Older adults who self-reported their diet as "excellent" had the highest HEI total score, and HEI scores were lower as self-perceived diet quality was lower. 
Table 4.9 Average Individual Healthy Eating Index-2010 Scores Over Two Days by SelfPerceived Diet Quality Response Category

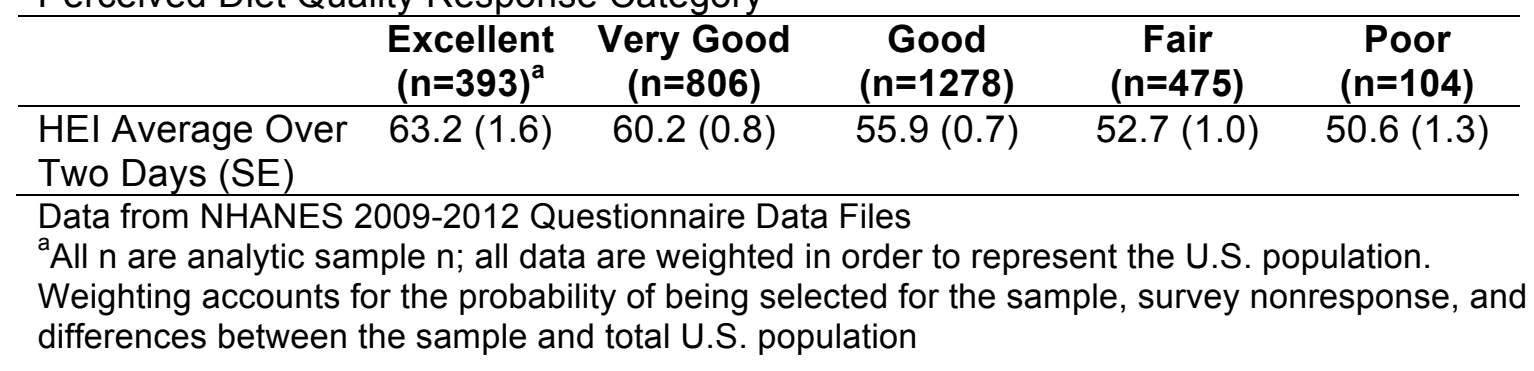

\subsection{Other Diet-Related Predictor Variable Data}

Table 4.10 shows weighted percentages of other nutrition-related predictor variables by age groups used for this thesis. For the community/government meals delivered variable, the percentage of older adults who had meals delivered was lowest in the $60-69$ age group (1.3\%), and highest in the $80+$ age group $(7.2 \%)$. The percentage of older adults who said they did not eat meals at a community or senior center was highest among the $60-69$ year olds (95.9\%) and lower in the $70-79$ year olds $(92.5 \%)$ and in the $80+$ year olds $(89.2 \%)$. For the variable regarding difficulty with meal prep, the percentage that responded with "no difficulty" was highest in the 60-69 age group $(89.4 \%)$ followed by the $70-79$ age group $(86.6 \%)$ and the $80+$ age group $(76.2 \%)$. However, $3.9 \%$ of $60-69$ year olds, $6.1 \%$ of $70-79$ year olds, and $9.4 \%$ of $80+$ year olds did not do their own meal prep, and $6.4 \%$ of $80+$ year olds were unable to do this activity. For the using fork/knife/drinking from a cup variable, $95.1 \%$ of $60-69$ year olds, $95.0 \%$ of $70-79$ year olds, and $89.6 \%$ of $80+$ year olds responded with "no difficulty." For the poor appetite/overeating variable, participants who responded "not at all" bothered was highest in the $80+$ age group (87.9\%). 
Table 4.10 Weighted Percentages of Other Diet-Related Predictor Variables of Older Adults 60+ by Age Group from NHANES Data Cycles 2009-2010 and 2011-2012

\begin{tabular}{|c|c|c|}
\hline $\begin{array}{c}60-69 \text { years } \\
n=1815^{\mathrm{a}}\end{array}$ & $\begin{array}{c}70-79 \\
\text { years } \\
n=1155\end{array}$ & $\begin{array}{c}80+ \\
\text { years } \\
n=722\end{array}$ \\
\hline
\end{tabular}

\section{Community/Government Meals Delivered}
Yes
0.8
2.9
7.4
2.6

Eat Meals at Communityl

Senior Center
Yes
3.9
6.6
11.5
6.1

Meal Prep Difficulty

No Difficulty

92.7

89.6

78.7

89.3

Some Difficulty

3.4

3.9

6.0

4.0

Much Difficulty

0.6

0.6

2.0

0.8

Unable to Do

0.7

1.2

6.0

1.8

Don't Do

2.7

4.6

7.2

4.1

\section{Using Fork/Knife/Drinking \\ From a Cup Difficulty}

$\begin{array}{lcccc}\text { No Difficulty } & 96.9 & 95.5 & 89.9 & 95.2 \\ \text { Some Difficulty } & 2.5 & 3.3 & 8.0 & 3.7 \\ \text { Much Difficulty } & 0.5 & 0.9 & 1.0 & 0.7 \\ \text { Unable to Do } & 0.2 & 0.3 & 0.8 & 0.3 \\ \text { Don't Do } & 0 & 0 & 0.2 & 0\end{array}$

\section{Bothered by Poor} Appetite/Overeating

\begin{tabular}{|c|c|c|c|c|}
\hline Not at All & 82.0 & 84.7 & 86.9 & 83.6 \\
\hline Several Days & 10.7 & 9.5 & 7.7 & 9.9 \\
\hline More than Half the Days & 3.8 & 2.2 & 1.6 & 3.1 \\
\hline Nearly Every Day & 3.4 & 3.0 & 3.7 & 3.3 \\
\hline
\end{tabular}




\subsection{Regression Model Data}

Table 4.11 shows the odds ratios, $95 \%$ confidence intervals, and p-values of the $\mathrm{HEI}$ and self-perceived diet quality models for the overall HRQOL outcome predicting the binary outcome of good or better HRQOL (vs. fair/poor HRQOL). Univariate analyses showed that all variables except gender and living alone were significant predictors of good/better HRQOL, though these were kept in the model because they are important demographics of the older adult population. Results of multivariate analyses, in Table 4.11, indicated that the HEl average individual total scores over two days variable was predictive of good or better HRQOL $(p<0.0001)$. Specifically, a one-unit increase in HEI was associated with $3 \%$ greater odds of better HRQOL. The HEI variable was also significant when the average scores were separated into quartiles $(p=0.0004, p=0.0082$, $\mathrm{p}<0.0001$ ) for the $2^{\text {nd }}, 3^{\text {rd }}$, and $4^{\text {th }}$ quartiles compared with the lowest quartile of HEI. The average $\mathrm{HEI}$ scores for the 4 quartiles were $31.4,52.7,62.2$, and 81.3 . Those in the $4^{\text {th }}$ quartile for diet quality had 2.35 times greater odds of having good or better HRQOL compared to the $1^{\text {st }}$ quartile for diet quality $(p<0.0001)$. For the self-perceived diet quality variable, those who reported good or better diet quality had 3.12 times greater odds of good or better HRQOL compared to those who reported poor diet quality $(p<0.0001)$. The variables age, gender, marital status, living alone, and meals eaten at a senior center did not significantly predict HRQOL. For the meals delivered variable in the Continuous HEl Model, those who said they had community/government meals delivered in the last 12 months of being surveyed had $64 \%$ lower odds of good/better HRQOL compared to those who did not have any meals delivered $(p=0.0044)$. Participants who stated they had difficulty with meal prep had $79 \%$ lower odds of good/better HRQOL compared to those who reported no difficulty $(p<0.0001)$. For the poor appetite/overeating variable, those who reported that they were bothered by poor appetite/overeating nearly every day in the past two weeks had $82 \%$ lower odds of 
good/better HRQOL compared to those who were not bothered at all $(p<0.0001)$. Model 1 where the HEI was kept as a continuous variable, those with a college education were 2.72 times more likely to have good/better HRQOL compared to those with less than a $9^{\text {th }}$ grade education $(p<0.0001)$. 
Table 4.11 Adjusted Odds Ratios for the Continuous HEI Model, the Categorical HEI Model, and the Self-Perceived Diet Quality Model Predicting Good or Better HRQOL ${ }^{\text {ab }}$

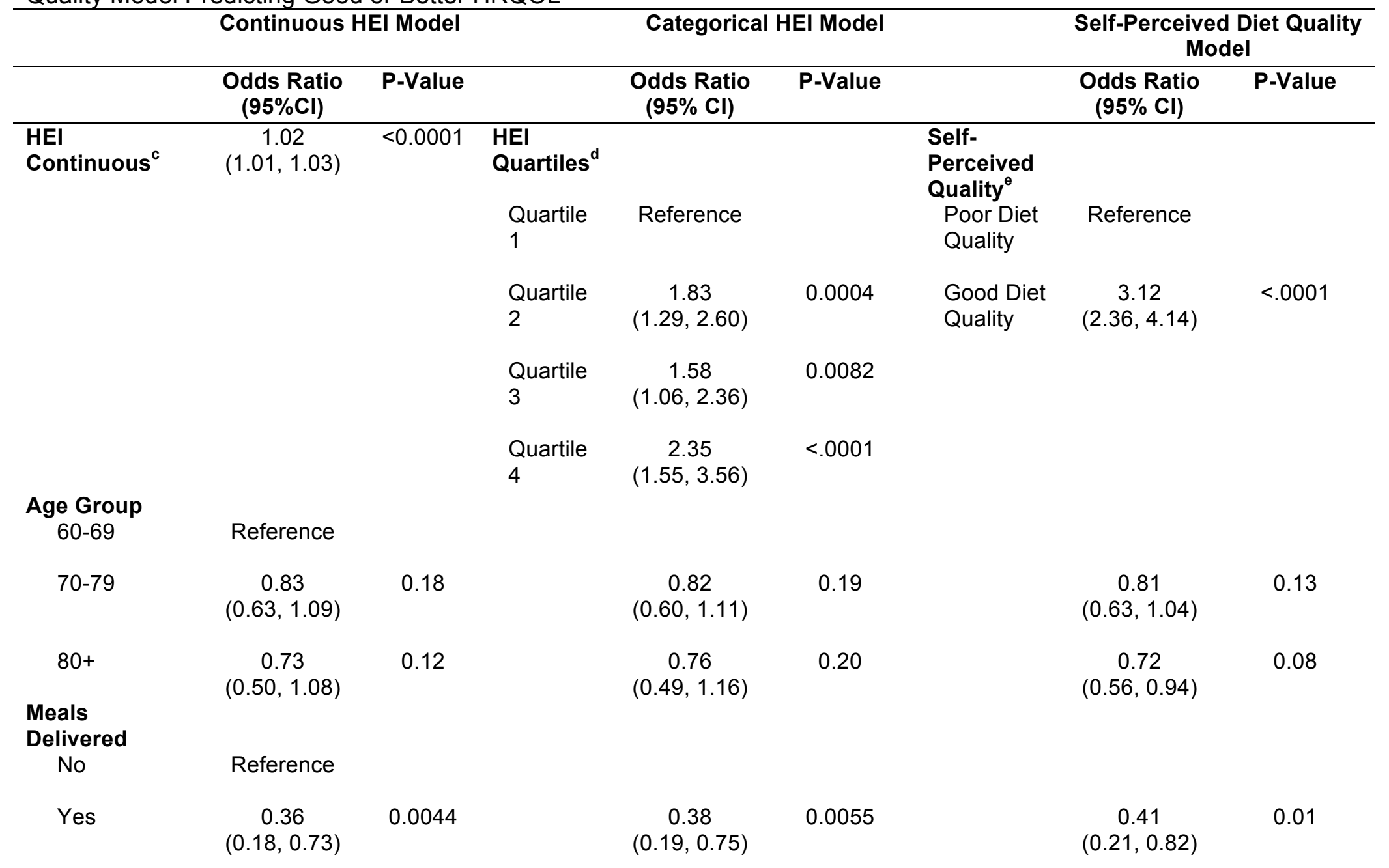


Table 4.11, continued

\begin{tabular}{|c|c|c|c|c|c|c|}
\hline & \multicolumn{2}{|c|}{ Continuous HEI Model } & \multicolumn{2}{|c|}{ Categorical HEI Model } & \multicolumn{2}{|c|}{$\begin{array}{c}\text { Self-Perceived Diet } \\
\text { Quality Model }\end{array}$} \\
\hline & $\begin{array}{l}\text { Odds Ratio } \\
(95 \% \mathrm{Cl})\end{array}$ & P-Value & $\begin{array}{l}\text { Odds Ratio } \\
(95 \% \mathrm{Cl})\end{array}$ & P-Value & $\begin{array}{l}\text { Odds Ratio } \\
(95 \% \mathrm{Cl})\end{array}$ & P-Value \\
\hline $\begin{array}{l}\text { Meals Eaten at } \\
\text { Senior Center } \\
\text { No }\end{array}$ & Reference & & & & & \\
\hline Yes & $\begin{array}{c}0.86 \\
(0.45,1.52)\end{array}$ & 0.59 & $\begin{array}{c}0.88 \\
(0.48,1.59)\end{array}$ & 0.66 & $\begin{array}{c}0.87 \\
(0.58,1.32)\end{array}$ & 0.51 \\
\hline $\begin{array}{l}\text { Meal Prep } \\
\text { Difficulty }\end{array}$ & & & & & & \\
\hline No Difficulty & Reference & & & & & \\
\hline Difficulty & $\begin{array}{c}0.21 \\
(0.12,0.36)\end{array}$ & $<.0001$ & $\begin{array}{c}0.21 \\
(0.12,0.37)\end{array}$ & $<.0001$ & $\begin{array}{c}0.23 \\
(0.15,0.36)\end{array}$ & $<.0001$ \\
\hline $\begin{array}{l}\text { Don't Do } \\
\text { This } \\
\text { Activity }\end{array}$ & $\begin{array}{c}0.59 \\
(0.28,1.24)\end{array}$ & 0.16 & $\begin{array}{c}0.58 \\
(0.27,1.24)\end{array}$ & 0.16 & $\begin{array}{c}0.51 \\
(0.30,0.88)\end{array}$ & 0.02 \\
\hline
\end{tabular}




\begin{tabular}{|c|c|c|c|c|c|c|}
\hline & \multicolumn{2}{|c|}{ Continuous HEI Model } & \multicolumn{2}{|c|}{ Categorical HEI Model } & \multicolumn{2}{|c|}{$\begin{array}{c}\text { Self-Perceived Diet } \\
\text { Quality Model }\end{array}$} \\
\hline & $\begin{array}{c}\text { Odds Ratio } \\
(95 \% \mathrm{Cl})\end{array}$ & P-Value & $\begin{array}{c}\text { Odds Ratio } \\
(95 \% \mathrm{Cl})\end{array}$ & P-Value & $\begin{array}{c}\text { Odds Ratio } \\
(95 \% \mathrm{Cl})\end{array}$ & P-Value \\
\hline $\begin{array}{l}\text { Bothered by } \\
\text { Poor Appetite/ } \\
\text { Overeating } \\
\text { Not at All }\end{array}$ & Reference & & & & & \\
\hline $\begin{array}{l}\text { Several } \\
\text { Days }\end{array}$ & $\begin{array}{c}0.43 \\
(0.28,0.66)\end{array}$ & 0.0001 & $\begin{array}{c}0.42 \\
(0.27,0.65)\end{array}$ & 0.0001 & $\begin{array}{c}0.61 \\
(0.43,0.86)\end{array}$ & 0.0043 \\
\hline $\begin{array}{l}\text { More Than } \\
\text { Half the } \\
\text { Days }\end{array}$ & $\begin{array}{c}0.20 \\
(0.10,0.40)\end{array}$ & $<.0001$ & $\begin{array}{c}0.18 \\
(0.09,0.36)\end{array}$ & $<.0001$ & $\begin{array}{c}0.38 \\
(0.21,0.68)\end{array}$ & 0.0012 \\
\hline $\begin{array}{l}\text { Nearly } \\
\text { Every Day }\end{array}$ & $\begin{array}{c}0.18 \\
(0.10,0.32)\end{array}$ & $<.0001$ & $\begin{array}{c}0.16 \\
(0.09,0.30)\end{array}$ & $<.0001$ & $\begin{array}{c}0.24 \\
(0.14,0.44)\end{array}$ & $<.0001$ \\
\hline $\begin{array}{r}\text { Gender } \\
\text { Male }\end{array}$ & Reference & & & & & \\
\hline Female & $\begin{array}{c}1.22 \\
(0.89,1.68)\end{array}$ & 0.23 & $\begin{array}{c}1.20 \\
(0.87,1.65)\end{array}$ & 0.27 & $\begin{array}{c}1.24 \\
(0.92,1.68)\end{array}$ & 0.16 \\
\hline $\begin{array}{l}\text { Marital Status } \\
\text { Married }\end{array}$ & Reference & & & & & \\
\hline Not Married & $\begin{array}{c}0.90 \\
(0.62,1.29)\end{array}$ & 0.56 & $\begin{array}{c}0.93 \\
(0.63,1.39)\end{array}$ & 0.73 & $\begin{array}{c}0.93 \\
(0.65,1.31)\end{array}$ & 0.66 \\
\hline
\end{tabular}


Table 4.11, continued

\begin{tabular}{|c|c|c|c|c|c|c|}
\hline & \multicolumn{2}{|c|}{ Continuous HEI Model } & \multicolumn{2}{|c|}{ Categorical HEI Model } & \multicolumn{2}{|c|}{$\begin{array}{c}\text { Self-Perceived Diet } \\
\text { Quality Model }\end{array}$} \\
\hline & $\begin{array}{c}\text { Odds Ratio } \\
(95 \% \mathrm{Cl})\end{array}$ & P-Value & $\begin{array}{l}\text { Odds Ratio } \\
(95 \% \mathrm{Cl})\end{array}$ & P-Value & $\begin{array}{l}\text { Odds Ratio } \\
\text { (95\% Cl) }\end{array}$ & P-Value \\
\hline $\begin{array}{c}\text { Education } \\
<9^{\text {th }} \\
\end{array}$ & Reference & & & & & \\
\hline $\begin{array}{l}\text { Grade } \\
9^{\text {th }}-11^{\text {th }} \\
\text { Grade }\end{array}$ & $\begin{array}{c}1.16 \\
(0.75,1.79)\end{array}$ & 0.51 & $\begin{array}{c}1.14 \\
(0.74,1.75)\end{array}$ & 0.56 & $\begin{array}{c}1.47 \\
(0.95,2.28)\end{array}$ & 0.08 \\
\hline $\begin{array}{l}\text { High School } \\
\text { Graduate }\end{array}$ & $\begin{array}{c}1.95 \\
(1.32,2.87)\end{array}$ & 0.0008 & $\begin{array}{c}1.96 \\
(1.32,2.92)\end{array}$ & 0.0008 & $\begin{array}{c}2.31 \\
(1.61,3.31)\end{array}$ & $<.0001$ \\
\hline $\begin{array}{l}\text { Some } \\
\text { College/AA } \\
\text { Degree }\end{array}$ & $\begin{array}{c}2.63 \\
(1.72,4.04)\end{array}$ & $<.0001$ & $\begin{array}{c}2.61 \\
(1.71,3.99)\end{array}$ & $<.0001$ & $\begin{array}{c}2.77 \\
(1.88,4.09)\end{array}$ & $<.0001$ \\
\hline $\begin{array}{l}\text { College } \\
\text { Graduate or } \\
\text { Above }\end{array}$ & $\begin{array}{c}2.72 \\
(1.68,4.43)\end{array}$ & $<.0001$ & $\begin{array}{c}2.78 \\
(1.74,4.46)\end{array}$ & $<.0001$ & $\begin{array}{c}3.89 \\
(2.65,5.70)\end{array}$ & $<.0001$ \\
\hline $\begin{array}{l}\text { Poverty } \\
\quad>130 \%\end{array}$ & Reference & & & & & \\
\hline$<=130 \%$ & $\begin{array}{c}0.67 \\
(0.43,1.04)\end{array}$ & 0.08 & $\begin{array}{c}0.66 \\
(0.42,1.02)\end{array}$ & 0.06 & $\begin{array}{c}0.62 \\
(0.45,0.85)\end{array}$ & 0.0030 \\
\hline
\end{tabular}


Table 4.11, continued

\begin{tabular}{|c|c|c|c|c|c|c|}
\hline & \multicolumn{2}{|c|}{ Continuous HEI Model } & \multicolumn{2}{|c|}{ Categorical HEI Model } & \multicolumn{2}{|c|}{$\begin{array}{c}\text { Self-Perceived Diet } \\
\text { Quality Model }\end{array}$} \\
\hline & $\begin{array}{c}\text { Odds Ratio } \\
(95 \% \mathrm{Cl})\end{array}$ & P-Value & $\begin{array}{l}\text { Odds Ratio } \\
(95 \% \mathrm{Cl})\end{array}$ & P-Value & $\begin{array}{c}\text { Odds Ratio } \\
(95 \% \mathrm{Cl})\end{array}$ & P-Value \\
\hline $\begin{array}{l}\text { Race/Ethnicity } \\
\text { Non- } \\
\text { Hispanic } \\
\text { White }\end{array}$ & Reference & & & & & \\
\hline $\begin{array}{l}\text { Mexican- } \\
\text { American }\end{array}$ & $\begin{array}{c}0.24 \\
(0.13,0.41)\end{array}$ & $<.0001$ & $\begin{array}{c}0.24 \\
(0.13,0.42)\end{array}$ & $<.0001$ & $\begin{array}{c}0.37 \\
(0.27,0.53)\end{array}$ & .0001 \\
\hline $\begin{array}{l}\text { Non- } \\
\text { Hispanic } \\
\text { Black }\end{array}$ & $\begin{array}{c}0.51 \\
(0.39,0.67)\end{array}$ & $<.0001$ & $\begin{array}{c}0.52 \\
(0.40,0.67)\end{array}$ & $<.0001$ & $\begin{array}{c}0.52 \\
(0.42,0.65)\end{array}$ & $<.0001$ \\
\hline $\begin{array}{l}\text { Other } \\
\text { Hispanic }\end{array}$ & $\begin{array}{c}0.33 \\
(0.18,0.58)\end{array}$ & 0.0002 & $\begin{array}{c}0.33 \\
(0.19,0.58)\end{array}$ & 0.0001 & $\begin{array}{c}0.46 \\
(0.31,0.67)\end{array}$ & $<.0001$ \\
\hline $\begin{array}{l}\text { Other/ } \\
\text { Multicultural }\end{array}$ & $\begin{array}{c}0.87 \\
(0.37,2.00)\end{array}$ & 0.74 & $\begin{array}{c}0.92 \\
(0.38,2.19)\end{array}$ & 0.84 & $\begin{array}{c}0.82 \\
(0.43,1.58)\end{array}$ & 0.56 \\
\hline $\begin{array}{l}\text { Live Alone } \\
\quad \text { No }\end{array}$ & Reference & & & & & \\
\hline Yes & $\begin{array}{c}1.25 \\
(0.83,1.89)\end{array}$ & 0.29 & $\begin{array}{c}1.21 \\
(0.80,1.83)\end{array}$ & 0.38 & $\begin{array}{c}1.20 \\
(0.80,1.79)\end{array}$ & 0.37 \\
\hline
\end{tabular}

${ }^{a}$ Odds ratios when all variables are included in the model

${ }^{b}$ Data from NHANES 2009-2012 Demographics and Questionnaire Data Files; all data are weighted in order to represent the U.S.

population. Weighting accounts for the probability of being selected for the sample, survey nonresponse, and differences between the sample and total U.S. population

${ }^{\mathrm{C}} \mathrm{HEl}$ (Healthy Eating index) individual total average scores over day 1 and day 2 of NHANES dietary data

${ }^{\mathrm{d}} \mathrm{HEI}$ (Healthy Eating Index) individual total average scores over day 1 and day 2 of NHANES dietary data separated by quartiles

"Self-perceived diet quality variable asked how the participants perceived their health by responding with either "excellent," "very good," "good," "fair," and "poor." Categories were collapsed into poor diet quality ("fair" and "poor" responses) and good or better diet quality ("excellent," "very good," and "good" responses) 
The interaction between $\mathrm{HEI}$ and meals eaten at a senior center $(p=0.0002)$, as well as $\mathrm{HEI}$ and poverty (0.0004) were significant in the Continuous HEI model with overall HRQOL as the outcome (data not shown). No other interactions between HEI and other variables were statistically significant.

Table 4.12 shows the odds ratios for the HEI variable in older adults who ate meals at a senior center vs. those who did not eat meals at a senior center (models run separately), and for those who were in poverty (<=130\%) vs. those who were not in poverty (>130\%) (models run separately). HEI was a significant predictor of HRQOL only in those who did not eat at a senior center and in those who were not in poverty $(>130 \%)$. HEI was a marginally significant $(p=0.03)$ predictor of HRQOL in those who ate at a senior center.

Table 4.12 Adjusted Odds Ratios of Healthy Eating Index-2010 Average Total Scores Over Two Days by Meals Eaten and Poverty Responses ${ }^{a}$

HEl Continuous

\begin{tabular}{|c|c|c|}
\hline & $\begin{array}{c}\text { OR } \\
(95 \% \mathrm{Cl}) \\
\end{array}$ & P-value \\
\hline \multicolumn{3}{|c|}{$\begin{array}{l}\text { Meals Eaten at Senior } \\
\text { Center Model }^{b}\end{array}$} \\
\hline $\begin{array}{l}\text { Yes } \\
(n=182)\end{array}$ & $\begin{array}{c}0.95 \\
(0.92,0.99)\end{array}$ & 0.03 \\
\hline $\begin{array}{l}\text { No } \\
(n=2874)\end{array}$ & $\begin{array}{c}1.03 \\
(1.02,1.04)\end{array}$ & $<0.0001$ \\
\hline \multicolumn{3}{|c|}{ Poverty Model $^{c}$} \\
\hline $\begin{array}{l}<=130 \% \\
(n=783)\end{array}$ & $\begin{array}{c}0.99 \\
(0.98,1.01)\end{array}$ & 0.35 \\
\hline $\begin{array}{l}>130 \% \\
(n=2003)\end{array}$ & $\begin{array}{c}1.04 \\
(1.02,1.05)\end{array}$ & $<0.0001$ \\
\hline \multicolumn{3}{|c|}{$\begin{array}{l}\text { Data from NHANES 2009-2012 Demographics and Questionnaire Data Files; all data are } \\
\text { weighted in order to represent the U.S. population. Weighting accounts for the probability of } \\
\text { selected for the sample, survey nonresponse, and differences between the sample and tota } \\
\text { population } \\
{ }^{2} \text { Adjusted for poverty, meals delivered, meal prep difficulty, poor appetite/overeating, age, } \\
\text { gender, marital status, education, race/ethnicity, and living alone } \\
{ }^{c} \text { Adjusted for meals eaten at senior center, meals delivered, meal prep difficulty, poor } \\
\text { appetite/overeating, age, gender, marital status, education, race/ethnicity, and living alone }\end{array}$} \\
\hline
\end{tabular}


Table 4.13 shows the adjusted odds ratios, $95 \%$ confidence intervals, and $p$ values for the models with the HEI continuous variable predicting the three healthy days outcomes physically unhealthy days, mentally unhealthy days, and inactive days due to physical/mental health. The logistic regression model is predicting a low number of unhealthy days (0-15 days) for each outcome variable vs.16 days or more. The same nutrition-related covariates (poor appetite/overeating, meal prep difficulty, government meal utilization) and the other covariates (age, gender, marital status, education, poverty, living alone) as the models with the overall HRQOL outcome were used with the other three outcome variables. The HEI continuous variable was not a significant predictor in any of the models predicting physically unhealthy days, mentally unhealthy days, and inactive days $(p=0.59, p=0.55, p=0.09)$.

Significant predictors of less physically unhealthy days were meals eaten at a senior center, difficulty with meal prep, and bothered by poor appetite/overeating. Older adults who reported eating at a senior center in the past 12 months had 1.9 times greater odds of fewer physically unhealthy days compared to those who did not report eating at a senior center $(p=0.02)$. Those who had difficulty with meal prep had $70 \%$ lower odds of having less physically unhealthy days, compared to those who had no difficulty with meal prep $(p<0.0001)$. Compared to those who were not bothered at all by poor appetite/overeating, those who were bothered by poor appetite/overeating nearly every day had $81 \%$ lower odds of having less physically unhealthy days $(p<0.0001)$.

For the Mentally Unhealthy Days Model, age, difficulty with meal prep, and bothered by poor appetite/overeating were significant predictors. Compared to 60-69 year olds, 70-79 year olds had 2.45 times and $80+$ year olds had 1.73 times greater odds of fewer mentally unhealthy days $(p=0.0027, p=0.04)$. 
Table 4.13 Adjusted Odds Ratios for the Continuous HEI Model Predicting Physically Unhealthy Days, Mentally Unhealthy Days, and Inactive Days due to Physical/Mental Health Issues ${ }^{\text {abc }}$

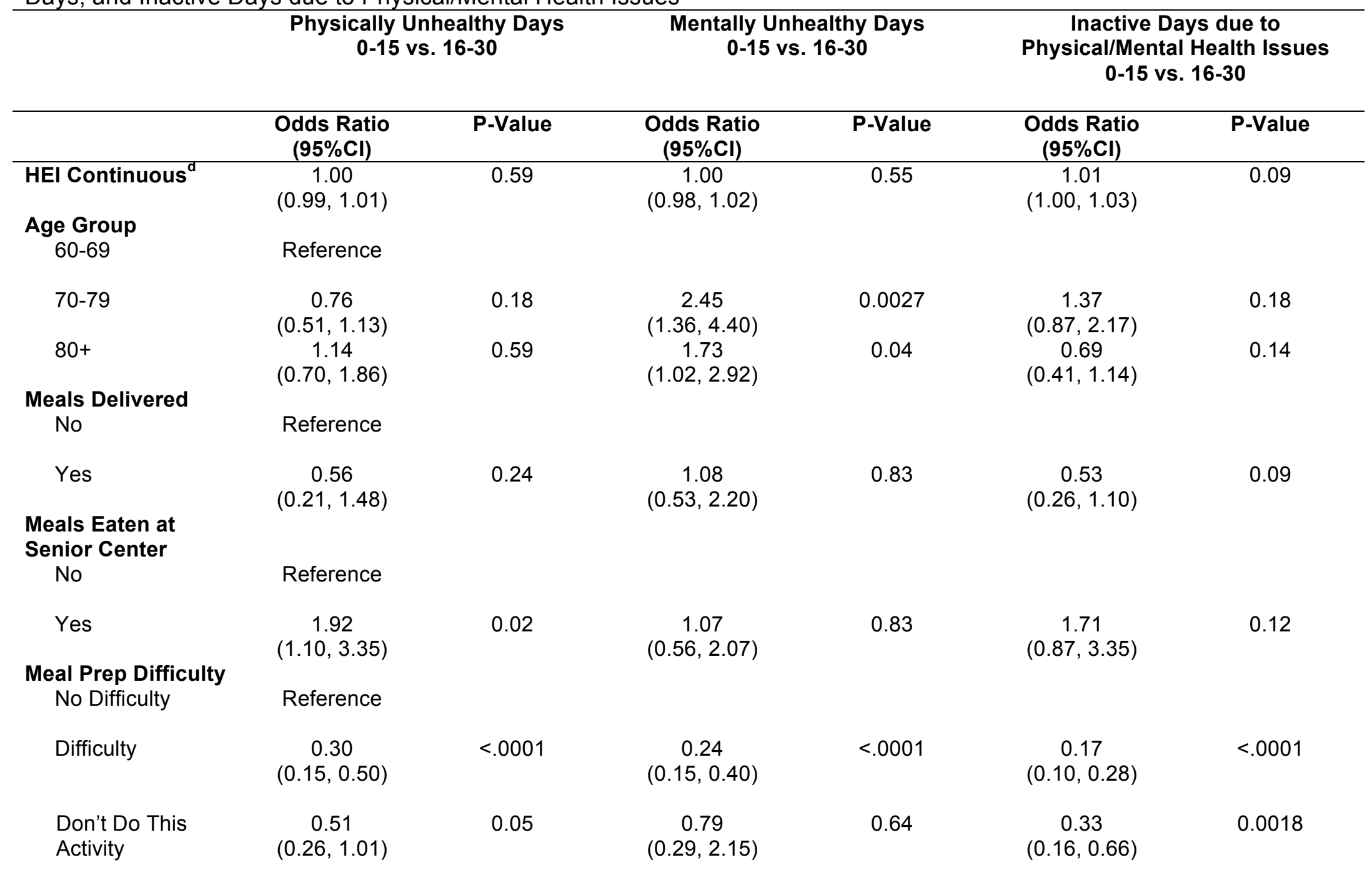




$$
\begin{array}{ccc}
\text { Physically Unhealthy Days } & \text { Mentally Unhealthy Days } & \text { Inactive Days due to } \\
0-15 \text { vs. } 16-30 & 0-15 \text { vs. } 16-30 & \text { Physical/Mental Health } \\
& & 0-15 \text { vs. 16-30 }
\end{array}
$$

\begin{tabular}{|c|c|c|c|c|c|c|}
\hline $\begin{array}{l}\text { Bothered by Poor } \\
\text { Appetite/Overeating }\end{array}$ & $\begin{array}{c}\text { Odds Ratio } \\
(95 \% \mathrm{Cl})\end{array}$ & P-Value & $\begin{array}{l}\text { Odds Ratio } \\
(95 \% \mathrm{Cl})\end{array}$ & P-Value & $\begin{array}{l}\text { Odds Ratio } \\
\text { (95\% Cl) }\end{array}$ & P-Value \\
\hline Not at All & Reference & & & & & \\
\hline Several Days & $\begin{array}{c}0.40 \\
(0.27,0.59)\end{array}$ & $<.0001$ & $\begin{array}{c}0.48 \\
(0.28,0.83)\end{array}$ & 0.0088 & $\begin{array}{c}0.34 \\
(0.18,0.64)\end{array}$ & 0.0008 \\
\hline $\begin{array}{l}\text { More Than Half } \\
\text { the Days }\end{array}$ & $\begin{array}{c}0.20 \\
(0.11,0.36)\end{array}$ & $<.0001$ & $\begin{array}{c}0.23 \\
(0.12,0.43)\end{array}$ & $<.0001$ & $\begin{array}{c}0.16 \\
(0.06,0.38)\end{array}$ & $<.0001$ \\
\hline Nearly Every Day & $\begin{array}{c}0.19 \\
(0.10,0.36)\end{array}$ & $<.0001$ & $\begin{array}{c}0.12 \\
(0.07,0.23)\end{array}$ & $<.0001$ & $\begin{array}{c}0.11 \\
(0.05,0.23)\end{array}$ & $<.0001$ \\
\hline $\begin{array}{r}\text { Gender } \\
\text { Male }\end{array}$ & Reference & & & & & \\
\hline Female & $\begin{array}{c}0.99 \\
(0.67,1.48)\end{array}$ & 0.98 & $\begin{array}{c}0.82 \\
(0.47,1.07)\end{array}$ & 0.48 & $\begin{array}{c}1.42 \\
(0.83,2.45)\end{array}$ & 0.20 \\
\hline $\begin{array}{l}\text { Marital Status } \\
\text { Married }\end{array}$ & Reference & & & & & \\
\hline Not Married & $\begin{array}{c}0.74 \\
(0.45,1.22)\end{array}$ & 0.24 & $\begin{array}{c}0.67 \\
(0.42,1.07)\end{array}$ & 0.10 & $\begin{array}{c}1.57 \\
(0.95,2.62)\end{array}$ & 0.08 \\
\hline
\end{tabular}




\section{Physically Unhealthy Days \\ Mentally Unhealthy Days \\ Inactive Days due to $0-15$ vs. $16-30$ $0-15$ vs. $16-30$ Physical/Mental Health $0-15$ vs. $16-30$}

\begin{tabular}{|c|c|c|c|c|c|c|}
\hline & $\begin{array}{c}\text { Odds Ratio } \\
(95 \% \mathrm{Cl})\end{array}$ & P-Value & $\begin{array}{c}\text { Odds Ratio } \\
(95 \% \mathrm{Cl})\end{array}$ & P-Value & $\begin{array}{c}\text { Odds Ratio } \\
(95 \% \mathrm{Cl})\end{array}$ & P-Value \\
\hline $\begin{array}{l}\text { Education } \\
<9^{\text {th }} \text { Grade }\end{array}$ & Reference & & & & & \\
\hline $9^{\text {th }}-11^{\text {th }}$ Grade & $\begin{array}{c}0.62 \\
(0.35,1.08)\end{array}$ & 0.09 & $\begin{array}{c}0.66 \\
(0.39,1.14)\end{array}$ & 0.13 & $\begin{array}{c}0.63 \\
(0.22,1.82)\end{array}$ & 0.39 \\
\hline $\begin{array}{l}\text { High School } \\
\text { Graduate }\end{array}$ & $\begin{array}{c}1.68 \\
(1.00,2.92)\end{array}$ & 0.07 & $\begin{array}{c}0.61 \\
(0.34,1.11)\end{array}$ & 0.11 & $\begin{array}{c}0.66 \\
(0.27,1.61)\end{array}$ & 0.36 \\
\hline $\begin{array}{l}\text { Some College/AA } \\
\text { Degree }\end{array}$ & $\begin{array}{c}0.96 \\
(0.52,1.78)\end{array}$ & 0.90 & $\begin{array}{c}1.10 \\
(0.67,1.80)\end{array}$ & 0.70 & $\begin{array}{c}0.42 \\
(0.19,0.96)\end{array}$ & 0.04 \\
\hline $\begin{array}{l}\text { College Graduate } \\
\text { or Above }\end{array}$ & $\begin{array}{c}1.62 \\
(1.01,2.61)\end{array}$ & 0.05 & $\begin{array}{c}0.99 \\
(0.46,2.15)\end{array}$ & 0.98 & $\begin{array}{c}0.61 \\
(0.22,1.66)\end{array}$ & 0.33 \\
\hline $\begin{array}{l}\text { Poverty } \\
\quad>130 \%\end{array}$ & Reference & & & & & \\
\hline$<=130 \%$ & $\begin{array}{c}1.02 \\
(0.60,1.74)\end{array}$ & 0.94 & $\begin{array}{c}1.06 \\
(0.69,1.61)\end{array}$ & 0.80 & $\begin{array}{c}0.53 \\
(0.34,0.81)\end{array}$ & 0.0034 \\
\hline
\end{tabular}




$\begin{array}{ccc}\text { Physically Unhealthy Days } & \text { Mentally Unhealthy Days } & \text { Inactive Days due to } \\ 0-15 \text { vs. } 16-30 & 0-15 \text { vs. } 16-30 & \text { Physical/Mental Health } \\ & & 0-15 \text { vs. } 16-30\end{array}$

\begin{tabular}{|c|c|c|c|c|c|c|}
\hline & $\begin{array}{l}\text { Odds Ratio } \\
\text { (95\% Cl) }\end{array}$ & P-Value & $\begin{array}{c}\text { Odds Ratio } \\
\text { (95\% } \mathrm{Cl})\end{array}$ & P-Value & $\begin{array}{c}\text { Odds Ratio } \\
(95 \% \mathrm{Cl})\end{array}$ & P-Value \\
\hline \multicolumn{7}{|l|}{ Race/ Ethnicity } \\
\hline $\begin{array}{l}\text { Non-Hispanic } \\
\text { White }\end{array}$ & Reference & & & & & \\
\hline $\begin{array}{l}\text { Mexican- } \\
\text { American }\end{array}$ & $\begin{array}{c}0.69 \\
(0.34,1.40)\end{array}$ & 0.30 & $\begin{array}{c}1.07 \\
(0.44,2.60)\end{array}$ & 0.87 & $\begin{array}{c}0.99 \\
(0.37,2.62)\end{array}$ & 0.98 \\
\hline $\begin{array}{l}\text { Non-Hispanic } \\
\text { Black }\end{array}$ & $\begin{array}{c}1.11 \\
(0.63,1.95)\end{array}$ & 0.71 & $\begin{array}{c}0.83 \\
(0.56,1.22)\end{array}$ & 0.33 & $\begin{array}{c}0.97 \\
(0.53,1.80)\end{array}$ & 0.93 \\
\hline Other Hispanic & $\begin{array}{c}0.97 \\
(0.53,1.74)\end{array}$ & 0.90 & $\begin{array}{c}0.81 \\
(0.29,2.27)\end{array}$ & 0.69 & $\begin{array}{c}1.72 \\
(0.59,5.00)\end{array}$ & 0.32 \\
\hline $\begin{array}{l}\text { Other/ } \\
\text { Multicultural }\end{array}$ & $\begin{array}{c}1.70 \\
(0.68,4.29)\end{array}$ & 0.26 & $\begin{array}{c}1.13 \\
(0.30,4.26)\end{array}$ & 0.86 & $\begin{array}{c}1.84 \\
(0.35,8.71)\end{array}$ & 0.47 \\
\hline $\begin{array}{l}\text { Live Alone } \\
\quad \text { No }\end{array}$ & \multicolumn{4}{|c|}{ Live Alone } & & \\
\hline Yes & $\begin{array}{c}1.11 \\
(0.63,1.96)\end{array}$ & 0.73 & $\begin{array}{c}0.56 \\
(0.30,1.05)\end{array}$ & 0.07 & $\begin{array}{c}0.66 \\
(0.37,1.19) \\
\end{array}$ & 0.17 \\
\hline
\end{tabular}

${ }^{a}$ Adjusted odds ratios are odds ratios when all variables are included in the model

${ }^{b}$ Data from NHANES 2009-2012 Demographics and Questionnaire Data Files; all data are weighted in order to represent the U.S.

population. Weighting also accounts for the probability of being selected for the sample, survey nonresponse, and differences between the sample and total U.S. population

${ }^{\mathrm{C}}$ Physically unhealthy days, mentally unhealthy days, and inactive days outcomes are modeling a low number of unhealthy days $(0-15$

days vs. $16-30$ in the past 30 days)

${ }^{\mathrm{d}} \mathrm{HEI}$ (Healthy Eating index) individual total average scores over day 1 and day 2 of NHANES dietary data 
Those who had difficulty with meal prep had $76 \%$ lower odds of fewer mentally unhealthy days, compared to those who had no difficulty with meal prep $(p<0.0001)$. Compared to those who were not bothered at all by poor appetite/overeating, those who were bothered by poor appetite/overeating nearly every day had $88 \%$ lower odds of fewer mentally unhealthy days $(p<0.0001)$.

For the Inactive Days Model, the significant predictors were difficulty with meal prep, bothered by poor appetite/overeating, education, and poverty. Compared to those who had no difficulty with meal prep, those who had difficulty with meal prep had $83 \%$ lower odds of less inactive days $(p<0.0001)$. Those who were bothered by poor appetite/overeating nearly every day had $89 \%$ lower odds of less inactive days compared to those who were not all bothered by poor appetite/overeating $(p<0.0001)$. Older adults who had some college/AA degree had $58 \%$ lower odds of less inactive days compared to those with less than $9^{\text {th }}$ grade education $(p=0.04)$. Lastly, those in poverty had $47 \%$ lower odds of having less inactive days compared to those not in poverty $(p=0.0034)$ 


\section{Chapter 5: Discussion, Conclusions, and Future Research}

The main objective of this research was to examine the relationship between dietary factors (especially diet quality) and health-related quality of life in communitydwelling older adults aged 60 and above, taking into account other relevant nutritionrelated factors and demographic variables. This chapter examines the context and implications of the findings, as well as strengths/limitations of the work and directions for future research.

\subsection{Discussion}

The NHANES online dataset survey cycles from 2009-2010 and 2011-2012 were used to determine the association between diet and quality of life, specifically if diet quality was predictive of $\mathrm{HRQOL}$, in a nationally representative, non-institutionalized older adult population. Consistent with the literature (Germain et al., 2013; Gopinath et al., 2014), this thesis research found that better diet quality was associated with better HRQOL, after adjusting for key covariates. Diet quality remained a significant predictor in the HEI Continuous Model, HEI Categorical Model, and the Self-Perceived Diet Quality Model with global HRQOL as the outcome measure. This study was the first to examine the diet quality-HRQOL relationship in a nationally representative sample of US older adults.

When relevant nutrition-related and other predictor covariates were combined into one model predicting good or better HRQOL, diet quality still remained a significant predictor, supporting the original hypothesis. Those in the highest HEI quartile had 2.4 times greater odds of good or better HRQOL compared to those in the first HEI quartile. Those who self-rated their diet quality as excellent, very good, or good were 3.1 times more likely to have a good or better (vs fair/poor) HRQOL, after adjusting for demographic and nutrition-related variables. Assaf et al. (2016), Germain et al. (2013), and Rasheed and Woods (2013) also investigated the relationship between diet and 
HRQOL though in different populations and with different diet quality and outcome measures. Rasheed and Woods (2013) conducted a systematic review and metaanalysis of studies examining nutrition and quality of life in older adults in both intervention and observational studies. One of the reviewed studies, the paper by Smoliner et al. (2009), was similar to this thesis research in its cross-sectional design but not in its sample population or measurement tools. This study looked at 114 residents of a German nursing home, and the average age was 84 years old. Nutritional status was assessed using the Mini Nutritional Assessment (MNA) and HRQOL was assessed using the SF-36 (Smoliner et al., 2009). All intervention studies included in the metaanalysis were conducted in high risk, institutionalized older adult populations with nutritional supplements as the intervention (Rasheed \& Woods, 2013), and were therefore not comparable to this thesis research except for the general finding that better nutrition was related to better HRQOL. Assaf and colleagues (2016) evaluated the effect of the Women's Health Initiative Dietary Modification (WHI DM) intervention on HRQOL in 48,835 women between the ages of 50 and 79 years. The intervention goal was to reduce fat intake to $20 \%$ of total caloric needs based on the women's height, increase fruit and vegetable intake to 5 or more servings per day, and increase grains to 6 or more servings per day. To enhance adherence to the DM intervention, 18 group sessions were held throughout the year by trained nutritionists. Subjects in the DM group were successful at reducing fat intake and increasing fruit, vegetable, and grain intake, though not to the goal levels. The SF-36 was used to measure HRQOL. After one year, the SF-36 subcomponents general health, physical functioning, and vitality scores were significantly improved with the DM intervention. Germain and colleagues (2013) examined diet quality and HRQOL in one large French cohort $(n=3,005)$ of middle-aged adults. They used the Programme National Nutrition Sante (PNNS) guideline score, which is a French scoring system to measure diet quality and compliance with dietary 
guidelines, and the SF-36 to measure HRQOL. Despite the fact that Germain and others (2013) looked at diet quality and HRQOL in a French cohort and followed them over time, this study had similar predictor and outcome variables as this thesis research. However, the covariates were slightly different. Germain and colleagues (2013) looked at body mass index (BMI), employment status, smoking status, total calories, blood glucose levels, triglyceride levels, and high blood pressure in addition to sex, age, living alone, and education that this thesis research also took into account. Assaf and colleagues (2016), on the other hand, looked at HRQOL subcomponent scores by BMI, age, physical activity, and dietary total fat in their randomized controlled DM trial. Smoliner et al. (2009) looked at weight, depression, and functional status in addition to nutritional status. Despite these differences, these research groups and this thesis research arrived at the same conclusion: that better nutrition or diet quality is associated with better HRQOL.

One important finding from this research was that individual HEI values from two 24-hour recalls appeared to provide reasonable information on individual usual diet quality. Descriptive data indicated that average total $\mathrm{HEI}$ scores calculated using the $\mathrm{NCl}$ population ratio method were very similar for day one and day two of the dietary interview data. Since day one data was collected in person in the Mobile Examination Center and day two was collected through a phone interview, these findings show that the results remained consistent despite the two different collection methods that were used, and justified averaging two days of data to calculate HEI values for individuals.

An individual average HEI total score variable was calculated in order to include an actual diet quality predictor variable in regression models. To determine if two days of dietary data were reasonably reflective of usual intake, comparisons between day one and day two data were conducted. The correlation coefficient between day 1 and day 2 HEI was 0.4608 , indicating a moderate relationship between the two days. To look 
further into this relationship, quintiles for day one and day two were generated. Most older adults who were in the highest two quintiles for diet quality on day one were also in the highest two quintiles on day two. At the same time, a small percentage of older adults in the highest quintile on day one were in the lowest and next to lowest quintiles on day two. These results showed consistency of HEI scores for an individual; those with better diet quality one day tended to have better diet quality on the second day, suggesting that the $\mathrm{HEl}$ individual scores from the recalls were reasonable measures of typical diet quality.

Self-perceived diet quality was included as a predictor variable for this thesis to determine its association with self-reported overall HRQOL. Before inclusion in the logistic regression model, analyses were conducted to determine its potential relationship with the $\mathrm{NCl}$ population ratio method $\mathrm{HEI}$ total scores and the average $\mathrm{HEI}$ scores over two days variables. For both HEI variables, the highest average HEI score was in the "excellent" self-perceived diet quality response category, and scores were lower as self-perceived diet quality categories were lower, indicating an association between the two measures of diet quality. The self-perceived diet quality variable is reflective of usual intake because it asks the question, "In general, how healthy is your overall diet?" The fact that self-perceived diet quality was aligned with HEI (actual) diet quality gives further assurance that the individual HEls reasonably reflected typical diet quality.

In addition to the HEI diet quality predictor variable, other diet-related predictor variables were included in the regression models based on relevance to the older adult population. Of the 3,692 older adults that were included in this research, very few stated that they had government or community meals delivered within the last year. Interestingly, older adults who stated they had government meals delivered were less likely to have good or better HRQOL. These results were surprising because 
government meals programs are designed to improve nutritional status, as stated by Bernstein and Munoz (2012). Additionally, Frongillo and Wolfe (2010) found that older adults who received home-delivered meals had a higher intake of fruits and vegetables, indicating a higher quality diet. However, this thesis looked at meal program use controlling for diet quality, suggesting no separate benefit of meal delivery. In addition, it could be that older adults enrolled in government meal programs already had poor HRQOL to begin with, and the cross-sectional research does not allow cause and effect conclusions since these data were collected at one point in time. In fact, Wellman, Rosenzweig, and Lloyd (2002) stated that only $41 \%$ of older adults enrolled in the Elderly Nutrition Program were unable to prepare their own meals, indicating functional impairment and possible poor quality of life as a result (Gopinath et al., 2014). When Koughan and Atkinson (1993) examined older adults enrolled in a supplemental food program, they discovered that $80 \%$ of older adults were at moderate to high nutritional risk (CSFP, 2014), suggesting that studying diet quality and HRQOL in this high-risk population would be of interest rather than just in NHANES where a very low percentage of older adults participate in the ENP.

After adjusting for other predictor variables, the living situation and income were not significantly associated with HRQOL in the HEI Continuous, HEI Categorical, and Healthy Days models. However, literature suggests that these two variables do have a significant impact on overall HRQOL. Hawton and others (2011), for example, conducted a multivariate regression analysis and discovered that severe social isolation (less than monthly direct contact with family, friends, or neighbors) was associated with poor HRQOL scores, after adjusting for depression, physical co-morbidity, age, gender, living alone, accommodation type (home owner/not home owner), and employment status (Hawton et al., 2011). Another group of researchers examined household wealth by quintiles, and discovered that those in the lowest wealth quintile had the highest 
percentage of poor self-rated health and the lowest mean QOL score compared to the highest wealth quintile (Arokiasmy et al., 2015). This thesis research shows that poverty and the living situation were not significantly associated with diet quality and overall HRQOL, in contrast to the literature. However, Hawton and others (2011) did not account for meal-related variables such as difficulty with meal prep, government meal program utilization, and poor appetite/overeating as well as a few demographic variables (race/ethnicity, poverty) this thesis research took into account, suggesting that the inclusion of all these covariates affected the significant relationship between the living situation, income, and HRQOL.

Descriptive data regarding nutrition-related predictor variables indicated that most older adults had no problems with meal preparation, with a lower percentage in the younger age groups. However, some older adults stated that they don't do this activity. NHANES does not specify what "don't do" refers to, but this response could mean that older adults may not do this activity because they are physically impaired to do so. Alternatively, it could indicate that someone else may be making their meals for them, such as a spouse or caregiver, but there is no follow-up question that gives more information on the reason older adults do not do this activity. This response category also makes odds ratio interpretations complicated because it is difficult to compare "don't do this activity" to the referent group "no difficulty" since "don't do" is not a level of difficulty. However, the "don't do" responses in all the models were not statistically significant predictors. Despite this, it is informative to look at frequencies in each category to determine possible associations with age. For the difficulty using fork/knife/drinking from a cup variable, most older adults in this population had no difficulty, suggesting that these participants were not functionally impaired to carry out this daily activity. But these results were from a non-institutionalized population, which tend to be healthier and less impaired compared to those that live in a senior home 
(AOA, 2013a). One of the main reasons older adults become institutionalized is because of functional impairment; therefore, it would be interesting to examine the difficulty with food-related activities in an institutionalized population and compare it to NHANES participants, as carried out in a pilot study by Cohen-Mansfield and Jensen (2009). Similarly, most older adults stated that they are not bothered at all by poor appetite or overeating, which was higher in the older adults aged $80+$. Since increasing age tends to have a poor impact on appetite (Bernstein \& Munoz, 2012), it was surprising to find the opposite result occurring in this older adult population. Again, the cross-sectional nature of the study cannot account for changes with age, but only secular differences among age groups.

Although HEI consistently predicted better HRQOL, it was not a statistically significant predictor of physically unhealthy days (0-15 vs.16-30 in the last month), mentally unhealthy days (0-15 vs.16-30 in the last month), and inactive days due to physical or mental health issues (0-15 vs.16-30 in the last month). This may be due to the abnormal distribution of the unhealthy days variables. Because most older adults responded with "0 days" and the next highest response was "30 days" with hardly any responses in between, these variables could not be used as continuous outcome variables. Rather, they were collapsed into two categories, which included a lower amount of unhealthy days (0-15) vs. a higher amount of unhealthy days (16-30). Because most older adults reported 0 unhealthy days, it may be difficult to see any impact HEI had on these outcome variables, especially with all the covariates included in the logistic regression models. Furthermore, these healthy days measures asked about the past 30 days, whereas the overall HRQOL outcome variable asked about their health in general, which gives a better view of their overall self-perceived health.

Other nutrition-related variables were associated with these healthy days measures. Specifically, those who had difficulty with meal prep had lower odds of less 
unhealthy days compared to those who had no difficulty. Similarly, the variable bothered by poor appetite or overeating was a significant predictor; those who were bothered by poor appetite or overeating had significantly lower odds of less unhealthy days compared to those who were not bothered at all. These results suggest that meal prep difficulty and poor appetite or overeating may be contributors to unhealthy days. This is consistent with the literature. In a study conducted by Baernholdt and others (2012), a multivariate regression analysis with combined physically and mentally unhealthy days as the HRQOL outcome variable indicated that Activities of Daily Living (ADLs), race/ethnicity, and depression were significantly associated with HRQOL. Meal preparation is considered a part of ADLs, and this research suggests those who have issues with meal preparation are at a greater risk of mentally and physically unhealthy days. The poor appetite/overeating predictor variable chosen for this thesis research is part of the mental health-depression screener in NHANES, which uses a 9-item Patient Health Questionnaire (PHQ) to assess clinical depression. Since this thesis was focusing on nutrition-related predictor variables, poor appetite was the variable analyzed in relation to mental health/depression and HRQOL. Barnholdt and colleagues (2012), on the other hand, used the entire PHQ for their depression predictor variable. Poor appetite or overeating remained significant in all 6 models for this thesis, suggesting that it is an important predictor of HRQOL and a possible target for future intervention studies.

\subsection{Strengths and Limitations}

There were numerous strengths to this thesis research. First of all, studying the relationship of diet quality and other nutrition-related variables to HRQOL in a nationally representative sample of community-dwelling older adults allows results to be generalized to the non-institutionalized older adult population in the US. Combining multiple years of NHANES (2009-2010 and 2011-2012) was also a strength because it 
allowed this research to look at a larger sample size of older adults. Additionally, conducting preliminary analyses on the HEI predictor variable suggested that using two days of dietary data was reasonable to use and reflective of usual intake. Furthermore, using $\mathrm{HEl}$ as a predictor variable in addition to the self-perceived diet quality predictor variable allowed this research to examine actual diet quality in relation to HRQOL, rather than solely looking at how an older adult perceived their own overall diet.

There were several limitations in this thesis research that may have impacted interpretation of the results. First and foremost, the NHANES dataset is cross-sectional, which prevents the establishment of cause and effect relationships. Since data were collected at one point in time, it is hard to determine what came first and impacted the other-diet quality or quality of life. This limits the interpretation of results, since having a better overall HRQOL may actually allow for a better diet. Despite this, diet quality remained significant with all nutrition-related and demographic covariates included in the logistic regression models. Another limitation of NHANES is that it surveys only noninstitutionalized participants across the United States. Older adults are the age group most likely to be institutionalized in a hospital or care home compared to younger populations, and this survey excludes the older adults who are less healthy and more functionally impaired, which limits the generalizability of the results towards the healthier older adult population.

Additionally, there may be variables from NHANES relevant to diet quality and quality of life that were not taken into account for this thesis research. A chronic disease variable, for example, may have been an interesting predictor variable to include in the logistic regression models, though there was no obvious variable(s) to use. Smoking may have been a "poor health" correlate to include, though current smoking status in this cross-sectional study may not have been a good chronic condition marker, and only 8.5\% of older adults currently smoke (Ahmed et al., 2015). Logistic regression analyses 
were run with a smoking variable (current smoking yes/no) in the models, and all diet quality, meal-related, and other covariates remained significantly predictive of better HRQOL. Univariate and multivariate analyses indicated that current smoking status was not significantly predictive of better HRQOL. As mentioned, a small percentage of the older adults currently smoke, which may make the current smoking variable less useful and representative of the lifetime impact in the older adult population in the US. Furthermore, $49 \%$ of the current smoking status data in older adults in NHANES were missing, and would therefore eliminate half of the sample of older adults included in the logistic regression models. For these reasons, and the lack of alteration of conclusions about diet and HRQOL, smoking status was not included in the final logistic regression models.

$\mathrm{BMI}$, another measure sometimes correlated with chronic health conditions and included as a covariate in some studies of HRQOL, is complicated to interpret as lower $\mathrm{BMI}$ in older adults may reflect unintentional weight loss and poor health, and higher BMI in older adults is often paradoxically related to lower mortality (Chau et al., 2008). An obese BMI classification in younger populations would typically be considered a marker of poor health, but in the older population a higher BMI can be protective of frailty and poor functional status. Preliminary analyses indicated that the average BMI for older adults who reported their general health as "excellent" was $26.6 \mathrm{~kg} / \mathrm{m}^{2}$, which is classified as overweight. BMI was higher as the general health responses were poorer. The average BMI in those who reported "fair" health was $30.3 \mathrm{~kg} / \mathrm{m}^{2}$, and the average BMI was $31.7 \mathrm{~kg} / \mathrm{m}^{2}$ in those who reported "poor" health, which are both classified as obese. These results indicate that there is a possible association between BMI and HRQOL. Diet quality likely impacts BMI, which could therefore mediate and explain the observed relationship between HEI and HRQOL. Further in-depth analyses on the relationships among BMI, diet, and $\mathrm{HRQOL}$ are recommended. 
As with all observational studies, there may be additional confounders that were not measured or unknown within NHANES data that may have affected the results. Also, NHANES has a limited amount of variables specifically looking at QOL, and does not include QOL and HRQOL measurement tools such as the SF-36, which is the most common comprehensive HRQOL tool used in research on that topic (Rand Corporation, 2015)

\subsection{Conclusions and Suggestions for Future Research}

Despite some limitations, this thesis research fully examined the relationship between diet quality and quality of life in community-dwelling older adults in the US. The variables in the logistic regression models were carefully chosen based on relevancy to the overall HRQOL outcome and past research discussed in the literature review.

Overall, results indicated that diet has a significant, positive relationship to health-related quality of life, after adjusting for age, meals delivered, meals eaten at a senior center, meal prep difficulty, bothered by poor appetite or overeating, gender, marital status, education, poverty, race, and living alone. Those in the highest HEI quartile had 2.4 times greater odds of better HRQOL compared to those in the lowest HEI quartile.

Future research is needed to investigate aspects of the relationship between diet and quality of life. Since the cross-sectional nature of NHANES limits the ability to look at effects of diet on HRQOL over time, research should focus on cohort or intervention studies to determine if better diet quality can actually improve HRQOL. Because NHANES only includes non-institutionalized participants, more research is needed on institutionalized older adults since they are a higher need population that may have poorer health and nutritional status. Additionally, in the NHANES sample there was only a small number of older adults who participated in the Elderly Nutrition Program; 
therefore, research should focus on these government meal programs to determine their impact on HRQOL, as there is hardly any research on this relationship (CSFP, 2014).

Also, more research is needed on the Healthy Eating Index and the methods involved with calculating and analyzing average individual HEI scores for a population, because guidance on how to properly estimate HEI scores in a model with a health outcome is still in preparation. Research should also focus on collecting dietary data for more than two days in order to have $\mathrm{HEI}$ diet quality individual scores that are more reflective of usual, long-term intake. Since NHANES only has two days of dietary data per individual in each survey cycle, research on more than two days of data was not possible for this thesis, but the significant relationship between HEI and HRQOL warrants the need for more research on diet quality. Furthermore, there is little research on using $\mathrm{HEl}$ as an actual diet quality predictor variable with a global HRQOL outcome (Assaf et al., 2016), regardless of the number of days used to measure diet. Dietary data collected in NHANES could also be used to look at components of the HEI in addition to the total HEI scores this thesis research analyzed. Looking at specific food groups, such as fruits, vegetables, and whole grains that reflect good or better diet quality and their potential impact on HRQOL might help to clarify if certain components of the HEI were more or less predictive of HRQOL.

This thesis research revealed a significant relationship between dietary factors and quality of life in community-dwelling older adults. The connection between nutrition and HRQOL supports the need for future research on this topic, with diet quality and other meal-related variables as potential targets to improve quality of life. 


\section{REFERENCES}

Administration on Aging [AOA], (2013a). A profile of older Americans, 2013. Retrieved June 18, 2014.

Administration on Aging [AOA], (2013b). Nutrition Services. Retrieved November 12, 2014.

Ahmed, J., Homa, D.M., O'Connor, E., Babb, S.D., Caraballo, R.S., Singh, T.,...King, B.A. (2015). Morbidity and mortality weekly report (MMWR): Current cigarette smoking among adults-United States, 2005-2014.

Allard, J. P. (2001). Nutritional status and the elderly: The challenge ahead. Current Opinion in Clinical Nutrition and Metabolic Care, 4(4), 292-294.

Alzheimer's Association [AA], (2014). Alzheimer's Disease Facts and Figures. Alzheimer's \& Dementia, Volume 10, Issue 2.

Amarantos, E., Martinez, A., \& Dwyer, J. (2001). Nutrition and quality of life in older adults. The Journals of Gerontology. Series A, Biological sciences and Medical Sciences, 56 Spec No 2, 54-64.

American Thoracic Society (ATS 2007). Quality of Life Resources: Sickness Impact Profile.Retrieved on December 28, 2014.

Anyanwu, U. O., Sharkey, J. R., Jackson, R. T., \& Sahyoun, N. R. (2011). Home food environment of older adults transitioning from hospital to home. Journal of Nutrition in Gerontology and Geriatrics, 30(2), 105-121.

Assaf, A.R., Beresford, S.A., Risica, P.M., Aragaki, A., Brunner, R.L., Bowen, D.J., Naughton, M., Rosal, M.C., Snetselaar, L., \& Wenger, N. (2016). Low fat dietary pattern intervention and health-related quality of life: The Women's Health Initiative randomized controlled dietary modification trial. Journal of the Academy of Nutrition and Dietetics, 116(2), 259-271.

Baernholdt, M., Yan, G., Hinton, I., Rose, K., \& Mattos, M. (2012). Quality of life in rural and urban adults 65 years and older: Findings from the National Health and Nutrition Examination survey. The Journal of Rural Health: Official Journal of the American Rural Health Association and the National Rural Health Care Association, 28(4), 339-47.

Barr, J. T., \& Schumacher, G. E. (2003a). The need for a nutrition-related quality-oflife measure. Journal of the American Dietetic Association. 103(2). 177-180.

Barr, J.T., \& Schumacher, G.E. (2003b). Using focus groups to determine what constitutes quality of life in clients receiving medical nutrition therapy: First steps in the development of a nutrition quality-of-life survey. Journal of the American Dietetic Association. 102(7). 844-851. 
Bergner, M., Bobbitt, R. A., Carter, W. B., \& Gilson, B. S. (1981). The Sickness Impact Profile: development and final revision of a health status measure. Medical care, 19(8), 787-805

Bernstein, M., \& Munoz, N. (2012). Position of the Academy of Nutrition and Dietetics: Food and nutrition for older adults: Promoting health and wellness. Journal of the Academy of Nutrition and Dietetics, 112(8), 1255-1277.

California Department of Food and Agriculture (CDFA, 2014). Senior Farmers' Market Nutrition Program. Retrieved November 12, 2014.

Carson, T. L., Hidalgo, B., Ard, J. D., \& Affuso, O. (2014). Dietary interventions and quality of life: A systematic review of the literature. Journal of Nutrition Education and Behavior, 46(2), 90-101.

Centers for Disease Control and Prevention (CDC 2009). National Center for Health Statistics (NCHS). National Health and Nutrition Examination Survey: MEC InPerson Dietary Interviewers Procedures Manual. Hyattsville, MD: U.S. Department of Health and Human Services, Centers for Disease Control and Prevention. Retrieved from http://www.cdc.gov/nchs/data/nhanes/nhanes_09_10/DietaryInterviewers_Inpers on.pdf

Centers for Disease Control and Prevention (CDC 2010). Office of Disease Prevention and Promotion (ODPHP) Healthy people 2010. Retrieved September 15, 2014.

Centers of Disease Control and Prevention (CDC 2011a). Health-Related Quality of Life (HRQOL): Concepts. Retrieved from http://www.cdc.gov/hrqol/concept.htm

Centers of Disease Control and Prevention (CDC 2011b). Health-Related Quality of Life (HRQOL): Methods and Measures. Retrieved from http://www.cdc.gov/hrqol/methods.htm

Centers for Disease Control and Prevention (CDC 2012). Health-Related Quality of Life (HRQOL): CDC HRQOL-14 "Healthy Days Measure." Retrieved from http://www.cdc.gov/hrqol/hrqol14_measure.htm.

Centers for Disease Control and Prevention (CDC 2013a). National Center for Health Statistics (NCHS). National Health and Nutrition Examination Survey: Analytic Guidelines, 2011-2012. Hyattsville, MD: U.S. Department of Health and Human Services, Centers for Disease Control and Prevention. Retrieved from http://www.cdc.gov/nchs/data/nhanes/analytic_guidelines_11_12.pdf

Centers for Disease Control and Prevention (CDC 2013b). National Center for Health Statistics (NCHS). National Health and Nutrition Examination Survey: Plan and Operations, 1999-2010. Hyattsville, MD: U.S. Department of Health and Human Services, Centers for Disease Control and Prevention. Retrieved from http://www.cdc.gov/nchs/data/series/sr_01/sr01_056.pdf 
Centers for Disease Control and Prevention (CDC 2013c). National Center for Health Statistics (NCHS). National Health and Nutrition Examination Survey: Analytic Guidelines, 2007-2010. Hyattsville, MD: U.S. Department of Health and Human Services, Centers for Disease Control and Prevention. Retrieved from http://www.cdc.gov/nchs/data/series/sr_02/sr02_161.pdf

Centers for Disease Control and Prevention (CDC 2013d). National Center for Health Statistics (NCHS). National Health and Nutrition Examination Survey: 2011-2012 Data Documentation, Codebook and Frequencies-Current Health Status. Hyattsville, MD: U.S. Department of Health and Human Services, Centers for Disease Control and Prevention. Retrieved from http://www.cdc.gov/nchs/data/nhanes/analytic_guidelines_11_12.pdf

Centers for Disease Control and Prevention (CDC 2013e). National Center for Health Statistics (NCHS). National Health and Nutrition Examination Survey: 2011-2012 Data Documentation, Codebook and Frequencies-Demographic Variables and Sample Weights. Hyattsville, MD: U.S. Department of Health and Human Services, Centers for Disease Control and Prevention. Retrieved from http://www.cdc.gov/nchs/data/series/sr_02/sr02_159.pdf

Centers for Disease Control and Prevention (CDC 2013f). National Center for Health Statistics (NCHS). National Health and Nutrition Examination Survey: Estimation Procedures, 2007-2010. Hyattsville, MD: U.S. Department of Health and Human Services, Centers for Disease Control and Prevention. Retrieved from http://www.cdc.gov/nchs/data/series/sr_02/sr02_159.pdf

Centers for Disease Control and Prevention (CDC 2013g). National Center for Health Statistics (NCHS). National Health and Nutrition Examination Survey: Continuous NHANES Web Tutorial: Preparing an Analytic Dataset. Hyattsville, MD: U.S. Department of Health and Human Services, Centers for Disease Control and Prevention. Retrieved from http://www.cdc.gov/nchs/data/series/sr_01/sr01_056.pdf

Centers for Disease Control and Prevention (CDC 2014a). National Center for Health Statistics (NCHS). National Health and Nutrition Examination Survey: Overview. Hyattsville, MD: U.S. Department of Health and Human Services, Centers for Disease Control and Prevention. Retrieved from http://www.cdc.gov/nchs/nhanes/about_nhanes.htm

Centers for Disease Control and Prevention (CDC 2014b). National Center for Health Statistics (NCHS). National Health and Nutrition Examination Survey: Dietary Interview-Individual Foods-First Day. Hyattsville, MD: U.S. Department of Health and Human Services, Centers for Disease Control and Prevention. Retrieved from http://wwwn.cdc.gov/Nchs/Nhanes/2011-2012/DR1IFF_G.htm 
Centers for Disease Control and Prevention (CDC 2015a). National Center for Health Statistics (NCHS). National Health and Nutrition Examination Survey: 2011-2012 Data Documentation, Codebook and Frequencies-Demographic Variables and Sample Weights. Hyattsville, MD: U.S. Department of Health and Human Services, Centers for Disease Control and Prevention. Retrieved from http://wwwn.cdc.gov/Nchs/Nhanes/2011-2012/DEMO_G.htm

Centers for Disease Control and Prevention (CDC 2015b). National Center for Health Statistics(NCHS). National Health and Nutrition Examination Survey: 2011-2012 Data Documentation, Codebook and Frequencies-Diet Behavior and Nutrition. Hyattsville, MD: U.S. Department of Health and Human Services, Centers for Disease Control and Prevention. Retrieved from http://wwwn.cdc.gov/Nchs/Nhanes/2011-2012/DBQ_G.htm

Centers for Disease Control and Prevention (CDC 2015c). National Center for Health Statistics (NCHS). National Health and Nutrition Examination Survey: 2011-2012 Data Documentation, Codebook and Frequencies-Mental Health-Depression Screener. Hyattsville, MD: U.S. Department of Health and Human Services, Centers for Disease Control and Prevention. Retrieved from http://wwwn.cdc.gov/Nchs/Nhanes/2011-2012/DPQ_G.htm

Centers for Disease Control and Prevention (CDC 2015d). National Center for Health Statistics (NCHS). National Health and Nutrition Examination Survey: 2011-2012 Data Documentation, Codebook and Frequencies-Physical Functioning. Hyattsville, MD: U.S. Department of Health and Human Services, Centers for Disease Control and Prevention. Retrieved from http://wwwn.cdc.gov/Nchs/Nhanes/2011-2012/PFQ_G.htm

Chau, D., Cho, L.M., Jani, P., \& St. Jeor, S.T. (2008). Individualizing recommendations for weight management in the elderly. Current Opinion in Clinical Nutrition and Metabolic Care. 11, 27-31.

Choi, N. G., Lee, A., \& Goldstein, M. (2011). Meals on Wheels: Exploring potential for and barriers to integrating depression intervention for homebound older adults. Home Health Care Services Quarterly. 30(4). 214-230.

Cohen-Mansfield, J., \& Jensen, B. (2009). A comparison of eating practices of independently living older adults in private residences and in senior retirement housing: a pilot study. Journal of Nutrition for the Elderly. 28(4), 394-407.

Commodity Supplemental Food Program [CSFP], (2014). In Effects of Food Assistance and Nutrition Programs on Nutrition and Health (pp. 293-296). Economic Research Service/USDA.

Corle, D.K., Sharbaugh, C., Mateski, D.J., Coyne, T., Paskett, E.D., Cahill, J., . . . Schatzkin, A. (2001). Self-rated quality of life measures: Effect of change to a low-fat, high-fiber, fruit and vegetable enriched diet. Annals of Behavioral Medicine, 23(3), 198-207.

Cui, W., Zack, M.M., \& Zahran, H.S. (2015). Health-related quality of life among United States adolescents. Journal of Pediatrics. 166(2), 358-364. 
Deierlein, A. L., Morland, K. B., Scanlin, K., Wong, S., \& Spark, A. (2014). Diet quality of urban older adults age 60 to 99 years: The cardiovascular health of seniors and built environment study. Journal of the Academy of Nutrition and Dietetics, 114(2), 279-187.

Drewnowski, A., \& Evans, W. J. (2001). Nutrition, physical activity, and quality of life in older adults: Summary. The Journals of Gerontology. Series A, Biological Sciences and Medical Sciences. 89-94.

Dubuc, N., Dubois, M.-F., Raîche, M., Gueye, N., \& Hébert, R. (2011). Meeting the home-care needs of disabled older persons living in the community: Does integrated services delivery make a difference? BMC Geriatrics. 11(67). 1-13.

Edington, J., Barnes, R., Bryan, F., Dupree, E., Frost, M., Hickson, J., . . Coles, S.J. (2004). A prospective randomized controlled trial of nutritional supplementation in malnourished elderly in the community: clinical and health economic outcomes. Clinical Nutrition. 23. 195-204.

Elsner, R. (2002). Changes in eating behavior during the aging process. Eating Behaviors. 15-43.

EuroQol Research Foundation (EQRF, 2015). About EQ-5D. Accessed March 1, 2015.

Federal Interagency Forum on Aging-Related Statistics. (FIFARS 2012). Older Americans 2012: Key indicators of well-being. Federal Interagency Forum on Aging-Related Statistics, Washington, DC: U.S. Government Printing Office. July 2012.

Felce, D., \& Perry, J. (1995). Quality of life: Its definition and measurement. Research in Developmental Disabilities. 16(1). 51-74.

Food and Drug Administration (FDA 2014). Using the nutrition facts label: A how-to guide for older adults. U.S Department of Health and Human Services. Retrieved September 15, 2014.

Ford, D.W., Hartman, T.J., Still, C., Wood, C., Mitchell, D.C., Bailey, R., SmiciklasWright, H., Coffman, D.L., \& Jensen, G.L. (2014). Diet quality and body mass index are associated with health care resource use in rural older adults. Journal of the Academy of Nutrition and Dietetics, 114(12),1932-1938.

Frazier-Wood, A.C., Kim, J., Davis, J.S., Jung, S.Y., \& Chang, S. (2015). In crosssectional observations, dietary quality is not associated with CVD risk in women; in men the positive association is accounted for by BMI. British Journal of Nutrition, 113, 1244-1253.

Freedman, L. S., Guenther, P.M., Krebs-Smith, S.M., Dodd, K.W., \& Midthune, D. (2010). A population's distribution of healthy eating index-2005 component scores can be estimated when more than one 24 -hour recall is available. Journal of Nutrition, 140(8), 1529-1534. 
Frongillo, E. A., Isaacman, T. D., Horan, C. M., Wethington, E., \& Pillemer, K. (2010). Adequacy of and satisfaction with delivery and use of home-delivered meals. Journal of Nutrition for the Elderly, 29(2), 211-226.

Frongillo, E. A., \& Wolfe, W. S. (2010). Impact of participation in home-delivered meals on nutrient intake, dietary patterns, and food insecurity of older persons in New York state. Journal of Nutrition for the Elderly, 29(3), 293-310.

Gallegos-Carrillo, K., García-Peña, C., Mudgal, J., Romero, X., Durán-Arenas, L., \& Salmerón, J. (2009). Role of depressive symptoms and comorbid chronic disease on health-related quality of life among community-dwelling older adults. Journal of Psychosomatic Research, 66(2), 127-135.

Gariballa, S., \& Forster, S. (2007). Dietary supplementation and quality of life of older patients: A randomized, double-blind, placebo-controlled trial. Journal of the American Geriatrics Society. 55(12). 2030-2034.

Germain, L., Latarche, C., Kesse-Guyot, E., Galan, P., Hercberg, S., \& Briançon, S. (2013). Does compliance with nutrition guidelines lead to healthy aging? A quality-of-life approach. Journal of the Academy of Nutrition and Dietetics, 113(2), 228-240.

Gollub, E. A., \& Weddle, D. O. (2004). Improvements in nutritional intake and quality of life among frail homebound older adults receiving home-delivered breakfast and lunch. Journal of the American Dietetic Association, 104(8), 1227-1235.

Gopinath, B., Russell, J., Flood, V. M., Burlutsky, G., \& Mitchell, P. (2014). Adherence to dietary guidelines positively affects quality of life and functional status of older adults. Journal of the Academy of Nutrition and Dietetics, 114(2), 220-229.

Groessl, E. J., Kaplan, R. M., Rejeski, W. J., Katula, J. A., King, A. C., Frierson, G., Glynn, N. W., et al. (2007). Health-related quality of life in older adults at risk for disability. American Journal of Preventive Medicine, 33(3), 214-218

Grunert, K. G., Dean, M., Raats, M. M., Nielsen, N. A., \& Lumbers, M. (2007). A measure of satisfaction with food-related life. Appetite, 49(2), 486-493.

Guenther, P., Casavale, K., Reedy, J., Kirkpatrick, S., Hiza, H., Kuczynski, K., Kahle, L., Krebs-Smith, S. (2013). Update of the Healthy Eating Index: HEI-2010. Journal of the Academy of Nutrition and Dietetics.

Guenther, P.M., Kirkpatrick, S.I., Reedy, J., Krebs-Smith, S.M., Buckman, D.W., Dodd, K.W.,...Carroll, R.J. (2014). The Healthy Eating Index-2010 is a valid and reliable measure of diet quality according to the 2010 Dietary Guidelines for Americans. Journal of Nutrition, 144, 399-407.

Hardy, M.A., \& Bryman, A. (2009). Handbook of Data Analysis. London, England: SAGE Publications. 
Hawton, A., Green, C., Dickens, A. P., Richards, S. H., Taylor, R. S., Edwards, R., Greaves, C. J., et al. (2011). The impact of social isolation on the health status and health-related quality of life of older people. Quality of Life Research,20(1), 57-67.

Haywood KL, Garratt AM, Schmidt LJ, Mackintosh AE, Fitzpatrick R (2004). Health Status and Quality of Life in Older People: a Structured Review of Patientreported Health Instruments. Report from the Patient reported Health Instruments Group (formerly the Patient-assessed Health Outcomes Programme) to the Department of Health, April 2004.

Haywood, K.L., Garratt, A.M., \& Fitzpatrick, R. (2004). Older people specific health status and quality of life: a structured review of self-assessed instruments. Journal of Evaluation in Clinical Practice, 11(4), 315-327.

Hickson, M., \& Frost, G. (2004). An investigation into the relationships between quality of life, nutritional status and physical function. Clinical Nutrition. 23, 213-221.

Humbert, I., \& Robbins, J. (2008). Dysphagia in the elderly. Physical Medicine Clinics of North America. 19(4), 853-866.

Johansson, Y., Bacjrach-Lindstrom, M., Cartensen, J., \& Ek, A. (2009). Malnutrition in a home-living older population: prevalence, incidence and risk factors. A prospective study. Journal of Clinical Nursing. 18, 1354-1364.

Kamp, B. (2010). Position of the American Dietetic Association, American Society for Nutrition, and Society for Nutrition Education: Food and nutrition programs for community-residing older adults. Journal of the American Dietetic Association.

Kirkman, M. S., Briscoe, V. J., Clark, N., Florez, H., Haas, L. B., Halter, J. B., Huang, E. S., et al. (2012). Diabetes in older adults. Diabetes Care. 35 (12). 2650-2664.

Koughan, N., \& Atkinson, C. (1993). Nutrition Screening Initiative and the Louisiana Food for Seniors experience. Journal of the Louisiana State Medical Society. 145(10), 447-449.

Kuczmarski, M., \& Weddle, D. (2005) Position paper of the American Dietetic Association: Nutrition across the spectrum of aging. Journal of the American Dietetic Association, 105(4), 616-633.

Kunkel, M. E., Luccia, B., \& Moore, A. C. (2003). Evaluation of the South Carolina Seniors Farmers' Market Nutrition Education Program. Journal of the American Dietetic Association, 103(7), 880-883.

Lee, J. S., \& Frongillo, E. A. (2001). Nutritional and health consequences are associated with food insecurity among U.S. elderly persons. The Journal of nutrition, 131(5), 1503-1509.

Lee, J. S., Sinnett, S., Bengle, R., Johnson, M. A., \& Brown, A. (2011). Unmet Needs for the Older Americans Act Nutrition Program. Journal of Applied Gerontology, 30(5), 587-606. 
Mahadevan, M., Hartwell, H. J., Feldman, C. H., Ruzsilla, J. A., \& Raines, E. R. (2014). Assisted-living elderly and the mealtime experience. Journal of Human Nutrition and Dietetics, 27(2), 152-161. Blackwell Publishing Ltd.

Meneely, L., Strugnell, C., \& Burns, A. (2009). Elderly consumers and their food store experiences. Journal of Retailing and Consumer Services, 16(6), 458-465.

Millen, B. E., Ohls, J. C., Ponza, M., \& McCool, A. C. (2002). The Elderly Nutrition Program. Journal of the American Dietetic Association. 102(2). 234-240.

National Alliance for Caregiving (NAC, 2009). Caregiving in the US: 2009. Retrieved October 26, 2014.

National Cancer Institute (NCI 2015). National Institutes of Health (NIH). Epidemiology And Genomics Research: The Healthy Eating Index-2010. Rockville, MD. Retrieved from http://epi.grants.cancer.gov/about/contact.html

National Commodity Supplemental Food Programs Association (NCSFPA, 2013). 20122013 Senior Snap Survey. Retrieved November 12, 2014.

Nguyen, B.T., Shuval, K., Bertmann, F., \& Yaroch, A.L. (2015). The supplemental nutrition assistance program, food insecurity, dietary quality, and obesity among US adults. American Journal of Public Health 105(7), 1453-1459.

Office of Disease Prevention and Health Promotion (ODPHP, 2014a). Health-Related Quality of Life and Well-Being. Retrieved on December 29, 2014.

Office of Disease Prevention and Health Promotion (ODPHP 2014b). Healthy People 2020 Topics \& Objectives: Older Adults. Washington, DC: U.S. Department of Health and Human Services. Retrieved from http://www.healthypeople.gov/2020/topics-objectives/topic/older-adults

Office of Disease Prevention and Health Promotion (ODPHP 2015). Healthy People 2020: About Healthy People. Washington, DC: U.S. Department of Health and Human Services. Retrieved from http://www.healthypeople.gov/2020/AboutHealthy-People

Park, Y.-H., Song, M., Cho, B.-L., Lim, J.-Y., Song, W., \& Kim, S.-H. (2010). The effects of an integrated health education and exercise program in community-dwelling older adults with hypertension: A randomized controlled trial. Patient education and counseling, 82(1), 133-137.

Payette, H., Boutier, V., Coulombe, C., \& Gray-Donald, K. (2002). Benefits of nutritional supplementation in free-living, frail, undernourished elderly people: A prospective randomized community trial. Journal of the American Dietetic Association. 102(8). 1088-1095.

Payette, H., \& Shatenstein, B. (2005). Determinants of healthy eating in communitydwelling elderly people. Canadian Journal of Public Health. 96(3). 27-35. 
Raad, J. (2014). Rehab Measures: Short Form 12 item (version 2) Health Survey. Rehabilitation Measures Database. Retrieved on December 23, 2014.

Rand Corporation (2015). 36-Item Short Form Survey from the RAND Medical Outcomes Study. Retrieved February 14, 2015.

Rasheed, S., \& Woods, R. T. (2013). Malnutrition and quality of life in older people: A systematic review and meta-analysis. Ageing Research Review. 12(2). 561-566.

Rehabilitation Measures Database (RMB 2010). Rehab Measures: Sickness Impact Profile. Retrieved on December 28, 2014.

SAS Institute Inc. (2015). Cary, NC. Retrieved from http://www.sas.com/en_us/contact.html

Schünemann, H. J., Sperati, F., Barba, M., Santesso, N., Melegari, C., Akl, E. A., Guyatt, G., et al. (2010). An instrument to assess quality of life in relation to nutrition: item generation, item reduction and initial validation. Health and quality of life outcomes, 8, 26.

Smoliner, C., Norman, K., Wagner, K.H., Hartig, W., Lochs, H., Pirlich, M. (2009). Malnutrition and depression in the institutionalized elderly. British Journal of Nutrition. 102 (2009), 1663-1667.

Soni, A. \& Roemer, M. (2011). Top five most costly conditions among the elderly, age 65 and older, 2008: Estimates for the U.S. Civilian noninstitutionalized adult population. Medical Expenditure Panel Survey. 1-5.

Tanner, E.K., Martinez, I.L., \& Harris, M. (2014). Examining functional and social determinants of depression in community-dwelling older adults: implications for practice. Geriatric Nursing. 35(3), 236-240.

Thomas, L., Ghiselli, R., \& Almanza, B. (2011). Congregate meal sites participants: Can they manage their diets? International Journal of Hospitality Management, 30(1), $31-37$.

United States Department of Agriculture (USDA, 2013). Measuring the effect of supplemental nutrition assistance program (SNAP) participation on food security. Retrieved November 12, 2014.

United States Department of Agriculture Agricultural Research Service (USDA-ARS, 2014). Food Patterns Equivalents Database. Retrieved from http://www.ars.usda.gov/Services/docs.htm?docid=23871

United States Department of Agriculture Center for Nutrition Policy and Promotion (USDACNPP, 2013). Healthy Eating Index-Fact Sheet no.2. Retrieved from http://www.cnpp.usda.gov/sites/default/files/healthy_eating_index/CNPPFactShe etNo2.pdf 
United States Department of Agriculture Center for Nutrition Policy and Promotion (USDACNPP, 2014). Healthy Eating Index. Retrieved from

http://www.cnpp.usda.gov/HealthyEatingIndex

United States Department of Agriculture Food and Nutrition Service (USDAFNS, 2014). Supplemental Nutrition Assistance Program (SNAP). Retrieved November 12, 2014.

United States Department of Agriculture Food and Nutrition Service (USDAFNS, 2014a). Characteristics of supplemental assistance program households: Fiscal year 2012. Retrieved November 12, 2014.

United States Department of Agriculture Food and Nutrition Service (USDAFNS, 2014b). Commodity Supplemental Food Program (CSFP). Retrieved November 12, 2014.

United States Department of Agriculture Food and Nutrition Service (USDAFNS, 2014c). Senior Farmers' Market Nutrition Program (SFMNP). Retrieved November 12, 2014.

United States Department of Health and Human Services (USDHHS, 2014). Healthy People 2020: Topics and objectives. Retrieved November 26, 2014.

United States Department of Health and Human Services (USDHHS, 2015). Administration for Community Living, Administration on Aging (AOA), Aging Statistics. Washington DC, 20201. Retrieved from http://www.aoa.acl.gov/Aging_Statistics/index.aspx

University of Washington, 2011. World Health Organization Quality of Life Instruments (WHOQOL-BREF). Retrieved on December 23, 2014.

Vesnaver, E., \& Keller, H. H. (2011). Social Influences and Eating Behavior in Later Life: A Review. Journal of Nutrition in Gerontology and Geriatrics. 30(1). 2-23.

Vespa, B., Lewis, J., \& Kreider, R. (2013). America's Families and Living Arrangements: 2012 Population characteristics. United States Census Bureau. $1-$ 34.

Ware, J. (2000). SF-36 Health Survey Update. Retrieved from SF-36.org.

Wellman, N. S., Rosenzweig, L. Y., \& Lloyd, J. L. (2002). Thirty years of the Older Americans Nutrition Program. Journal of the American Dietetic Association. 102(3). 348-350.

World Health Organization (WHO, 2003). WHO definition of Health. Retrieved on December 1, 2014.

World Health Organization (WHO, 2014). WHO Quality of Life-Bref (WHOQOLBREF). Retrieved August 2, 2014. 
Wunderlich, S., Brusca, J., Johnson-Austin, M., Bai, Y., \& O' Malley, M. (2012). Eating behaviors of older adults participating in government-sponsored programs with different demographic backgrounds. Global Journal of Health Science. 4(6) 204215.

Ziliak, J.P., \& Gundersen, C. (2011). Food Insecurity Among Older Adults: A report submitted to AARP foundation. 


\section{APPENDICES}

\section{Appendix A \\ Short Form-36 Survey Instrument for Measuring Quality of Life}

1. In general, would you say your health is:

$\begin{array}{ll}\text { Excellent } & 1 \\ \text { Very good } & 2 \\ \text { Good } & 3 \\ \text { Fair } & 4 \\ \text { Poor } & 5\end{array}$

2. Compared to one year ago, how would you rate your health in general now? Much better now than one year ago 1 Somewhat better than one year ago 2 About the same Somewhat worse than one year ago 4 Much worse than one year ago 5

The following items are about activities you might do during a typical day. Does your health now limit you in these activities? If so, how much?

$$
\begin{gathered}
\text { Yes, limited } \\
\text { a lot }
\end{gathered}
$$

$$
\begin{gathered}
\text { Yes, limited } \\
\text { a little }
\end{gathered}
$$

No, not limited at all

3. Vigorous activities, such as running, lifting heavy objects, participating in strenuous sports

4. Moderate activities, such as moving a table, pushing a vacuum cleaner, bowling, or playing golf

5. Lifting or carrying groceries

6. Climbing several flights of stairs

7. Climbing one flight of stairs

8. Bending, kneeling, or stopping

9. Walking more than a mile

10. Walking several blocks

11. Walking one block

12. Bathing or dressing yourself

During the past 4 weeks, have you had any of the following problems with your work or other regular daily activities as a result of your physical health?

$\begin{array}{cc}\text { Yes } & \text { No } \\ 1 & 2\end{array}$

13. Cut down the amount of time you spent on work or other activities

14. Accomplished less than you would like 
15. Were limited in the kind of work or other activities

16. Had difficulty performing the work or other activities

During the past 4 weeks, have you had any of the following problems with your work or other regular daily activities as a result of any emotional problems (such as feeling depressed or anxious)?

$$
\text { Yes No }
$$

\section{2}

17. Cut down the amount of time you spent on work or other activities

18. Accomplished less than you would like

19. Didn't do work or other activities as carefully as usual

20. During the past 4 weeks, to what extent has your physical health or emotional problems interfered with your normal social activities with family, friends, or neighbors, or groups?

$\begin{array}{ll}\text { Not at all } & 1 \\ \text { Slightly } & 2 \\ \text { Moderately } & 3 \\ \text { Quite a bit } & 4 \\ \text { Extremely } & 5\end{array}$

21. How much bodily pain have you had during the past 4 weeks?

$\begin{array}{ll}\text { None } & 1 \\ \text { Very mild } & 2 \\ \text { Mild } & 3 \\ \text { Moderate } & 4 \\ \text { Severe } & 5 \\ \text { Very severe } & 6\end{array}$

22. During the past 4 weeks, how much did pain interfere with your normal work (including both work outside the home and housework?

$\begin{array}{ll}\text { Not at all } & 1 \\ \text { A little bit } & 2 \\ \text { Moderately } & 3 \\ \text { Quite a bit } & 4 \\ \text { Extremely } & 5\end{array}$

These questions are about how you feel and how things have been with you during the past 4 weeks. For each question, please give one answer that comes closest to the way you have been feeling. How much of the time during the past 4 weeks...

$\begin{array}{llllll}\begin{array}{l}\text { All of } \\ \text { the }\end{array} & \begin{array}{l}\text { Most of } \\ \text { the } \\ \text { time }\end{array} & \begin{array}{l}\text { A good bit } \\ \text { of the } \\ \text { time }\end{array} & \begin{array}{l}\text { Some of } \\ \text { the } \\ \text { time }\end{array} & \begin{array}{l}\text { A little } \\ \text { bit of } \\ \text { the time }\end{array} & \begin{array}{l}\text { None of } \\ \text { the } \\ \text { time }\end{array} \\ \text { [1] } & {[2]} & {[3]} & {[4]} & {[5]} & {[6]}\end{array}$

23. Did you feel full of pep?

24. Have you been a very Nervous person?

25. Have you felt so down 
Down in the dumps that

Nothing could cheer you up?

26. Have you felt calm and

Peaceful?

27 . Did you have a lot of energy?

28. Have you felt downhearted

And blue?

29. Did you feel worn out?

30. Have you been a happy

Person?

31. Did you feel tired?

32. During the past 4 weeks, how much of the time has your physical health or emotional problems interfered with your social activities (like visiting friends, relatives, etc.)?

$\begin{array}{ll}\text { All of the time } & 1 \\ \text { Most of the time } & 2 \\ \text { Some of the time } & 3 \\ \text { A little of the time } & 4 \\ \text { None of the time } & 5\end{array}$

How TRUE or FALSE is each of the following statements for you.

$\begin{array}{ccccc}\text { Definitely } & \text { Mostly } & \text { Don't } & \text { Mostly } & \text { Definitely } \\ \text { True } & \text { True } & \text { Know } & \text { False } & \text { False } \\ 1 & 2 & 3 & 4 & 5\end{array}$

33. I seem to get sick a little easier than other people

34. I am as healthy as anybody I know

35. I expect my health to get worse

36. My health is excellent 


\section{Appendix B}

Short-Form 36 Scales and Associated Questions

\begin{tabular}{|l|c|}
\hline \multicolumn{1}{|c|}{ Scales } & Question Numbers \\
\hline Physical Functioning (PF) & $3-12$ \\
\hline Role-Physical (RP) & $13-16$ \\
\hline Bodily Pain (BP) & $21-22$ \\
\hline General Health (GH) & $1-2,33-36$ \\
\hline Vitality (VT) & $23,27,29,31$ \\
\hline Social Functioning (SF) & 20,32 \\
\hline Role-Emotional (RE) & $17-19$ \\
\hline Mental Health (MH) & $24,25,26,28,30$ \\
\hline
\end{tabular}

Data from Ware (2000). 


\section{Appendix C}

CDC's Healthy Days Questionnaire for Measuring Health-Related Quality of Life

1. Would you say that in general your health is:

$\begin{array}{ll}\text { a. Excellent } & 1 \\ \text { b. Very Good } & 2 \\ \text { c. Good } & 3 \\ \text { d. Fair } & 4 \\ \text { e. Poor } & 5 \\ \text { Don't know/Not sure } & 7 \\ \text { Refused } & 9\end{array}$

2. Now thinking about your physical health, which includes physical illness and injury, for how many days during the past 30 days was your physical health not good?
a. Number of Days
b. None
$\overline{8} \overline{8}$
Don't know/Not sure
77
Refused
99

3. Now thinking about your mental health, which includes stress, depression, and problems with emotions, for how many days during the past 30 days was your mental health not good?
a. Number of Days
b. None
$\overline{8} \overline{8}$
Don't know/Not sure
77
Refused
99

4. During the past 30 days, for about how many days did poor physical or mental health keep you from doing your usual activities, such as self-care, work, or recreation?

a. Number of Days

b. None

$\overline{8} \overline{8}$

Don't know/Not sure $\quad 77$

Refused

99

From CDC (2012). 


\section{Appendix D}

CDC's Activity Limitations Questionnaire for Measuring Health-Related Quality of Life

1. Are you LIMITED in any way in any activities because of any impairment or health problem?
a. Yes
1
b. No
2
Don't know/not sure
7
Refused
9

2. What is the MAJOR impairment or health problem that limits your activities?
a. Arthritis/rheumatism
01
b. Back of neck problem
02
c. Fractures, bone/joint injury
03
d. Walking problem
04
e. Lung/breathing problem
05
f. Hearing problem
06
g. Eye/vision problem
07
h. Heart problem
08
i. Stroke problem
09
j. Hypertension/high blood pressure $\quad 10$
k. Diabetes 11
I. Cancer 12
m. Depression/anxiety/emotional problem $\quad 13$
n. Other impairment/problem 14
Don't know/Not sure $\quad 7$
Refused 9

3. For HOW LONG have your activities been limited because of your major impairment or health problem?
a. Days
b. Weeks
c. Months
d. Years

Don't know/Not sure

Refused

$1--$
$2--$
$3--$
$4--$
777
999

4. Because of any impairment or health problem, do you need the help of other persons with your PERSONAL CARE needs, such as eating, bathing, dressing, or getting around the house?
a. Yes
1
b. No
2
Don't know/Not sure
7
Refused
9

5. Because of any impairment or health problem, do you need the help of other persons in handling your ROUTINE needs, such as everyday household chores, doing necessary business, shopping, or getting around for other purposes?
a. Yes
1
b. No
2
Don't know/Not sure
Refused

From CDC (2012). 


\section{Appendix E}

CDC's Healthy Days Symptoms Questionnaire for Measuring Health-Related Quality of Life

1. During the past 30 days, for about how many days did PAIN make it hard for you to do your usual activities, such as self-care, work, or recreation?
a. Number of Days
b. None
$\overline{8} \overline{8}$
Don't know/Not sure
77
Refused
99

2. During the past 30 days, for about how many days have you felt SAD, BLUE, or DEPRESSED?
a. Number of Days
b. None
$\overline{8} \overline{8}$
Don't know/Not sure
77
Refused
99

3. During the past 30 days, for about how many days have you felt WORRIED, TENSE, or ANXIOUS?
a. Number of Days
b. None
$\overline{8} \overline{8}$
Don't know/Not sure
77
Refused
99

4. During the past 30 days, for about how many days have you felt you did NOT get ENOUGH REST or SLEEP?

a. Number of Days

b. None

$\overline{8} \overline{8}$

Don't know/Not sure $\quad 77$

Refused

99

5. During the past 30 days, for about how many days have you felt VERY HEALTHY AND FULL OF ENERGY?

a. Number of Days

b. None

$\overline{8} \overline{8}$

Don't know/Not sure

Refused

77

99

From CDC (2012). 


\section{Appendix F \\ Nutrition Quality of Life Survey Version 1.4}

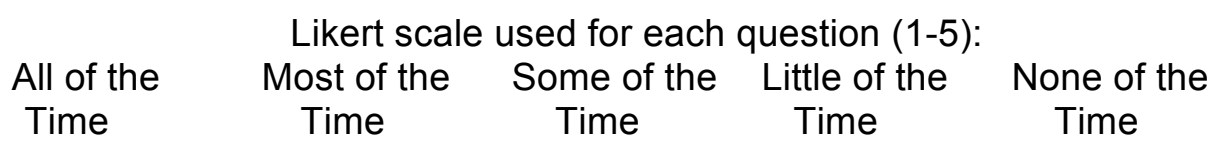

During the last 2 weeks:

1. I ate enough food to be satisfied

2. I had plenty of choice in the food I ate

3. I was hungry between meals

4. Food was on my mind

5. I sneaked food

6. I tasted and enjoyed food without guilt

7. I could afford to buy the food that was best for me

8. I took time to eat the food that was best for me

9. I, or someone else, took time to shop and prepare the food that was best for me

During the last 2 weeks:

10. Liked the way I look

11. Liked the way my clothes fit

12. Beat myself up when I ate the food I shouldn't have

13. Took time for myself

14. Was pleased with the way I managed what I ate

15. Was confused about the food I should eat

16. Rewarded myself with food

17. Was happy with the food I ate

18. Felt guilty about the food I ate

19. Felt that food was controlling me

20. Felt depressed about the way I look

21. Felt depressed about the food I ate

22. Felt that changing the food I ate would make life more enjoyable for me

23. Was frustrated about limiting the food I ate

24. Was frustrated about how long it too to improve my food-related condition

25. Was angry that I had to change what and how I ate

During the last 2 weeks:

26. My family/friends have nagged me about the food I ate

27. My food needs have created stress with my family/friends

28. I had problems going out to eat with my family/friends

29. I have cut down the amount of time I spend on work or other activities because of my food-related condition

30. I had someone I could talk to who understood the struggles I have had with food

31. My family/friends made it difficult to stick to the food I thought I should eat

32. My food-related condition has caused problems with sexual relations

During the last 2 weeks my food-related condition has given me trouble in:

33. Walking at a moderate pace for 30 minutes

34. Walking slowly for 10 minutes

35. Walking up a flight of stairs

36. Bending or kneeling to pick things up

37. Getting up off the floor 
38. Needing to use the bathroom so often I couldn't go out of the house

39. Getting a good night's sleep

40. Breathing comfortably

41. Having enough energy to do what I wanted to do

During the last 2 weeks I:

42. Knew what type of food I should have been eating for my healthy lifestyle

43. Knew the amount of food I could eat

44. Knew when to eat

45. Made healthy food choices

46. Ate the recommended amount of food

47. Was eating when I should be eating

48. Planned ahead to have healthy food when I needed it

49. I felt confident that I could trust myself when faced with difficult food choices 50. I felt confident that I would be able to live the rest of my life with these changes in my food

From MNT Profile NQoL (2002). 


\section{Appendix G}

Food Patterns Components in the Food Patterns Equivalents Database

1. Total fruit

2. Citrus, melons, and berries

3. Other fruits

4. Fruit juice

5. Total vegetables

6. Dark green vegetables

7. Total red and orange vegetables

8. Tomatoes

9. Other red and orange vegetables (excludes tomatoes)

10. Total starchy vegetables

11. Potatoes (white potatoes)

12. Other starchy vegetables (excludes white potatoes)

13. Other vegetables

14. Beans and peas computed as vegetables

15. Total grains

16. Whole grains

17. Refined grains

18. Total protein foods

19. Total meat, poultry, and seafood

20. Meat (beef, veal, pork, lamb, game)

21. Cured meat (frankfurters, sausage, corned beef, cured ham and luncheon meat made from beef, pork, poultry)

22. Organ meat (from beef, veal, pork, lamb, game, poultry)

23. Poultry (chicken, turkey, other fowl)

24. Seafood high in $n-3$ fatty acids

25. Seafood low in n-3 fatty acids

26. Eggs

27. Soybean products (excludes calcium fortified soy milk and mature soybeans)

28. Nuts and seeds

29. Beans and peas computed as protein foods

30. Total dairy (milk, yogurt, cheese, whey)

31. Milk (includes calcium fortified soy milk)

32. Yogurt

33. Cheese

34. Oils

35. Solid fats

36. Added sugars

37. Alcoholic drinks

From USDA-ARS (2014). 


\section{Appendix $\mathrm{H}$ \\ Modified SAS Code for the NCI Population Ratio Method to Obtain Average HEI Total Scores for Adults aged 60+}

(SAS code was created by the $\mathrm{NCl}$ as a tool for researchers, modifications are the green annotations)

The NCI SAS code is available at http://epi.grants.cancer.gov/hei/tools.html

libname demo1 xport "C:IUsers|MochalDocuments|DEMO_F.xpt"; libname demo2 xport "C:IUsers|MochalDocuments|DEMO G.xpt"; libname fped "C:IUsers|MochalDocumentsIFPED"; libname tot1 xport "C:IUsers|MochalDocumentsIDR1TOT_F.xpt"; libname tot2 xport "C:IUsers|MochalDocumentsIDR1TOT_G.xpt";

\%include "C:IUsers\MochalDocuments|FPEDIhei2010.beanspeas.allocation.macro.sas"; \%include "C:IUsers\MochalDocuments\FPEDIhei2010.score.macro.sas";

*Get Food Patterns Equivalents for foods in NHANES 2009-2010 and 2011-2012;

data food;

merge fped.fped_dr1iff_0910

fped.fped_dr1iff_1112; *Merging the two 2-year datasets into one 4-year dataset;

by seqn;

if DR1DRSTZ=1;

if DRDINT=2

WHOLEFRUIT $=$ DR1I_F_CITMLB + DR1I_F_other; *Adding citrus

Fruits and melons + other fruits for one wholefruit variable;

DIET4YR=1/2*WTDRD1; *Calculating 4-year dietary sample weight;

run;

*Getting total nutrient intakes for foods in NHANES 2009-2010;

data nutrient (keep=seqn wtdrd1 dr1tkcal dr1tcarb dr1ttfat dr1talco

dr1tsodi dr1drstz dr1tmfat dr1tpfat dr1tsfat drdint); set tot1.DR1TOT_F;

if DR1DRSTZ=1;

if DRDINT=2;

run;

${ }^{*}$ Getting total nutrient intakes for foods in NHANES 2011-2012;

data nutrient2 (keep=seqn WTDRD1 dr1tkcal dr1tcarb dr1ttfat dr1talco dr1tsodi dr1drstz dr1tmfat dr1tpfat dr1 tsfat drdint); set tot2.DR1TOT_G;

if DR1DRSTZ=1;

if DRDINT=2;

run; 
proc sort data=nutrient;

by seqn;

run;

proc sort data=nutrient2;

by seqn;

run;

*Merging total nutrient intakes for foods in NHANES 2009-2010 and 2011-2012 for a 4year dataset;

data nutrientboth;

merge nutrient nutrient2;

by seqn;

DIET4YR=1/2*WTDRD1;

run;

*Getting demographic variables from NHANES 2009-2010;

data demo (keep=seqn ridageyr riagendr sddsrvyr sdmvpsu sdmvstra dmdmartl dmahhsiz indfmpir); set demo1.demo_f;

run;

proc sort data=demo;

by seqn;

run;

*Getting demographic variables from NHANES 2009-2010;

data demo2 (keep=seqn ridageyr riagendr sddsrvyr sdmvpsu sdmvstra dmdmartl dmdhhsiz indfmpir); set demo2.demo_g;

run;

proc sort data=demo2;

by seqn;

run;

*Merging demographic variables in NHANES 2009-2010 and 2011-2012 for a 4-year dataset;

data demoboth; merge demo demo2;

by seqn;

run;

*Merging the demographic and food intakes 4-year datasets;

data cohort; 
merge nutrientboth demoboth;

by seqn;

run;

data cohort;

set cohort;

if DR1DRSTZ=1;

if RIDAGEYR >=60; *Keeping only participants aged 60+;

run;

proc sort data $=$ food;

by seqn;

run;

proc means data $=$ food noprint;

by seqn;

var DR1I_F_CITMLB -- DR1I_A_DRINKS wholefruit;

output out=pyrcalc sum $=$;

run;

data both;

merge cohort (in=c) nutrientboth (in=f) pyrcalc (in=P) demoboth;

by seqn;

if $\mathrm{c}$;

run;

data both;

set both;

by seqn;

array pyrvar DR1I_F_CITMLB -- DR1I_A_DRINKS wholefruit;

do over pyrvar;

if pyrvar $<0$ then pyrvar=0;

end;

run;

data both;

set both;

by seqn;

MONOPOLY $=$ dr1 tmfat + dr1tpfat;

ALLMEAT = DR1I_PF_MEAT + DR1I_PF_CUREDMEAT + DR1I_PF_ORGAN + DR1I_PF_PŌULT + DR1I_PF_ĒGGS + DR1I_PF_NUTSDS + DR1I_PF_SOY;

SEAPLANT = DR1I_PF_SEAFD_HI + DR1I_PF_SEAFD_LOW + DR1I_PF_SOY

$+$

DR1I_PF_NUTSDS;

ADDSUGC $=16^{\bar{*}} \mathrm{DR} \overline{1} \mathrm{I} \_A D D \_S U G A R S$;

SOLFATC=DR1I_SOLID_FATS*9;

MAXALCGR $=13^{*}(\mathrm{dr} 1 \mathrm{tkcal} / 1000)$;

if dr1TALCO <= MAXALCGR then EXALCCAL=0;

else if dr1TALCO > MAXALCGR then EXALCCAL=7*(dr1TALCO-

MAXALCGR); 
EMPTYCAL10=ADDSUGC+SOLFATC+EXALCCAL;

run;

data one;

set both;

array comp (15) dr1TKCAL DR1I_v_total DR1I_v_drkgr DR1I_v_legumes

DR1I_f_total wholefruit DR1I_g_whole DR1I_d_total

allmeat seaplant monopoly dr1tsfat dr1tsodi

do $\mathrm{i}=1$ to 15 ;

DR1I_g_refined emptycal10;

$\mathrm{VBL}=\operatorname{comp}(\mathrm{i})$;

dum_num = i;

output;

end;

run;

data one;

set one;

array comp (15) dr1TKCAL DR1I_v_total DR1I_v_drkgr DR1I_v_legumes

DR1I_f_total wholefruit DR 1 1__g_whole

DR1I_d_total allmeat seaplant monopoly dr1tsfat

do $i=1$ to 15 ;

dr1tsodi DR1I_g_refined emptycal10;

if dum_num $=\mathrm{i}$ then $\operatorname{comp}(\mathrm{i})=1$;

else comp(i) = 0;

end;

drop i dum_num;

run;

proc surveyreg data=one;

strata SDMVSTRA;

cluster SDMVPSU;

weight DIET4YR;

model VBL=dr1TKCAL DR1I_v_total DR1I_v_drkgr DR1I_v_legumes

DR1I_f_total wholefruit DR1I_g_whöle DR1I_d_total allmeat seaplant

monopoly dr1tsfat dr1tsodi DR1I_g_refined emptycal10/noint covb;

ods output covb=csd_cov;

title2 "Tricking surveyreg into giving us the covariance matrix of means";

run;

proc print data $=$ csd_cov;

title2 "Printout of csd_cov dataset-uses complex survey info";

run;

proc means data=both $\mathrm{n}$ min max mean;

weight DIET4YR;

var dr1TKCAL DR1I_v_total DR1I_v_drkgr DR1I_v_legumes

DR1I_f_total wholefruit DR1I_g_whole DR1I_d_total allmeat seaplant

monopoly dr1tsfat dr1tsodi DR1I_g_refined emptycal10;

title2 "look at weighted means"; 
output out=wtdm mean= ;

run;

data covdata (drop=parameter);

set csd_cov;

TYPE_="COV ";

_NAME_=Parameter;

run;

data wtdm (drop=_TYPE__FREQ_);

set wtdm;

run;

data wtdm;

set wtdm;

_TYPE_="MEAN";

run;

data covdata;

set covdata wtdm;

run;

proc print data $=$ covdata;

title2 "input to simnorml";

run;

proc simnormal data $=$ covdata $($ type $=$ cov $)$ numreal $=10000$ seed $=51230077$

outseed out=sim_data;

var dr1TKCAL DR1I_v_total DR1I_v_drkgr DR1I_v_legumes

DR1I_f_total wholefruit DR1I_g_whole DR1I_d_total allmeat seaplant monopoly dr1tsfat dr1tsodi DR1I_g_refined

run; emptycal10;

proc means data $=$ sim_data $\mathrm{n}$ nmiss min max mean stddev; var dr1TKCAL DR1I_v_total DR1I_v_drkgr DR1I_v_legumes

DR1I_f_total wholefruit DR1I_g_whole DR1I_d_total allmeat seaplant monopoly dr1tsfat dr1tsodi DR1I_g_refined emptycal10;

title2 "distributions of simulated data";

run;

proc print data $=$ sim_data (obs $=20$ );

title2 "listing of 20 records from simulated data";

run;

*THE LEGUME ALLOCATION MACRO;

\%LEG2010A (indat=sim_data,

kcal=dr1TKCAL,

allmeat=allmeat, 


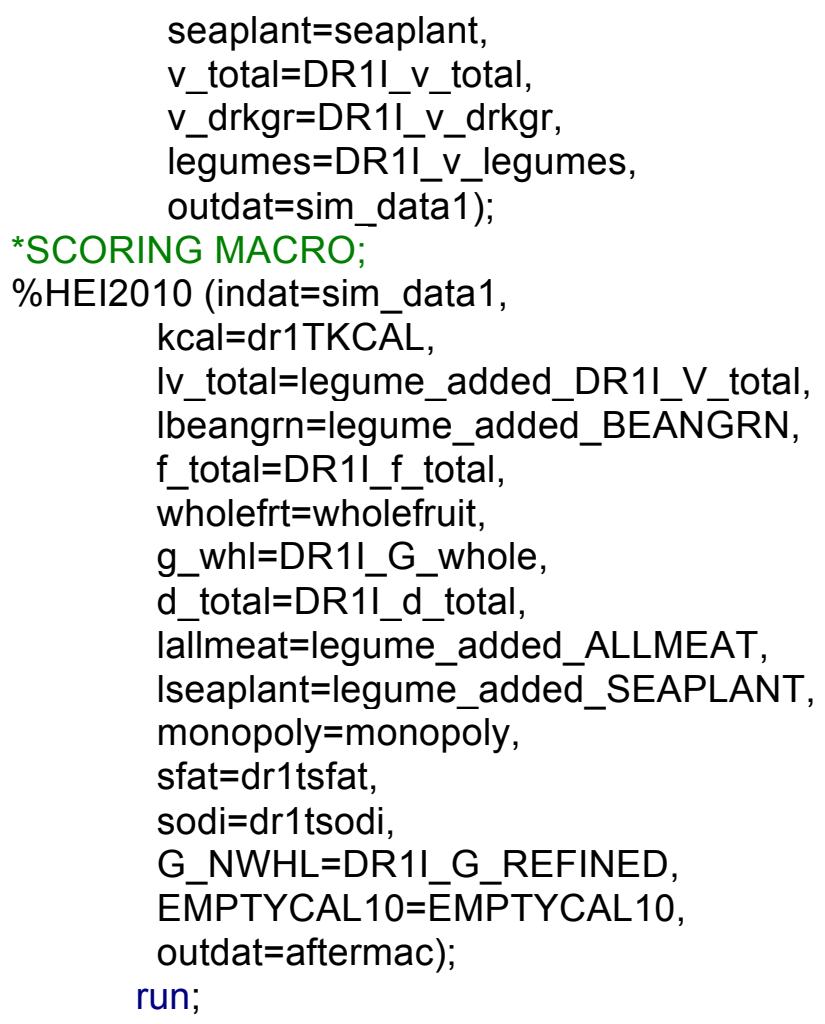

proc means data=aftermac $\mathrm{n}$ nmiss min max mean stddev;

var legume_added_DR1I_v_total legume_added_beangrn

legume_added_allmeat legume_added_seaplant vegden grbnden

frtden whfrden wgrnden dairyden meatden seaplden faratio

sodden rgden sofa_perc;

title2 "after legume allocation and hei 2010 scoring macro";

run;

proc univariate data=aftermac noprint;

var HEIX1_TOTALVEG HEIX2_GREEN_AND_BEAN HEIX3_TOTALFRUIT

HEIX4_WHOLEFRUIT HEIX5_WHOLEGRAIN HEIX6_TOTALDAIRY

HEIX7_TOTPROT HEIX8_SEAPLANT_PROT HEIX9_FATTYACID

HEIX10_SODIUM HEIX11_REFINEDGRAIN HEIX12_SOFAAS

HEI2010_TOTAL_SCORE;

output out $=$ ci pctlpts $=2.597 .5$ pctlpre $=\mathrm{h} 1$ 1_h2_h3_h4_h5_h6_

run;

$$
\text { h7_h8_h9_h10_h11_h12_totscore_; }
$$

proc means data=aftermac noprint;

var HEIX1_TOTALVEG HEIX2_GREEN_AND_BEAN HEIX3_TOTALFRUIT HEIX4_WHOLEFRUIT HEIX5_WHOLEGRAIN HEIX6_TOTALDAIRY

HEIX7_TOTPROT HEIX8_SEAPLANT_PROT HEIX9_FATTYACID

HEIX10_SODIUM HEIX11__REFINEDGRAIN HEIX12_SOFAAS HEI2010_TOTAL_SCORE;

output out=stat min=h1_min h2_min h3_min h4_min h5_min h6_min h7_min h8_min h9_min h̄10_min h11_min h12_min totscore_min 
max=h1_max h2_max h3_max h4_max h5_max h6_max h7_max h8_max h9_max h10_max h11_max h12_max totscore_max

mean $=\mathrm{h} 1$ _mean $\mathrm{h} \overline{2}$ _mean $\mathrm{h} \overline{3}$ mean $\mathrm{h} \overline{4}$ mean $\mathrm{h} 5$ mean $\mathrm{h} 6$ mean $\mathrm{h} 7$ _mean h8_mean h9_mean h10_mean h 11 _mean $\bar{h} 12$ mean totscore_mean

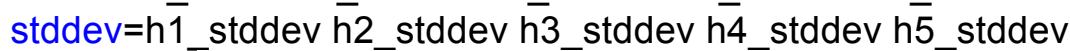
h6_stddev h7_stddev h8_stddev h9_stddev h10_stddev h11_stddev h12_stddev totscore_stddev;

run;

data all;

merge ci stat;

run;

data result (keep=score slabel min max mean stderr low high);

set all;

score="HElx1 ";

slabel="HEI-2010 Component 1 Total Vegetables";

$\min =\mathrm{h} 1$ min;

$\max =\mathrm{h} 1$ max;

mean $=\mathrm{h} 1$ mean;

stderr=h1 stddev;

low $=$ h1 2 5;

high=h1_ 97 ;

output result;

score="HEIx2";

slabel="HEI-2010 Component 2 Greens and Beans";

$\min =\mathrm{h} 2 \mathrm{~min}$;

$\max =\mathrm{h} 2$ max;

mean $=\mathrm{h} \overline{2}$ mean;

stderr=h2_stddev;

low $=\mathrm{h} 2 \_2 \_5$;

high=h2_97_5;

output result;

score="HEIx3";

slabel="HEI-2010 Component 3 Total Fruit";

min=h3_min;

$\max =\mathrm{h} 3$ max;

mean $=h \overline{3}$ _mean;

stderr=h3_stddev;

low=h3_2_5;

high=h3_97_5;

output result;

score="HElx4";

slabel="HEI-2010 Component 4 Whole Fruit";

min=h4_min;

$\max =\mathrm{h} 4$ max;

mean $=\mathrm{h} 4$ _mean;

stderr=h4_stddev;

low=h4_2_5;

high $=\mathrm{h} 4 \quad 97 \quad 5$;

output result; 


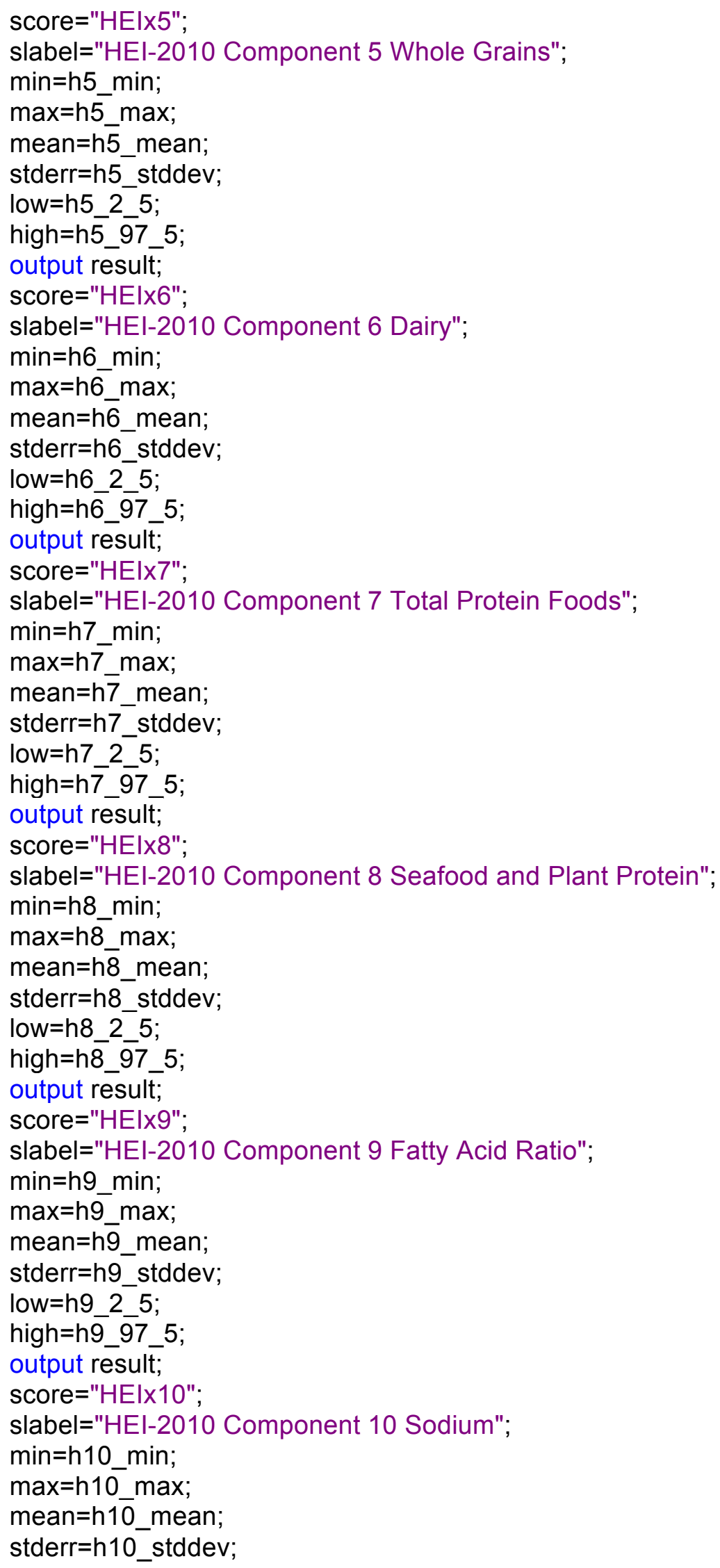




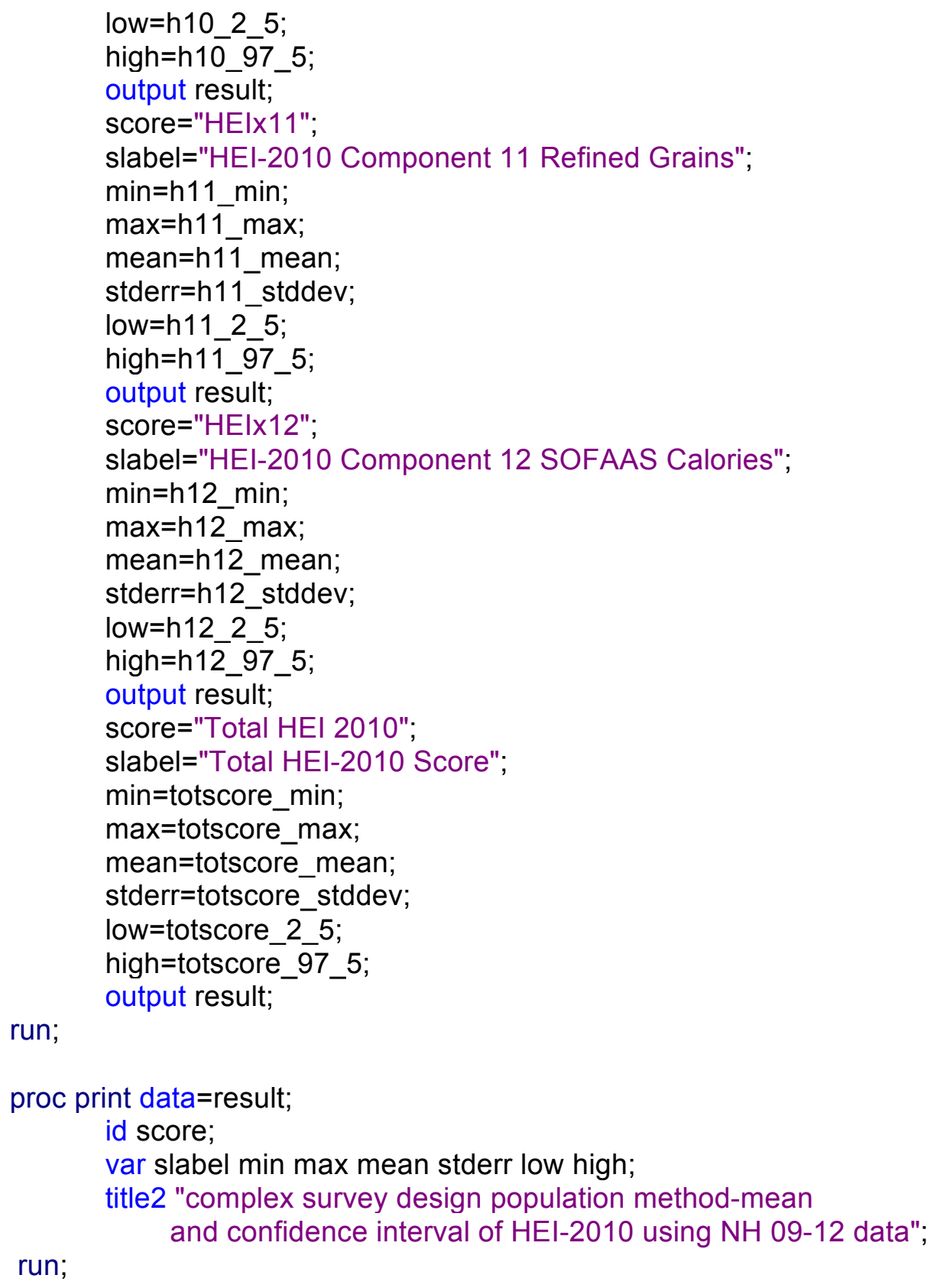




\section{Appendix I}

Modified SAS Code to Obtain Individual Average HEI Total Scores for Adults aged 60+

(SAS code was created by the $\mathrm{NCl}$ as a tool for researchers, modifications are the green annotations)

The NCI SAS code is available at http://epi.grants.cancer.gov/hei/tools.html

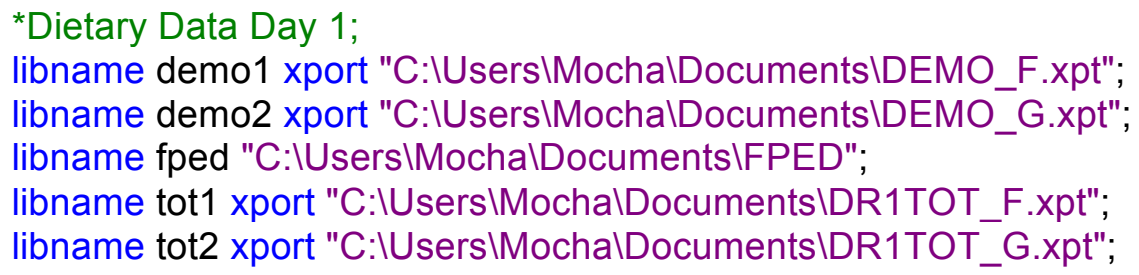

*Getting total nutrient intakes for foods in NHANES 2009-2010;

data nutrient (keep=seqn WTDRD1 WTDR2D dr1tkcal dr1tcarb dr1ttfat dr1talco dr1 tsodi dr1drstz dr1tmfat dr1tpfat dr1tsfat drdint);

set tot1.DR1TOT_F;

if drdint=2;

if $\mathrm{dr} 1 \mathrm{drstz}=1$;

run;

*Getting total nutrient intakes for foods in NHANES 2011-2012;

data nutrient2 (keep=seqn WTDRD1 WTDR2D dr1tkcal dr1tcarb dr1ttfat dr1talco dr1tsodi dr1drstz dr1tmfat dr1tpfat dr1tsfat drdint);

set tot2.DR1TOT_G;

if drdint=2; 


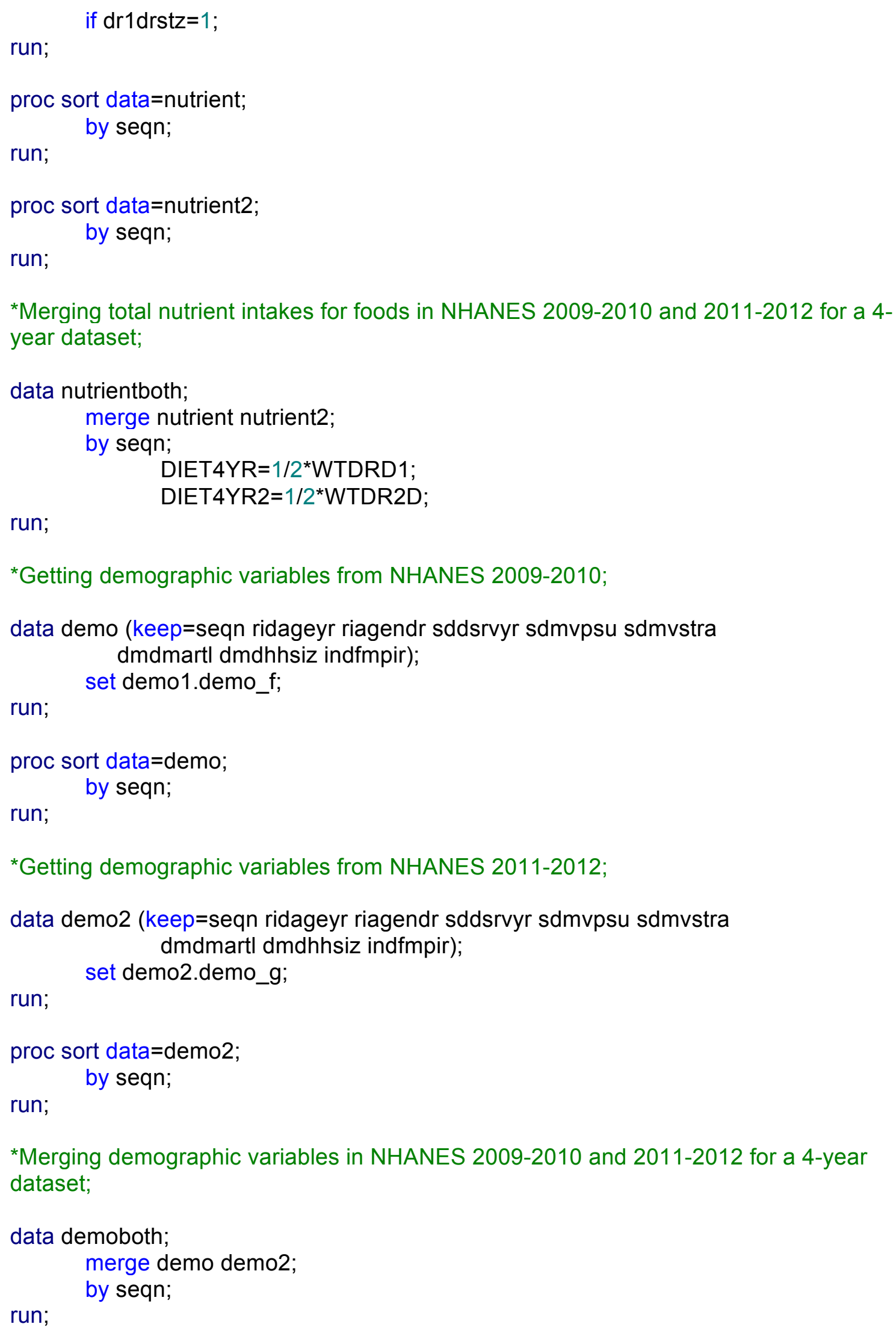


*Merging the demographic and food intakes 4-year datasets;

data cohort;

merge nutrientboth demoboth;

by seqn;

run;

data cohort;

set cohort;

if DR1DRSTZ=1;

if RIDAGEYR >=60; *Keeping only participants aged 60+;

run;

proc means data=food noprint;

by seqn;

var DR1I_F_CITMLB -- DR1I_A_DRINKS wholefruit;

output out=pyrcalc sum $=$;

run;

data both;

merge cohort (in $=c$ ) nutrientboth (in=f) pyrcalc (in=P) demoboth;

by seqn;

if $\mathrm{c}$;

run;

data both;

set both;

by seqn;

array pyrvar DR1I_F_CITMLB -- DR1I_A_DRINKS wholefruit;

do over pyrvar;

if pyrvar $<0$ then pyrvar=0;

end;

run;

data both;

set both;

by seqn;

MONOPOLY=dr1tmfat + dr1tpfat;

ALLMEAT = DR1I_PF_MEAT + DR1I_PF_CUREDMEAT + DR1I_PF_ORGAN + DR1I_PF_PŌULT + DR1I_PF_ĒGGS + DR1I_PF_NUTSDS + DR1I_PF_SOY;

SEAPLANT $=$ DR1I_PF_SEAFD_HI + DR1I_PF_SEAFD_LOW + DR1I_PF_SOY

$+$

DR1I_PF_NUTSDS;

ADDSUGC $=16^{\star}$ DR 11 ADD_SUGARS;

SOLFATC=DR1I_SOLID_FATS*9;

MAXALCGR $=13^{*}(\mathrm{dr} 1 \mathrm{tkcal} / 1000)$;

if dr1TALCO <= MAXALCGR then EXALCCAL=0;

else if dr1TALCO > MAXALCGR then EXALCCAL=7*(dr1TALCO-

MAXALCGR);

EMPTYCAL10=ADDSUGC+SOLFATC+EXALCCAL; 
run;

*The legume allocation macro code;

data both;

set both;

by seqn;

MBMAX $=2.5^{*}(\mathrm{DR} 1 \mathrm{TKCAL} / 1000)$;

if ALLMEAT < MBMAX then do;

MEATLEG=DR1I_V_legumes*4;

NEEDMEAT $=$ MBMAX-ALLMEAT;

if MEATLEG <= NEEDMEAT THEN DO;

LEGTYPE='ALLMEAT';

legume_added_ALLMEAT=ALLMEAT+MEATLEG;

legume_added_SEAPLANT=SEAPLANT+MEATLEG;

legume_added_DR1I_V_TOTAL=DR1I_V_TOTAL;

legume_added_BEANGRN=DR1I_V_DRKGR;

END;

ELSE IF MEATLEG > NEEDMEAT THEN DO;

LEGTYPE='MEAT/VEG';

EXTRMEAT=MEATLEG-NEEDMEAT;

EXTRLEG=EXTRMEAT/4;

legume_added_ALLMEAT=ALLMEAT+NEEDMEAT;

legume added_SEAPLANT=SEAPLANT+NEEDMEAT;

legume_added_DR1I_V_TOTAL=DR1I_V_TOTAL+EXTRLEG;

legume_added_BEANG $\bar{R} N=D R 1$ 1_V_DRKGR+EXTRLEG;

END;

END;

ELSE IF ALLMEAT >= MBMAX THEN DO;

LEGTYPE='ALLVEG';

legume_added_ALLMEAT=ALLMEAT;

legume_added_SEAPLANT=SEAPLANT;

legume_added_DR1I_V_TOTAL=DR1I_V_TOTAL+DR1I_V_LEGUMES;

legume_added_BEAN̄G $\bar{R} N=D R 1 I$ V_DRKGR+DR1I_V_LEGUMES;

END;

run;

*The HEI-2010 Scoring Macro Code to get HEI component scores per individual;

*Legume added scores;

data both;

set both;

by seqn;

if DR1TKCAL >0 then

vegstan=legume_added_DR1I_V_TOTAL/(DR1Tkcal/1000);

TOTALVEGHEI=5*(vegstan/1.1);

if TOTALVEGHEI >5 then TOTALVEGHEI=5;

if vegstan $=0$ then TOTALVEGHEI=0;

if DR1TKCAL >0 then

grnbnstan=legume_added_BEANGRN/(DR1TKCAL/1000);

GREENBEANHEI=5*(grnbnstan/0.2); 
if GREENBEANHEI >5 then GREENBEANHEI=5;

if grnbnstan=0 then GREENBEANHEI=0;

if DR1TKCAL >0 then

meatstan=legume_added_ALLMEAT/(DR1TKCAL/1000);

TOTPROTHEI $=5^{*}($ meatstan/2.5);

if TOTPROTHEI > 5 then TOTPROTHEI=5;

if meatstan $=0$ then TOTPROTHEI=0;

if DR1TKCAL >0 then

seastan=legume_added_SEAPLANT/(DR1TKCAL/1000);

SEAPLANTHEI $=5^{*}$ (seastan $\left./ 0.8\right)$;

if SEAPLANTHEI $>5$ then SEAPLANTHEI=5;

if seastan=0 then SEAPLANTHEI=0;

run;

*Total Fruit;

data both;

set both;

by seqn;

if DR1TKCAL >0 then fruitstan=DR1I_F_TOTAL/(DR1TKCAL/1000);

TOTALFRUITHEI $=5$ (fruitstan/0.8);

if TOTALFRUITHEI >5 then TOTALFRUITHEI=5;

if fruitstan=0 then TOTALFRUITHEI=0;

run;

*Whole fruit;

data both;

set both;

by seqn;

if DR1TKCAL > 0 then wholestan=wholefruit/(DR1TKCAL/1000);

WHOLEFRUITHEI $=5 *$ (wholestan/0.4);

if WHOLEFRUITHEI $>5$ then WHOLEFRUITHEI=5;

if wholestan=0 then WHOLEFRUITHEI=0;

run;

*Whole Grain;

data both;

set both;

by seqn;

if DR1TKCAL > 0 then grainstan=DR1I_G_whole/(DR1TKCAL/1000);

WHOLEGRAINHEI=10* (grainstan/1.5);

if WHOLEGRAINHEI >10 THEN WHOLEGRAINHEI=10;

if grainstan=0 then WHOLEGRAINHEI=0;

run;

${ }^{*}$ Refined grains;

data both; 


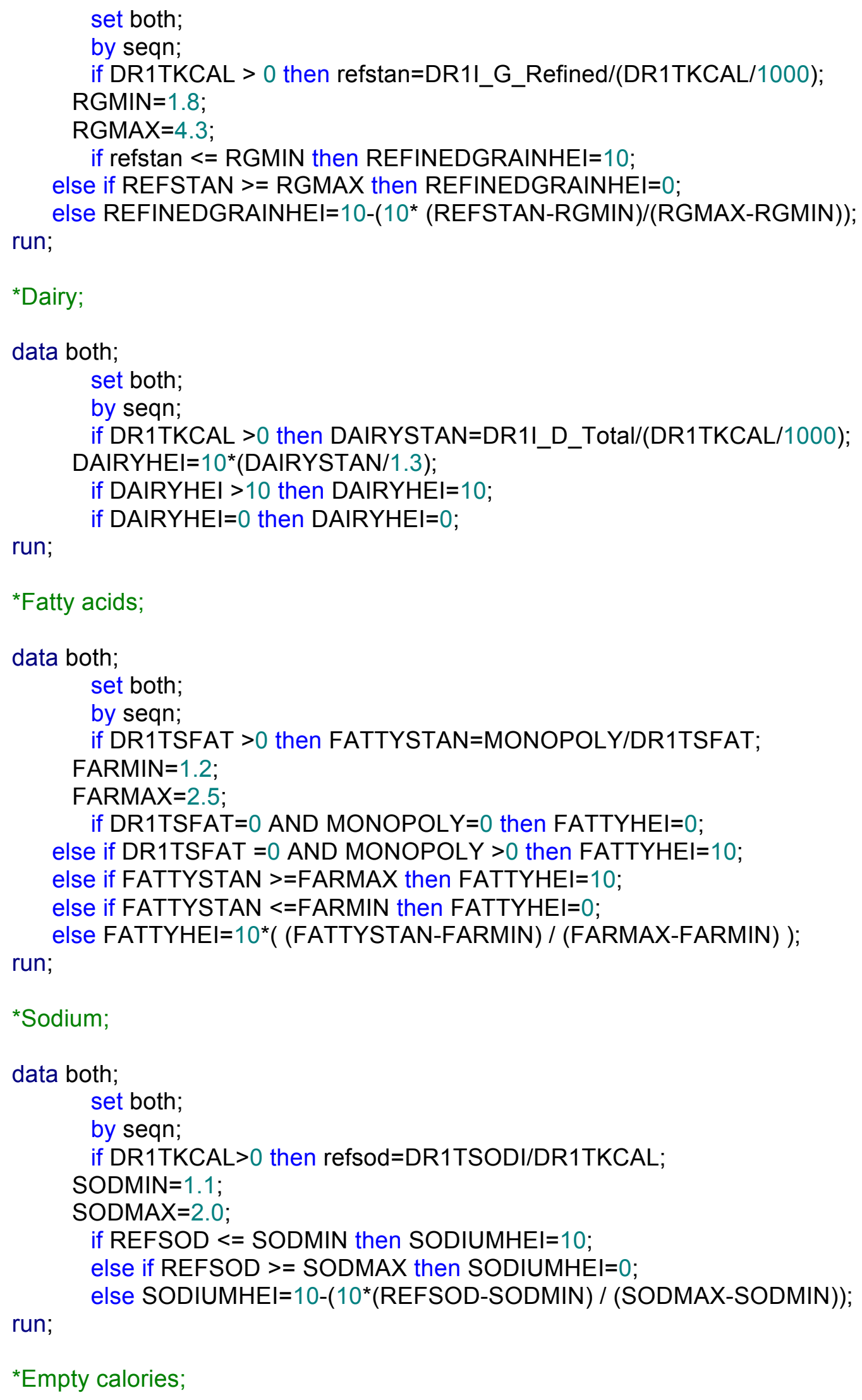




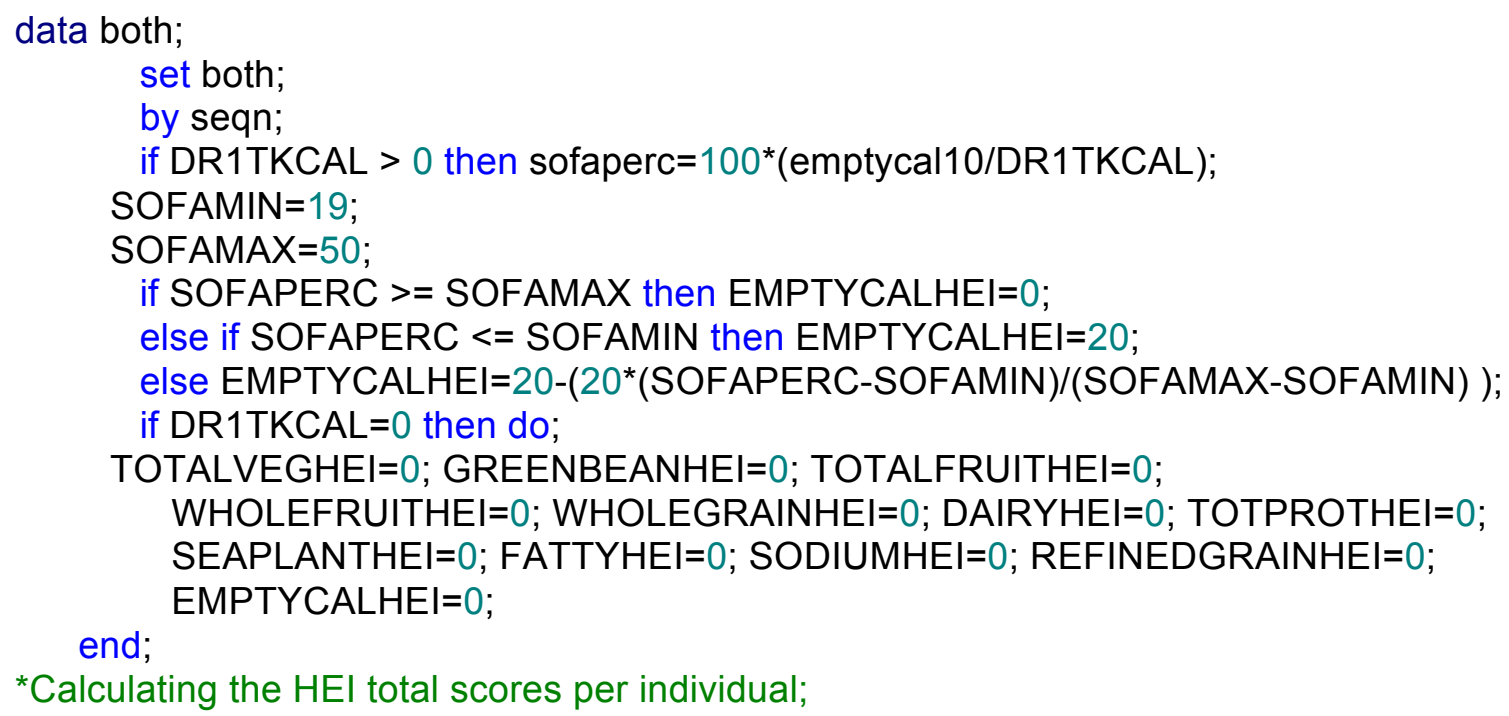

${ }^{*}$ Calculating the HEl total scores per individual;

HEITOTALSCORE=TOTALVEGHEI+GREENBEANHEI+TOTALFRUITHEI+WHOLEFR UITHEI+WHOLEGRAINHEI+DAIRYHEI+TOTPROTHEI+SEAPLANTHEI+FATTYHEI+S ODIUMHEI+REFINEDGRAINHEI+EMPTYCALHEI;

run; 